September, 2004

hep-th/0409191

HUTP-03/A053

RUNHETC-2003-26

\title{
$M$ theory and Singularities of Exceptional Holonomy Manifolds
}

\author{
Bobby S. Acharya ${ }^{1}$ and Sergei Gukov ${ }^{2}$ \\ ${ }^{1}$ Abdus Salam International Centre for Theoretical Physics, \\ Strada Costiera 11, 34100 Trieste, Italy. \\ bacharya@ictp.trieste.it \\ ${ }^{2}$ Jefferson Physical Laboratory, Harvard University, \\ Cambridge, MA 02138, U.S.A. \\ gukov@schwinger . harvard.edu
}

\begin{abstract}
$M$ theory compactifications on $G_{2}$ holonomy manifolds, whilst supersymmetric, require singularities in order to obtain non-Abelian gauge groups, chiral fermions and other properties necessary for a realistic model of particle physics. We review recent progress in understanding the physics of such singularities. Our main aim is to describe the techniques which have been used to develop our understanding of $M$ theory physics near these singularities. In parallel, we also describe similar sorts of singularities in $\operatorname{Spin}(7)$ holonomy manifolds which correspond to the properties of three dimensional field theories. As an application, we review how various aspects of strongly coupled gauge theories, such as confinement, mass gap and non-perturbative phase transitions may be given a simple explanation in $M$ theory.
\end{abstract}




\section{Contents}

1 Introduction $\quad 2$

2 Riemannian Manifolds of Special Holonomy $\quad 6$

2.1 Holonomy Groups . . . . . . . . . . . . . . . . . . . . . . . . . 6

2.2 Relation Between Holonomy and Supersymmetry . . . . . . . . . . . . 8

2.3 Invariant Forms and Minimal Submanifolds . . . . . . . . . . . . . . . 10

2.4 Why Exceptional Holonomy is Hard . . . . . . . . . . . . . . . . . . . . 14

3 Construction of Manifolds With Exceptional Holonomy 16

3.1 Compact Manifolds . . . . . . . . . . . . . . . . . . . . . . . 16

3.2 Non-compact Manifolds . . . . . . . . . . . . . . . . . . . . . . . . . . 20

$4 M$ theory on Smooth Special Holonomy Manifolds 28

$5 \quad M$ theory Dynamics on Singular Special Holonomy Manifolds 33

5.1 Low Energy Dynamics via Duality with the Heterotic String . . . . . . 34

5.2 Low Energy Dynamics via IIA Duals . . . . . . . . . . . . . . . 44

6 Chiral Fermions from Conical Singularities in $G_{2}$-manifolds $\quad 51$

6.1 Hints from Anomaly-Inflow. . . . . . . . . . . . . . . . . . . . . 51

6.2 Chiral Fermions via Duality With The Heterotic String . . . . . . . . . 53

6.3 Other Gauge Groups and Matter Representations . . . . . . . . . . . . 60

7 Topology Change in $M$ theory on Exceptional Holonomy Manifolds 63

7.1 Topology Change in $M$ theory . . . . . . . . . . . . . . . . . . 63

7.2 Relation to Geometric Transition . . . . . . . . . . . . . . . . . . . 69

8 Quantum Super-Yang-Mills from $G_{2}$ Manifolds $\quad 75$

8.1 Super Yang-Mills Theory. . . . . . . . . . . . . . . . . . 75

8.2 Theta angle and Coupling Constant in M theory. . . . . . . . . . . 77

8.3 Superpotential in M theory. . . . . . . . . . . . . . . . 77

8.4 M theory Physics on ADE-singular $G_{2}$-manifolds. . . . . . . . . . . . 84

8.5 Confinement from $G_{2}$-manifolds. . . . . . . . . . . . . . . 85

8.6 Mass Gap from $G_{2}$-manifolds. . . . . . . . . . . . . . . . . . . . 86 


\section{Introduction}

$M$ theory is a promising candidate for a consistent quantum theory of gravity. The theory unifies all of the five consistent superstring theories. $M$ theory is locally supersymmetric and at long distances describes physics in spacetimes with eleven dimensions. The traditional approach to obtaining large four dimensional universes from theories with more than four dimensions is to assume that the "extra dimensions" are small. At energies below the compactification scale of the extra dimensions, the physics is four dimensional and the detailed properties of that physics is determined by the properties of the metric of the extra dimensions.

Recently, $M$ theory compactifications on manifolds with exceptional holonomy have attracted considerable attention. The main motivation to study such models is that they have all the ingredients required to embed phenomenologically interesting quantum field theories with minimal supersymmetry into a unified theory containing gravity.

Perhaps the most intriguing reason that makes supersymmetry one of our best candidates for physics beyond the Standard Model is the unification of gauge couplings and a possible mechanism for understanding the large hierarchy in scale between the masses of particles at the electroweak scale and the much higher unification scale

$$
M_{G U T} \approx 10^{16} \mathrm{GeV}
$$

The main idea of Grand Unification is that three out of four fundamental forces in nature (strong, weak, and electro-magnetic) combine into a single force at high energies. At low energies all of these forces are mediated by an exchange of gauge fields and to very high accuracy can be described by the Standard Model of fundamental interactions with the gauge group

$$
U_{Y}(1) \otimes S U_{L}(2) \otimes S U_{c}(3)
$$

Even though the coupling constants, $\alpha_{i}$, associated with these interactions are dimensionless, in quantum field theory they become functions of the energy scale $\mu$. In particular, using the experimental data from the Large Electron Positron accelerator (LEPEWWG) and from Tevatron for the values of $\alpha_{i}$ at the electroweak scale [1], one can predict the values of $\alpha_{i}(\mu)$ at arbitrary energy scale, using quantum field theory. Then, if one plots all $\alpha_{i}(\mu)$ as functions of $\mu$ on the same graph, one finds that near the Planck scale three curves come close to each other, but do not meet at one point. The latter observation means that the unification can only be achieved if new physics enters between the electroweak and the Planck scale, so that at some point all coupling constants can be made equal,

$$
\alpha_{1}\left(M_{G U T}\right)=\alpha_{2}\left(M_{G U T}\right)=\alpha_{3}\left(M_{G U T}\right)
$$


When this happens, the three gauge interactions have the same strength and can be ascribed a common origin.

An elegant and simple solution to the unification problem is supersymmetry, which leads to a softening of the short distance singularities and, therefore, modifies the evolution of coupling constants. In fact, if we consider a minimal supersymmetric generalization of the Standard Model (MSSM) where all the superpartners of the known elementary particles have masses above the effective supersymmetry scale

$$
M_{S U S Y} \approx 1 \mathrm{TeV}
$$

then a perfect unification (1.3) can be obtained at the GUT scale (1.1) [2].

Moreover, supersymmetry might naturally explain the large difference between the unification scale $M_{G U T}$ and the electroweak scale $\left(m_{E W} \approx 100 \mathrm{GeV}\right)$ called the "hierarchy problem". Even if some kind of fine tuning in a GUT theory can lead to a very small number $m_{E W} / M_{G U T} \sim 10^{-14}$, the problem is to preserve the hierarchy after properly accounting for quantum corrections. For example, the one-loop correction to the Higgs mass $m_{H}$ in a non-supersymmetric theory is quadratically divergent, hence $\delta m_{H}^{2} \sim \Lambda^{2}$. This is too large if the cutoff scale $\Lambda$ is large. Clearly, such quantum corrections destroy the hierarchy, unless there is a mechanism to cancel these quadratic divergences. Again, supersymmetry comes to the rescue. In supersymmetric quantum field theory all quadratic corrections automatically cancel to all orders in perturbation theory due to opposite contributions from bosonic and fermionic fields.

The unification of gauge couplings and a possible solution of the hierarchy problem demonstrate some of the remarkable properties of supersymmetry. Namely, the dynamics of supersymmetric field theories is usually rather constrained (an example of this is the cancellation of ultraviolet divergences that was mentioned above), and yet rich enough to exhibit many interesting phenomena, such as confinement, Seiberg duality, non-perturbative phase transitions, etc. It turns out that many of these phenomena can receive a relatively simple and elegant explanation in the context of $M$ theory and its string theory approximations.

In $M$ theory, there are several natural looking ways to obtain four large spacetime dimensions with minimal $(\mathcal{N}=1)$ supersymmetry from compactification on a manifold $X$ with special holonomy. The most well studied possibility is the heterotic string theory on a Calabi-Yau space $X$ [3]. A second way to obtain vacua with $\mathcal{N}=1$ supersymmetry came into focus with the discovery of string dualities, which allow definite statements to be made even in the regions where perturbation theory can not be used [4]. It consists of taking $X$ to be an elliptically fibered Calabi-Yau four-fold as a background in $\mathrm{F}$ theory. These examples are all limits of $M$ theory on $X$. The third possibility, which will be one of the main focal points of this review is to take 
$M$ theory on a 7 -manifold $X$ of $G_{2}$ holonomy. A central point concerning such $G_{2}$ compactifications is that, if $X$ is smooth, the four dimensional physics contains at most an abelian gauge group and no light charged particles. In fact, as we will see, in order to obtain more interesting four dimensional physics, $X$ should possess very particular kinds of singularity. At these singularities, extra light charged degrees of freedom are to be found.

Many of these compactifications are related by various string dualities that we will exploit below in order to study the dynamics of $M$ theory on singular manifolds of $G_{2}$ holonomy. An example of such a duality — which may be also of interest to mathematicians, especially those with an interest in mirror symmetry - is a duality between $M$ theory on $K 3$-fibered $G_{2}$-manifolds and the heterotic string theory on $T^{3}$ fibered Calabi-Yau threefolds. On the string theory side the threefold is endowed with a Hermitian-Yang-Mills connection $A$ and chiral fermions emerge from zero modes of the Dirac operator twisted by $A$, whereas on the $M$ theory side this gets mapped to a statement about the singularities of $X$.

Within the past few years there has been a tremendous amount of progress in understanding $M$ theory physics near singularities in manifolds of exceptional holonomy. In particular we now understand at which kinds of singularities in $G_{2}$-manifolds the basic requisites of the Standard Model - non-Abelian gauge groups and chiral fermions - are to be found $[5,6,7,8,9,10]$. One purpose of this review is to explain how this picture was developed in detail. We mainly aim to equip the reader with techniques and refer the interested reader to $[11,12,13,14]$ for more detailed discussions of phenomenological applications. Among other things, we shall see how important properties of strongly coupled gauge theories such as confinement and the mass gap can receive a semi-classical description in $M$ theory on $G_{2}$-manifolds. Similarly, Spin $(7)$ manifolds expose additional aspects of $M$ theory, related to the interesting dynamics of minimally supersymmetric gauge theories in $2+1$ dimensions.

In order to make this review self-contained and pedagogical, in the next section we start with an introduction to special holonomy. In section 3, we review the construction of manifolds with exceptional holonomy. Then, in section 4 , we derive the basic properties of $M$ theory on such a manifold, in the limit when the manifold is large and smooth. Using string dualities, in section 5 we explain how one can understand many aspects of the physics when the compactification manifold has various kinds of singularity. These techniques are used in later sections to explain various phenomena in $M$ theory on singular manifolds with exceptional holonomy. In section 6 we describe in detail the singularities of $G_{2}$ manifolds which give rise to chiral fermions. In section 7 , we review topology changing transitions in manifolds with $G_{2}$ and $\operatorname{Spin}(7)$ holonomy, and their relation to the so-called geometric transition in string theory. Finally, in sec- 
tion 8, we shall see how interesting aspects of Yang-Mills theory, such as confinement and a mass gap, receive a very simple explanation within the context of $M$ theory on a $G_{2}$ manifold.

We emphasise that, whilst manifolds of special holonomy provide elegant models of supersymmetric particle physics and gravity, there is a very important gap in our understanding: how supersymmetry is broken and why the cosmological constant is so small? 


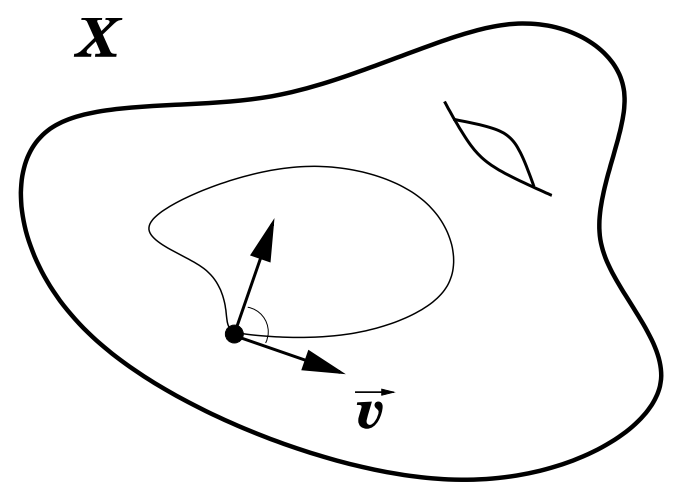

Figure 1: Parallel transport of a vector $\vec{v}$ along a closed path on the manifold $X$.

\section{Riemannian Manifolds of Special Holonomy}

\subsection{Holonomy Groups}

Consider an oriented manifold $X$ of real dimension $n$ and a vector $\vec{v}$ at some point on this manifold. One can explore the geometry of $X$ by parallel transporting $\vec{v}$ along a closed contractible path in $X$, see Figure 1. Under such an operation the vector $\vec{v}$ may not come back to itself. In fact, generically it will transform into a different vector that depends on the geometry of $X$, on the path, and on the connection which was used to transport $\vec{v}$. For a Riemannian manifold $X$ with metric $g(X)$, the natural connection is the Levi-Cevita connection. Furthermore, Riemannian geometry also tells us that the length of the vector covariantly transported along a closed path should be the same as the length of the original vector. But the direction may be different, and this is precisely what leads to the concept of holonomy.

The relative direction of the vector after parallel transport relative to that of the original vector $\vec{v}$ is described by holonomy. This is simply an $n \times n$ matrix, which on an $n$-dimensional, oriented manifold is an element of the special orthogonal group, $S O(n)$. It is not hard to see that the set of all holonomies themselves form a group, called the holonomy group, where the group structure is induced by the composition of paths and its inverse corresponds to a path traversed in the opposite direction. From the way we introduced the holonomy group, $\operatorname{Hol}(g(X))$, it seems to depend upon the choice of the base point. However, for generic choices of base points the holonomy group is in fact the same, and therefore $\operatorname{Hol}(g(X))$ becomes a true geometric characteristic of the space $X$ with metric $g(X)$. By definition, we have

$$
H o l(g(X)) \subseteq S O(n)
$$

where the equality holds for sufficiently generic metric on $X$. 


\begin{tabular}{|c|c|c|}
\hline Metric & Holonomy & Dimension \\
\hline \hline Kähler & $U\left(\frac{n}{2}\right)$ & $n=$ even \\
\hline Calabi-Yau & $S U\left(\frac{n}{2}\right)$ & $n=$ even \\
\hline HyperKähler & $S p\left(\frac{n}{4}\right)$ & $n=$ multiple of 4 \\
\hline Quaternionic & $S p\left(\frac{n}{4}\right) S p(1)$ & $n=$ multiple of 4 \\
\hline Exceptional & $G_{2}$ & 7 \\
\hline Exceptional & $\operatorname{Spin}(7)$ & 8 \\
\hline Exceptional & $\operatorname{Spin}(9)$ & 16 \\
\hline
\end{tabular}

Table 1: Berger's list of holonomy groups.

In some special instances, however, one finds that $\operatorname{Hol}(g(X))$ is a proper subgroup of $S O(n)$. In such cases, we say that $(X, g(X))$ is a special holonomy manifold or a manifold with restricted holonomy. These manifolds are in some sense distinguished, for they exhibit special geometric properties. As we explain later in this section, these properties are typically associated with the existence of non-degenerate (in some suitable sense) $p$-forms which are covariantly constant. Such $p$-forms also serve as calibrations, and are related to the subject of minimal varieties.

The possible choices for $\operatorname{Hol}(g(X)) \subset S O(n)$ are limited, and were classified by M. Berger in 1955 [15]. Specifically, for $(X, g(X))$ simply-connected and neither locally a product nor symmetric, the only possibilities for $\operatorname{Hol}(g(X))$, other than the generic case of $S O(n)$, are $U\left(\frac{n}{2}\right), S U\left(\frac{n}{2}\right), S p\left(\frac{n}{4}\right) \cdot S p(1), S p\left(\frac{n}{4}\right), G_{2}, \operatorname{Spin}(7)$ or $\operatorname{Spin}(9)$, see Table 1. The first four of these correspond, respectively, to Kähler, Calabi-Yau, Quaternionic Kähler or hyper-Kähler manifolds. The last three possibilities are the so-called exceptional cases, which occur only in dimensions 7, 8 and 16, respectively. The case of 16-manifolds with Spin(9) holonomy is in some sense trivial since the Riemannian metric on any such manifold is always symmetric [16]. Manifolds with $G_{2}$ or $\operatorname{Spin}(7)$ holonomy - which will be our main subject - are called exceptional holonomy manifolds, since they occur only in dimension seven or eight. Let us say a few more words about these cases, in particular, remind the definition and properties of $G_{2}$ and $\operatorname{Spin}(7)$ groups.

The 14-dimensional simple Lie group $G_{2}$ is precisely the automorphism group of the octonions, $\mathbb{O}$. It may be defined as the set of elements of $G L(7, \mathbb{R})$, which preserves the 
following 3 -form on $\mathbb{R}^{7}$,

$$
\Phi=\frac{1}{3 !} \psi_{i j k} d x_{i} \wedge d x_{j} \wedge d x_{k}
$$

where $x_{1}, \ldots, x_{7}$ are coordinates on $\mathbb{R}^{7}$, and $\psi_{i j k}$ are totally antisymmetric structure constants of the imaginary octonions,

$$
\sigma_{i} \sigma_{j}=-\delta_{i j}+\psi_{i j k} \sigma_{k}, \quad i, j, k=1, \ldots 7
$$

In a particular choice of basis the non-zero structure constants are given by

$$
\psi_{i j k}=+1, \quad(i j k)=\{(123),(147),(165),(246),(257),(354),(367)\}
$$

The 21-dimensional Lie group $\operatorname{Spin}(7)$ is usually defined as the double cover of $S O(7)$. However, by analogy with the above definition of $G_{2}$ group, it is convenient to define $\operatorname{Spin}(7)$ as a subgroup of $G L(8, \mathbb{R})$, which preserves the following 4 -form on $\mathbb{R}^{8}$,

$$
\begin{aligned}
\Omega= & e^{1234}+e^{1256}+e^{1278}+e^{1357}-e^{1368}-e^{1458}-e^{1467}- \\
& -e^{2358}-e^{2367}-e^{2457}+e^{2468}+e^{3456}+e^{3478}+e^{5678}
\end{aligned}
$$

where $e^{i j k l}=d x_{i} \wedge d x_{j} \wedge d x_{k} \wedge d x_{l}$ and $x_{1}, \ldots, x_{8}$ are coordinates on $\mathbb{R}^{8}$.

Finally, we note that by allowing $\pi_{1}(X)$ to be non-trivial one can obtain proper subgroups of the above list of groups as holonomy groups of $(X, g(X))$.

\subsection{Relation Between Holonomy and Supersymmetry}

Roughly speaking, one can think of the holonomy group as a geometric characteristic of the manifold that restricts the properties that $X$ has. Namely, as the holonomy group becomes smaller the more constrained the properties of $X$ become. Conversely, for manifolds with larger holonomy groups the geometry is less restricted.

This philosophy becomes especially helpful in the physical context of superstring/ $M$ theory compactifications on $X$. There, the holonomy of $X$ becomes related to the degree of supersymmetry preserved in compactification: manifolds with larger holonomy group preserve a smaller fraction of the supersymmetry. This provides a nice link between the 'geometric symmetry' (holonomy) and the 'physical symmetry' (supersymmetry). In Table 2 we illustrate this general pattern with a few important examples, which will be used later.

The first example in Table 2 is a torus, $T^{n}$, which we view as a quotient of $n$ dimensional real vector space, $\mathbb{R}^{n}$, by a lattice. In this example, if we endow $\mathbb{R}^{n}$ with a flat metric then $X=T^{n}$ has trivial holonomy group, since the Levi-Cevita connection is zero. Indeed, no matter which path we choose on $T^{n}$, the parallel transport of a 


\begin{tabular}{|c|ccccccc|}
\hline Manifold $X$ & $T^{n}$ & & $\mathrm{CY}_{3}$ & & $X_{G_{2}}$ & & $X_{\operatorname{Spin}(7)}$ \\
\hline \hline $\operatorname{dim}_{\mathbb{R}}(X)$ & $n$ & & 6 & & 7 & & 8 \\
\hline $\operatorname{Hol}(X)$ & $\mathbf{1}$ & $\subset$ & $S U(3)$ & $\subset$ & $G_{2}$ & $\subset$ & $\operatorname{Spin}(7)$ \\
\hline $\mathrm{SUSY}$ & 1 & $>$ & $1 / 4$ & $>$ & $1 / 8$ & $>$ & $1 / 16$ \\
\hline
\end{tabular}

Table 2: Relation between holonomy and supersymmetry for certain manifolds.

vector $\vec{v}$ along this path always brings it back to itself. Hence, this example is the most symmetric one, in the sense of the previous paragraph, $\operatorname{Hol}(X)=1$. Correspondingly, in $M$ theory, toroidal compactifications preserve all of the original supersymmetries.

Our next example is $\operatorname{Hol}(X)=S U(3)$ which corresponds to Calabi-Yau manifolds of complex dimension 3 (real dimension 6). These manifolds exhibit a number of remarkable properties, such as mirror symmetry, and are reasonably well studied both in the mathematical and in the physical literature. We just mention here that compactification on Calabi-Yau manifolds preserves $1 / 4$ of the original supersymmetry. In particular, compactification of heterotic string theory on $X=C Y_{3}$ yields an $\mathcal{N}=1$ effective field theory in $3+1$ dimensions.

The last two examples in Table 2 are $G_{2}$ and $\operatorname{Spin}(7)$ manifolds; that is, manifolds with holonomy group $G_{2}$ and $\operatorname{Spin}(7)$, respectively. They nicely fit into the general pattern, so that as we read Table 2 from left to right the holonomy increases, whereas the fraction of unbroken supersymmetry decreases. Specifically, compactification of $M$ theory on a manifold with $G_{2}$ holonomy leads to an $\mathcal{N}=1$ four-dimensional theory and is therefore of phenomenological interest. This is similar to the compactification of heterotic string theory on Calabi-Yau three-folds. Compactification on $\operatorname{Spin}(7)$ manifolds breaks supersymmetry even further.

Mathematically, the fact that all these manifolds preserve some supersymmetry is related to the existence of covariantly constant spinors:

$$
\nabla \xi=0
$$

In fact, with all bosonic fields apart from the metric set to zero, 2.10 is precisely the condition for unbroken supersymmetry in string or $M$ theory compactification. This condition on a spinor field automatically implies a holonomy reduction: since $\xi$ is invariant under parallel transport, $\operatorname{Hol}(g(X))$ must be such that the spinor representation contains the trivial representation. This is impossible if $\operatorname{Hol}(g(X))$ is $S O(n)$, since the spinor representation is irreducible. Therefore, $\operatorname{Hol}(g(X)) \subset S O(n)$. 
For example, if $\mathrm{Hol}(X)=G_{2}$ the covariantly constant spinor is the singlet in the decomposition of the spinor of $S O(7)$ into representations of $G_{2}$ :

$$
8 \rightarrow 7 \oplus \mathbf{1}
$$

Summarising, in Table 2 we listed some examples of special holonomies that will be discussed below. All of these manifolds preserve a certain fraction of supersymmetry, which depends on the holonomy group. Moreover, all of these manifolds are Ricci-flat,

$$
R_{i j}=0
$$

This useful property guarantees that all backgrounds of the form

$$
\mathbb{R}^{11-n} \times X
$$

automatically solve the eleven-dimensional Einstein equations with vanishing source terms for matter fields.

Of particular interest are $M$ theory compactifications on manifolds with exceptional holonomy,

$$
\begin{aligned}
& \text { M theory on } \quad \text { M theory on } \\
& G_{2} \text { manifold } \quad \operatorname{Spin}(7) \text { manifold } \\
& \Downarrow \quad \Downarrow \\
& \mathrm{D}=3+1 \text { QFT } \quad \mathrm{D}=2+1 \quad \mathrm{QFT}
\end{aligned}
$$

since they lead to effective theories with minimal supersymmetry in four and three dimensions, respectively. As mentioned in the introduction, in such theories one can find many interesting phenomena, e.g. confinement, dualities, rich phase structure, non-perturbative effects, etc. This rich structure makes minimal supersymmetry very attractive to study and, in particular, motivates the study of $M$ theory on manifolds with exceptional holonomy. In this context, the spectrum of elementary particles in the effective low-energy theory and their interactions are encoded in the geometry of the space $X$. Therefore, understanding the latter may help us to learn more about dynamics of minimally supersymmetric field theories, or even about $M$ theory itself!

\subsection{Invariant Forms and Minimal Submanifolds}

For a manifold $X$, we have introduced the notion of special holonomy and related it to the existence of covariantly constant spinors on $X, c f .(2.10)$. However, special holonomy manifolds can be also characerised by the existence of certain invariant forms and distinguished minimal submanifolds. 
Indeed, one can sandwich antisymmetric combinations of $\Gamma$-matrices with a covariantly constant spinor $\xi$ on $X$ to obtain antisymmetric tensor forms of various degree:

$$
\omega^{(p)}=\xi^{\dagger} \Gamma_{i_{1} \ldots i_{p}} \xi
$$

By construction, the $p$-form $\omega^{(p)}$ is covariantly constant and invariant under $\operatorname{Hol}(g(X))$. In order to find all possible invariant forms on a special holonomy manifold $X$, we need to decompose the space of differential forms on $X$ into irreducible representations of $\operatorname{Hol}(g(X))$ and identify all singlet components. Since the Laplacian of $g(X)$ preserves this decomposition the harmonic forms can also be decomposed this way. In a sense, for exceptional holonomy manifolds, the decomposition of cohomology groups into representations of $\mathrm{Hol}(g(X))$ is analogous to the Hodge decomposition in the realm of complex geometry.

For example, for a manifold with $G_{2}$ holonomy this decomposition is given by [17]:

$$
\begin{aligned}
& H^{0}(X, \mathbb{R})=\mathbb{R} \\
& H^{1}(X, \mathbb{R})=H_{\mathbf{7}}^{1}(X, \mathbb{R}) \\
& H^{2}(X, \mathbb{R})=H_{\mathbf{7}}^{2}(X, \mathbb{R}) \oplus H_{\mathbf{1 4}}^{2}(X, \mathbb{R}) \\
& H^{3}(X, \mathbb{R})=H_{\mathbf{1}}^{3}(X, \mathbb{R}) \oplus H_{\mathbf{7}}^{3}(X, \mathbb{R}) \oplus H_{\mathbf{2 7}}^{3}(X, \mathbb{R}) \\
& H^{4}(X, \mathbb{R})=H_{\mathbf{1}}^{4}(X, \mathbb{R}) \oplus H_{\mathbf{7}}^{4}(X, \mathbb{R}) \oplus H_{\mathbf{2 7}}^{4}(X, \mathbb{R}) \\
& H^{5}(X, \mathbb{R})=H_{\mathbf{7}}^{5}(X, \mathbb{R}) \oplus H_{\mathbf{1 4}}^{5}(X, \mathbb{R}) \\
& H^{6}(X, \mathbb{R})=H_{\mathbf{7}}^{6}(X, \mathbb{R}) \\
& H^{7}(X, \mathbb{R})=\mathbb{R}
\end{aligned}
$$

where $H_{\mathbf{n}}^{k}(X, \mathbb{R})$ is the subspace of $H^{k}(X, \mathbb{R})$ with elements in an $n$-dimensional irreducible representation of $G_{2}$. The fact that the metric on $X$ has irreducible $G_{2^{-}}$ holonomy implies global constraints on $X$ and this forces some of the above groups to vanish when $X$ is compact. For example a compact Ricci flat manifold with holonomy $S p(k), S U(k), G_{2}$ or $\operatorname{Spin}(7)$ has a finite fundamental group, $\pi_{1}(X)$. This implies that $H^{1}(X, \mathbb{R})=\mathbf{1}$, which in the $G_{2}$ case means

$$
H_{\mathbf{7}}^{k}(X, \mathbb{R})=0, \quad k=1, \ldots, 6
$$

Let us now return to the construction (2.12) of the invariant forms on $X$. From the above decomposition we see that on a $G_{2}$ manifold such forms can appear only in degree $p=3$ and $p=4$. They are called associative and coassociative forms, respectively. In fact, a coassociative 4-form is the Hodge dual of the associative 3-form. These forms, which we denote $\Phi$ and $* \Phi$, enjoy a number of remarkable properties. 
For example, the existence of $G_{2}$ holonomy metric on $X$ is equivalent to the closure and co-closure of the associative form ${ }^{1}$,

$$
\begin{aligned}
d \Phi & =0 \\
d * \Phi & =0 .
\end{aligned}
$$

This may look a little surprising, especially since the number of metric components on a 7-manifold is different from the number of components of a generic 3-form. However, given a $G_{2}$ holonomy metric,

$$
d s^{2}=\sum_{i=1}^{7} e^{i} \otimes e^{i},
$$

one can locally write the invariant 3 -form $\Phi$ in terms of the vielbein $e^{i}, c f .(2.6)$,

$$
\Phi=\frac{1}{3 !} \psi_{i j k} e^{i} \wedge e^{j} \wedge e^{k}
$$

where $\psi_{i j k}$ are the structure constants of the imaginary octonions (2.7).

It is, perhaps, less obvious that one can also locally reconstruct a $G_{2}$ metric from the assoiative 3 -form:

$$
\begin{aligned}
g_{i j} & =\operatorname{det}(B)^{-1 / 9} B_{i j} \\
B_{j k} & =-\frac{1}{144} \Phi_{j i_{1} i_{2}} \Phi_{k i_{3} i_{4}} \Phi_{i_{5} i_{6} i_{7}} \epsilon^{i_{1} \ldots i_{7}}
\end{aligned}
$$

This will be useful to us in the following sections.

Similarly, on a $\operatorname{Spin}(7)$ manifold $X$ we find only one invariant form (2.9) in degree $p=4$, called the Cayley form, $\Omega$. In this case, the decomposition of the cohomology groups of $X$ into $\operatorname{Spin}(7)$ representations is [17]:

$$
\begin{aligned}
& H^{0}(X, \mathbb{R})=\mathbb{R} \\
& H^{1}(X, \mathbb{R})=H_{\mathbf{8}}^{1}(X, \mathbb{R}) \\
& H^{2}(X, \mathbb{R})=H_{\mathbf{7}}^{2}(X, \mathbb{R}) \oplus H_{\mathbf{2 1}}^{2}(X, \mathbb{R}) \\
& H^{3}(X, \mathbb{R})=H_{\mathbf{8}}^{3}(X, \mathbb{R}) \oplus H_{\mathbf{4 8}}^{3}(X, \mathbb{R}) \\
& H^{4}(X, \mathbb{R})=H_{\mathbf{1}^{+}}^{4}(X, \mathbb{R}) \oplus H_{\mathbf{7}^{+}}^{4}(X, \mathbb{R}) \oplus H_{\mathbf{2 7}^{+}}^{4}(X, \mathbb{R}) \oplus H_{\mathbf{3 5}^{-}}^{4}(X, \mathbb{R}) \\
& H^{5}(X, \mathbb{R})=H_{\mathbf{8}}^{5}(X, \mathbb{R}) \oplus H_{\mathbf{4 8}}^{5}(X, \mathbb{R}) \\
& H^{6}(X, \mathbb{R})=H_{\mathbf{7}}^{6}(X, \mathbb{R}) \oplus H_{\mathbf{2 1}}^{6}(X, \mathbb{R}) \\
& H^{7}(X, \mathbb{R})=H_{\mathbf{8}}^{7}(X, \mathbb{R}) \\
& H^{8}(X, \mathbb{R})=\mathbb{R}
\end{aligned}
$$

\footnotetext{
${ }^{1}$ Another, equivalent condition is to say that the $G_{2}$-structure $(g, \Phi)$ is torsion-free: $\nabla \Phi=0$.
} 


\begin{tabular}{|c|c|c|c|c|}
\hline $\operatorname{Hol}(X)$ & cycle $S$ & $p=\operatorname{dim}(S)$ & Deformations & $\operatorname{dim}(\mathrm{Def})$ \\
\hline \hline$S U(3)$ & SLAG & 3 & unobstructed & $b_{1}(S)$ \\
\hline$G_{2}$ & associative & 3 & obstructed & - \\
\cline { 2 - 5 } & coassociative & 4 & unobstructed & $b_{2}^{+}(S)$ \\
\hline $\operatorname{Spin}(7)$ & Cayley & 4 & obstructed & - \\
\hline
\end{tabular}

Table 3: Deformations of calibrated submanifolds.

The additional label " \pm " denotes self-dual/anti-self-dual four-forms, respectively. The cohomology class of the 4 -form $\Omega$ generates $H_{1^{+}}^{4}(X, \mathbb{R})$,

$$
H_{1^{+}}^{4}(X, \mathbb{R})=\langle[\Omega]\rangle
$$

Again, on a compact manifold with exactly $\operatorname{Spin}(7)$-holonomy we have extra constraints,

$$
H_{\mathbf{8}}^{1}=H_{\mathbf{8}}^{3}=H_{\mathbf{8}}^{5}=H_{\mathbf{8}}^{7}=0, \quad H_{\mathbf{7}}^{2}=H_{\mathbf{7}}^{4}=H_{\mathbf{7}}^{6}=0
$$

Another remarkable propety of the invariant forms is that they represent the volume forms of minimal submanifolds in $X$. The forms with these properties are called calibrations, and the corresponding submanifolds are called calibrated submanifolds [18]. More precisely, we say that a closed $p$-form $\Psi$ is a calibration if it is less than or equal to the volume on each oriented $p$-dimensional submanifold $S \subset X$. Namely, combining the orientation of $S$ with the restriction of the Riemann metric on $X$ to the subspace $S$, we can define a natural volume form $\operatorname{vol}\left(T_{x} S\right)$ on the tangent space $T_{x} S$ for each point $x \in S$. Then, $\left.\Psi\right|_{T_{x} S}=\alpha \cdot \operatorname{vol}\left(T_{x} S\right)$ for some $\alpha \in \mathbb{R}$, and we write:

$$
\left.\Psi\right|_{T_{x} S} \leq \operatorname{vol}\left(T_{x} S\right)
$$

if $\alpha \leq 1$. If equality holds for all points $x \in S$, then $S$ is called a calibrated submanifold with respect to the calibration $\Psi$. According to this definition, the volume of a calibrated submanifold $S$ can be expressed in terms of $\Psi$ as:

$$
\operatorname{Vol}(S)=\left.\int_{x \in S} \Psi\right|_{T_{x} S}=\int_{S} \Psi
$$

Since $d \Psi=0$ the right-hand side depends only on the cohomology class, so:

$$
\operatorname{Vol}(S)=\int_{S} \Psi=\int_{S^{\prime}} \Psi=\left.\int_{x \in S^{\prime}} \Psi\right|_{T_{x} S^{\prime}} \leq \int_{x \in S^{\prime}} \operatorname{vol}\left(T_{x} S^{\prime}\right)=\operatorname{Vol}\left(S^{\prime}\right)
$$


for any other submanifold $S^{\prime}$ in the same homology class. Therefore, we see that a calibrated submanifold has minimal volume in its homology class. This important property of calibrated submanifolds allows us to identify them with supersymmetric cycles, where the bound in volume becomes equivalent to the BPS bound. In particular, branes in string theory and $M$ theory wrapped over calibrated submanifolds can give rise to BPS states in the effective theory.

A familiar example of a calibrated submanifold is a special Lagrangian (SLAG) cycle in a Calabi-Yau 3-fold $X$. By definition, it is a 3-dimensional submanifold in $X$ calibrated with respect to the real part, $\operatorname{Re}(\Omega)$, of the holomorphic 3 -form $\Omega$ (more generally, $\operatorname{Re}\left(e^{i \theta} \Omega\right)$, where $\theta$ is an arbitrary phase). Another class of calibrated submanifolds in Calabi-Yau spaces consists of holomorphic subvarities, such as holomorphic curves, surfaces, etc. Similarly, if $X$ is a $G_{2}$ holonomy manifold, there are associative 3-manifolds and coassociative 4-manifolds, which correspond, respectively, to the associative 3 -form $\Phi$ and to the coassociative 4 -form $* \Phi$. In a certain sense, the role of these two types of calibrated submanifolds is somewhat similar to the holomorphic and special Lagrangian submanifolds in a Calabi-Yau space. In the case of Spin(7) holonomy manifolds, there is only one kind of calibrated submanifolds - called Cayley 4-manifolds - which correspond to the Cayley 4-form (2.9).

Deformations of calibrated submanifolds have been studied by Mclean [19], and are briefly summarised in Table 3. In particular, deformations of special Lagrangian and coassociative submanifolds are unobstructed in the sense that the local moduli space $\operatorname{Def}(S)$ has no singularities. In both cases, the dimension of the moduli space is determined by the topology of the calibrated submanifold $S$, viz. by $b_{1}(S)$ when $S$ is special Lagrangian, and by $b_{2}^{+}(S)$ when $S$ is coassociative. These two types of calibrated submanifolds will play a special role in what follows.

\subsection{Why Exceptional Holonomy is Hard}

Once we have introduced manifolds with special holonomy, let us try to explain why, until recently, so little was known about the exceptional cases, $G_{2}$ and $\operatorname{Spin}(7)$. Indeed, on the physics side, these manifolds are very natural candidates for constructing minimally supersymmetric field theories from string/ $M$ theory compactifications. Therefore, one might expect exceptional holonomy manifolds to be at least as popular and attractive as, say, Calabi-Yau manifolds. However, there are several reasons why exceptional holonomy appeared to be a difficult subject; here we will stress two of them:

\section{- Existence}




\section{- Singularities}

Let us now explain each of these problems in turn. The first problem refers to the existence of an exceptional holonomy metric on a given manifold $X$. Namely, it would be useful to have a general theorem which, under some favorable conditions, would guarantee the existence of such a metric. Indeed, Berger's classification, described earlier in this section, only tells us which holonomy groups can occur, but says nothing about examples of such manifolds or conditions under which they exist. To illustrate this further, let us recall that when we deal with Calabi-Yau manifolds we use such a theorem all the time - it is a theorem due to Yau, proving a conjecture due to Calabi, which guarantees the existence of a Ricci-flat metric on a compact, complex, Kähler manifold $X$ with $c_{1}(X)=0$ [20]. Unfortunately, no analogue of this theorem is known in the case of $G_{2}$ and $\operatorname{Spin}(7)$ holonomy (the local existence of such manifolds was first established in 1985 by Bryant [21]). Therefore, until such a general theorem is found we are limited to a case-by-case analysis of the specific examples. We will return to this problem in the next section. We also note that, to date, not a single example of a Ricci flat metric of special holonomy is known explicitly for a compact, simply connected manifold!

The second reason is associated with the singularities of these manifolds. As will be explained in the sequel, interesting physics occurs at the singularities. Moreover, the most interesting physics is associated with the types of singularities of maximal codimension, which exploit the geometry of the special holonomy manifold to the fullest. Until recently, little was known about these types of degenerations of manifolds with $G_{2}$ and $\operatorname{Spin}(7)$ holonomy. Moreover, even for known examples of isolated singularities, the dynamics of $M$ theory in these backgrounds was unclear. Finally, it is important to stress that the mathematical understanding of exceptional holonomy manifolds would be incomplete without a proper understanding of singular limits. 


\section{Construction of Manifolds With Exceptional Holon- omy}

In this section we review various methods of constructing compact and non-compact manifolds with $G_{2}$ and $\operatorname{Spin}(7)$ holonomy. In the absence of general existence theorems, akin to Yau's theorem [20], these methods become especially valuable. It is hard to

give full justice to all the existing techniques in one section. So, we will try to explain only a few basic methods, focusing mainly on those which played an important role in recent developments in string theory. We also illustrate these general techniques with several concrete examples that will appear in the later sections.

\subsection{Compact Manifolds}

The first examples of compact manifolds with $G_{2}$ and $\operatorname{Spin}(7)$ holonomy were constructed by Joyce [17]. The basic idea is to start with toroidal orbifolds of the form

$$
T^{7} / \Gamma \quad \text { or } \quad T^{8} / \Gamma
$$

where $\Gamma$ is a finite group, e.g. a product of $\mathbb{Z}_{2}$ cyclic groups. Notice that $T^{7}$ and $T^{8}$ themselves can be regarded as special cases of $G_{2}$ and $\operatorname{Spin}(7)$ manifolds, respectively. This is because their trivial holonomy group is a subgroup of $G_{2}$ or $\operatorname{Spin}(7)$. In fact, they possess continuous families of $G_{2}$ and $\operatorname{Spin}(7)$ structures. Therefore, if $\Gamma$ preserves one of these structures the quotient space automatically will be a space with exceptional holonomy. However, since the holonomy of the torus is trivial, the holonomy of the quotient is inherited from $\Gamma$ and is thus a discrete subgroup of $G_{2}$ or $\operatorname{Spin}(7)$. Joyce's idea was to take $\Gamma$ to act with fixed points, so that $T^{n} / \Gamma$ is a singular space, and then to repair the singularities to give a smooth manifold with continuous holonomy $G_{2}$ or $\operatorname{Spin}(7)$.

Example [17]: Consider a torus $T^{7}$, parametrized by periodic variables $x_{i} \sim x_{i}+1$, $i=1, \ldots, 7$. As we pointed out, it admits many $G_{2}$ structures. Let us choose one of them:

$\Phi=e^{1} \wedge e^{2} \wedge e^{3}+e^{1} \wedge e^{4} \wedge e^{5}+e^{1} \wedge e^{6} \wedge e^{7}+e^{2} \wedge e^{4} \wedge e^{6}-e^{2} \wedge e^{5} \wedge e^{7}-e^{3} \wedge e^{4} \wedge e^{7}-e^{3} \wedge e^{5} \wedge e^{6}$

where $e^{j}=d x_{j}$. Furthermore, let us take

$$
\Gamma=\mathbb{Z}_{2} \times \mathbb{Z}_{2} \times \mathbb{Z}_{2}
$$




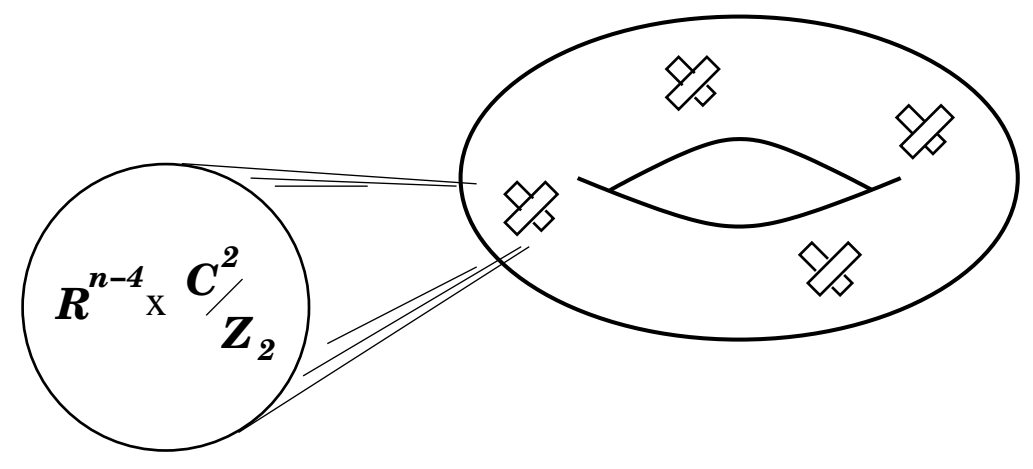

Figure 2: A cartoon representing Joyce orbifold $T^{n} / \Gamma$ with $\mathbb{C}^{2} / \mathbb{Z}_{2}$ orbifold points.

generated by three involutions

$$
\begin{aligned}
\alpha \quad: \quad\left(x_{1}, \ldots, x_{7}\right) & \mapsto\left(x_{1}, x_{2}, x_{3},-x_{4},-x_{5},-x_{6},-x_{7}\right) \\
\beta \quad: \quad\left(x_{1}, \ldots, x_{7}\right) & \mapsto\left(x_{1},-x_{2},-x_{3}, x_{4}, x_{5}, \frac{1}{2}-x_{6},-x_{7}\right) \\
\gamma \quad: \quad\left(x_{1}, \ldots, x_{7}\right) & \mapsto\left(-x_{1}, x_{2},-x_{3}, x_{4}, \frac{1}{2}-x_{5}, x_{6}, \frac{1}{2}-x_{7}\right)
\end{aligned}
$$

It is easy to check that these generators indeed satisfy $\alpha^{2}=\beta^{2}=\gamma^{2}=1$ and that the group $\Gamma=\langle\alpha, \beta, \gamma\rangle$ preserves the associative three-form $\Phi$ given above. It follows that the quotient space $X=T^{7} / \Gamma$ is a manifold with $G_{2}$ holonomy. More precisely, it is an orbifold since the group $\Gamma$ has fixed points of the form $T^{3} \times \mathbb{C}^{2} / \mathbb{Z}_{2}$. The existence of orbifold fixed points is a general feature of the Joyce construction.

In order to find a nice manifold $X$ with $G_{2}$ or $\operatorname{Spin}(7)$ holonomy one has to repair these singularities. In practice, this means removing the local neighbourhood of each singular point and replacing it with a smooth geometry, in a way which enhances the holonomy group from a discrete group to an exceptional holonomy group. This may be difficult (or even impossible) for generic orbifold singularities. However, if we have orbifold singularities that can also appear as degenerations of Calabi-Yau manifolds, then things simplify dramatically.

Suppose we have a $\mathbb{Z}_{2}$ orbifold, as in the previous example:

$$
\mathbb{R}^{n-4} \times \mathbb{C}^{2} / \mathbb{Z}_{2}
$$

where $\mathbb{Z}_{2}$ acts only on the $\mathbb{C}^{2}$ factor (by reflecting all the coordinates). This type of orbifold singularity can be obtained as a singular limit of the $A_{1} \mathrm{~A}$ (symptotically) $\mathrm{L}($ ocally) $\mathrm{E}($ uclidean) space, as we will see in detail in section 5:

$$
\mathbb{R}^{n-4} \times \mathrm{ALE}_{A_{1}} \quad \rightarrow \quad \mathbb{R}^{n-4} \times \mathbb{C}^{2} / \mathbb{Z}_{2}
$$


The ALE space has holonomy $S U(2)$, whereas its singular orbifold limit, being locally flat, has holonomy $\mathbb{Z}_{2} \subset S U(2)$. So we see that repairing the orbifold singularity enhances the holonomy from $\mathbb{Z}_{2}$ to $S U(2)$. An important point is that the ALE space is a non-compact Calabi-Yau 2-fold and we can use the tools of algebraic geometry to study its deformations. This is an important point; we used it implicitly to resolve the orbifold singularity. Moreover, Joyce proved that under certain conditions, resolving orbifold singularities in this way can be used to produce many manifolds of exceptional holonomy (3.21). Therefore, by the end of the day, when all singularities are removed, we can obtain a smooth, compact manifold $X$ with $G_{2}$ or $\operatorname{Spin}(7)$ holonomy.

Example: In the previous example, one finds a smooth manifold $X$ with $G_{2}$ holonomy and Betti numbers [17]:

$$
b^{2}(X)=12, \quad b^{3}(X)=43
$$

These come from the $\Gamma$-invariant forms on $T^{7}$ and also from the resolution of the fixed points on $T^{7} / \Gamma$. First, let us consider the invariant forms. It is easy to check that the there are no 1-forms and 2-forms on $T^{7}$ invariant under (3.22), and the only $\Gamma$-invariant 3 -forms are the ones that appear in the associative 3 -form $\Phi$,

$$
\Lambda_{\Gamma}^{3}\left(T^{7}\right)=\left\langle e^{123}, e^{145}, e^{167}, e^{246}, e^{257}, e^{347}, e^{356}\right\rangle
$$

where $e^{i j k}=e^{i} \wedge e^{j} \wedge e^{k}$. Therefore, we find $b_{\Gamma}^{1}\left(T^{7}\right)=b_{\Gamma}^{2}\left(T^{7}\right)=0$ and $b_{\Gamma}^{3}\left(T^{7}\right)=7$.

Now, let us consider the contribution of the fixed points to $b^{i}(X)$. We leave it to the reader to verify that the fixed point set of $\Gamma$ consists of 12 disjoint 3-tori, so that the singularity near each $T^{3}$ is of the orbifold type (3.23). Each singularity, modelled on $T^{3} \times \mathbb{C}^{2} / \mathbb{Z}_{2}$, can be resolved into a smooth space $T^{3} \times \mathrm{ALE}_{A_{1}}$. As will be explained in more detail in section 5 , via the resolution the second Betti number of the orbifold space $\mathbb{C}^{2} / \mathbb{Z}_{2}$ is increased by 1 . Therefore, by Kunneth formula, we find that $b^{4}\left(T^{3} \times \mathbb{C}^{2} / \mathbb{Z}_{2}\right)$ is increased by $1 \cdot b^{2}\left(T^{3}\right)=3$, while $b^{5}\left(T^{3} \times \mathbb{C}^{2} / \mathbb{Z}_{2}\right)$ jumps by $1 \cdot b^{3}\left(T^{3}\right)=1$. Using Poincaré duality and adding the contribution of all the fixed points and the $\Gamma$-invariant forms (3.25) together, we obtain the final resuls (3.24)

$$
\begin{aligned}
& b^{2}(X)=b_{\Gamma}^{2}\left(T^{7}\right)+12 \cdot 1=12 \\
& b^{3}(X)=b_{\Gamma}^{3}\left(T^{7}\right)+12 \cdot 3=43
\end{aligned}
$$

There are many other examples of the above construction, which are modelled not only on singularities of Calabi-Yau two-folds, but also on orbifold singularities of Calabi-Yau three-folds [17]. More examples can be found by replacing the tori in (3.21) 
by products of the $K 34$-manifold ${ }^{2}$ or Calabi-Yau three-folds with lower-dimensional tori. In such models, finite groups typically act as involutions on $K 3$ or Calabi-Yau manifolds, to produce fixed points of a familiar kind. Again, repairing the singularities using algebraic geometry techniques one can obtain compact, smooth manifolds with exceptional holonomy.

It may look a little disturbing that in Joyce's construction one always finds a compact manifold $X$ with exceptional holonomy near a singular (orbifold) limit. However, from the physics point of view, this is not a problem at all since interesting phenomena usually occur when $X$ develops a singularity. Indeed, as will be explained in more detail in section 4, compactification on a smooth manifold $X$ whose dimensions are very large (compared to the Planck scale) leads to a very simple effective field theory; it is abelian gauge theory with some number of scalar fields coupled to gravity. To find more interesting physics, such as non-abelian gauge symmetry or chiral matter, one needs singularities.

Moreover, there is a close relationship between various types of singularities and the effective physics they produce. A simple, but very important aspect of this relation is that a codimension $d$ singularity of $X$ can typically be associated with the physics of a $D \geq 11-d$ dimensional field theory. For example, there is no way one can obtain four-dimensional chiral matter or parity symmetry breaking in $D=3+1$ dimensions from a codimension four $\mathbb{C}^{2} / \mathbb{Z}_{2}$ singularity in $X$. As we will see, such singularities are associated with $D=7$ field theories.

Therefore, in order to reproduce properties specific to field theories in dimension four or three from compactification on $X$ one has to use the geometry of $X$ 'to the fullest' and consider singularities of maximal codimension. This motivates us to study isolated singular points in $G_{2}$ and $\operatorname{Spin}(7)$ manifolds.

Unfortunately, even though Joyce manifolds naturally admit orbifold singularities, none of them contains isolated $G_{2}$ or $\operatorname{Spin}(7)$ singularities close to the orbifold point in the space of metrics. Indeed, as we explained earlier, it is crucial that orbifold singularities are modelled on Calabi-Yau singularities, so that we can treat them using the familiar methods. Therefore, at best, such singularities can give us the same physics as one finds in the corresponding Calabi-Yau manifolds.

Apart from a large class of Joyce manifolds, very few explicit constructions of compact manifolds with exceptional holonomy are known. One nice approach was recently provided by A. Kovalev [22], where a smooth, compact 7-manifold $X$ with $G_{2}$ holonomy is obtained by gluing 'back-to-back' two asymptotically cylindrical Calabi-Yau

\footnotetext{
${ }^{2} K 3$ is the only compact 4-manifold admitting metrics with $S U(2)$ holonomy.
} 


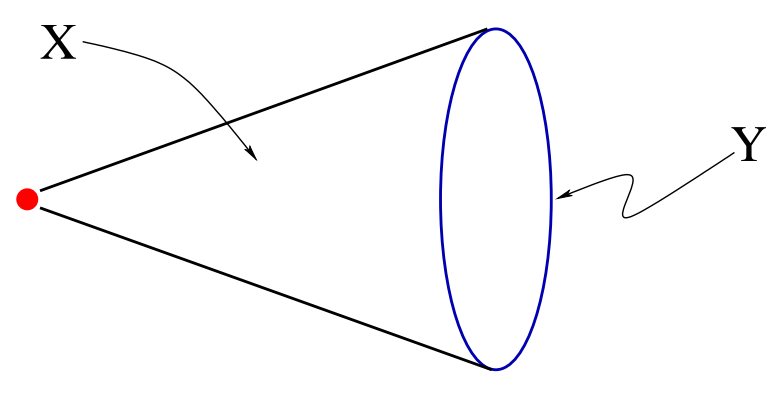

Figure 3: A cone over a compact space $Y$.

manifolds $W_{1}$ and $W_{2}$,

$$
X \cong\left(W_{1} \times S^{1}\right) \cup\left(W_{2} \times S^{1}\right)
$$

This construction is very elegant, but like Joyce's construction produces smooth $G_{2^{-}}$ manifolds. In particular, it would be interesting to study deformations of these spaces and to see if they can develop the kinds of isolated singularities of interest to physics. This leaves us with the following

Open Problem: Construct compact $G_{2}$ and $\operatorname{Spin}(7)$ manifolds with various types of isolated singularities

\section{$3.2 \quad$ Non-compact Manifolds}

As we will demonstrate in detail, interesting physics occurs at the singular points of the special holonomy manifold $X$. Depending on the singularity, one may find, for example, extra gauge symmetry or charged massless states localized at the singularity. Even though the physics depends strongly upon the details of the singularity itself, this physics typically depends only on properties of $X$ in the neighbourhood of the singularity. Therefore, in order to study the physics associated with a given singularity, one can imagine isolating the local neighbourhood of the singular point and studying it separately. In practice this means replacing the compact $X$ with a non-compact manifold with singularity and gives us the so-called 'local model' of the singular point. This procedure is somewhat analagous to considering one factor in the standard model gauge group, rather than studying the whole theory at once. In this sense, non-compact manifolds provide us with the basic building blocks for the low-energy energy physics that may appear in vacua constructed from compact manifolds.

Here we discuss a particular class of isolated singularities, namely conical singularies. They correspond to degenerations of the metric on the space $X$ of the form:

$$
d s^{2}(X)=d t^{2}+t^{2} d s^{2}(Y)
$$




\begin{tabular}{|c|c|c|}
\hline Holonomy & Topology of $X$ & Base $Y$ \\
\hline \hline \multirow{3}{*}{$G_{2}$} & $\mathbf{S}^{4} \times \mathbb{R}^{3}$ & $\mathbb{C P}^{3}$ \\
\cline { 2 - 3 } & $\mathbb{C P}^{2} \times \mathbb{R}^{3}$ & $S U(3) / U(1)^{2}$ \\
\cline { 2 - 3 } & $\mathbf{S}^{3} \times \mathbb{R}^{4}$ & $S U(2) \times S U(2)$ \\
\cline { 2 - 2 } & $T^{1,1} \times \mathbb{R}^{2}$ & \\
\hline \hline \multirow{3}{*}{$\operatorname{Spin}(7)$} & $\mathbf{S}^{4} \times \mathbb{R}^{4}$ & $S O(5) / S O(3)$ \\
\cline { 2 - 3 } & $\mathbb{C P}^{2} \times \mathbb{R}^{4}$ & $S U(3) / U(1)$ \\
\cline { 2 - 2 } & $\mathbf{S}^{5} \times \mathbb{R}^{3}$ & \\
\hline
\end{tabular}

Table 4: Asymptotically conical manifolds with $G_{2}$ and $\operatorname{Spin}(7)$ holonomy.

where a compact space $Y$ is the base of the cone; the dimension of $Y$ is one less than the dimension of $X$. $X$ has an isolated singular point at the tip of the cone $t=0$, except for the special case when $Y$ is a round sphere, $\mathbf{S}^{n-1}$, in which case (3.26) is just Euclidean space.

The conical singularities of the form (3.26) are among the simplest isolated singularities one could study, see Figure 3. In fact, the first examples of non-compact manifolds with $G_{2}$ and $\operatorname{Spin}(7)$ holonomy, obtained by Bryant and Salamon [23] and rederived by Gibbons, Page, and Pope [24], exhibit precisely this type of degeneration. Specifically, the complete metrics constructed in $[23,24]$ are smooth everywhere, and asymptotically look like (3.26), for various base manifolds $Y$. Therefore, they can be considered as smoothings of conical singularities. In Table 3 we list the currently known asymptotically conical (AC) complete metrics with $G_{2}$ and $\operatorname{Spin}(7)$ holonomy that were originally found in $[23,24]$ and more recently $[25,26]$.

The method of constructing $G_{2}$ and $\operatorname{Spin}(7)$ metrics originally used in [23, 24] was essentially based on the direct analysis of the conditions for special holonomy (2.10) or the Ricci-flatness equations,

$$
R_{i j}=0,
$$

for a particular metric ansatz. We will not go into details of this approach here since it relies on finding the right form of the ansatz and, therefore, is not practical for generalizations. Instead, following $[27,28]$, we will describe a very powerful approach, recently developed by Hitchin [29], which allows one to construct all the $G_{2}$ and $\operatorname{Spin}(7)$ manifolds listed in Table 3 (and many more !) in a systematic manner. Another advantage of this method is that it leads to first-order differential equations, which are 
much easier than the second-order Einstein equations (3.27).

Before we explain the basic idea of Hitchin's construction, notice that for all of the AC manifolds in Table 3 the base manifold $Y$ is a homogeneous quotient space

$$
Y=G / K
$$

where $G$ is some group and $K \subset G$ is a subgroup. Therefore, we can think of $X$ as being foliated by principal orbits $G / K$ over a positive real line, $\mathbb{R}_{+}$, as shown on Figure 4. A real variable $t \in \mathbb{R}_{+}$in this picture plays the role of the radial coordinate; the best way to see this is from the singular limit, in which the metric on $X$ becomes exactly conical, $c f$. eq. (3.26).

As we move along $\mathbb{R}_{+}$, the size and the shape of the principal orbit changes, but only in a way consistent with the symmetries of the coset space $G / K$. In particular, at some point the principal orbit $G / K$ may collapse into a degenerate orbit,

$$
B=G / H
$$

where symmetry requires

$$
G \supset H \supset K
$$

At this point (which we denote $t=t_{0}$ ) the "radial evolution" stops, resulting in a non-compact space $X$ with a topologically non-trivial cycle $B$, sometimes called a bolt. In other words, the space $X$ is contractible to a compact set $B$, and from the relation (3.30) we can easily deduce that the normal space of $B$ inside $X$ is itself a cone on $H / K$. Therefore, in general, the space $X$ obtained in this way is a singular space, with a conical singularity along the degenerate orbit $B=G / H$. However, if $H / K$ is a round sphere, then the space $X$ is smooth,

$$
H / K=\mathbf{S}^{k} \quad \Longrightarrow \quad X \text { smooth }
$$

This simply follows from the fact that the normal space of $B$ inside $X$ in such a case is non-singular, $\mathbb{R}^{k+1}(=$ a cone over $H / K)$. It is a good exercise to check that for all manifolds listed in Table 3, one indeed has $H / K=\mathbf{S}^{k}$, for some value of $k$. To show this, one should first write down the groups $G, H$, and $K$, and then find $H / K$.

The representation of a non-compact space $X$ in terms of principal orbits, which are homogeneous coset spaces is very useful. In fact, as we just explained, topology of $X$ simply follows from the group data (3.30). For example, if $H / K=\mathbf{S}^{k}$ so that $X$ is smooth, we have

$$
X \cong(G / H) \times \mathbb{R}^{k+1}
$$

However, this structure can be also used to find a $G$-nvariant metric on $X$. In order to do this, all we need to know are the groups $G$ and $K$. 


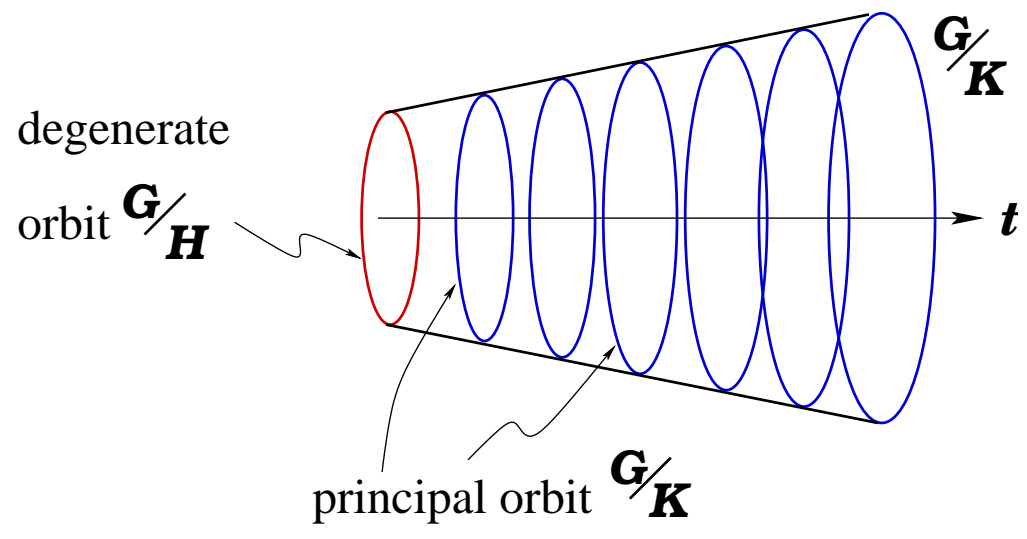

Figure 4: A non-compact space $X$ can be viewed as a foliation by principal orbits $Y=G / K$. The non-trivial cycle in $X$ correspond to the degenerate orbit $G / H$, where $G \supset H \supset K$.

First, let us sketch the basic idea of Hitchin's construction [29], and then explain the details in some specific examples. For more details and further applications we refer the reader to $[27,28]$. We start with a principal orbit $Y=G / K$ which can be, for instance, the base of the conical manifold that we want to construct. Let $\mathcal{P}$ be the space of (stable $\left.{ }^{3}\right) G$-invariant differential forms on $Y$. This space is finite dimensional and, moreover, it turns out that there exists a symplectic structure on $\mathcal{P}$. This important result allows us to think of the space $\mathcal{P}$ as the phase space of some dynamical system:

$$
\begin{gathered}
\mathcal{P}=\text { Phase Space } \\
\omega=\sum d x_{i} \wedge d p_{i}
\end{gathered}
$$

where we parametrized $\mathcal{P}$ by some coordinate variables $x_{i}$ and the conjugate momentum variables $p_{i}$.

Given a principal orbit $G / K$ and a space of $G$-invariant forms on it, there is a canonical construction of a Hamiltonian $H\left(x_{i}, p_{i}\right)$ for our dynamical system, such that the Hamiltonian flow equations are equivalent to the special holonomy condition [29]:

$$
\left\{\begin{aligned}
\frac{d x_{i}}{d t}=\frac{\partial H}{\partial p_{i}} \\
\frac{d p_{i}}{d t}=-\frac{\partial H}{\partial x_{i}}
\end{aligned} \Longleftrightarrow \begin{array}{cc}
\text { Special Holonomy Metric } \\
\text { on }\left(t_{1}, t_{2}\right) \times(G / K)
\end{array}\right.
$$

\footnotetext{
${ }^{3}$ Stable forms are defined as follows [30]. Let $X$ be a manifold of real dimension $n$, and $V=T X$. Then, the form $\rho \in \Lambda^{p} V^{*}$ is stable if it lies in an open orbit of the (natural) $G L(V)$ action on $\Lambda^{p} V^{*}$. In other words, this means that all forms in the neighborhood of $\rho$ are $G L(V)$-equivalent to $\rho$. This definition is useful because it allows one to define a volume. For example, a symplectic form $\omega$ is stable if and only if $\omega^{n / 2} \neq 0$.
} 
where the 'time' in the Hamiltonian system is identified with the radial variable $t$. Thus, solving the Hamiltonian flow equations from $t=t_{1}$ to $t=t_{2}$ with a particular boundary condition leads to the special holonomy metric on $\left(t_{1}, t_{2}\right) \times(G / K)$. Typically, one can extend the boundaries of the interval $\left(t_{1}, t_{2}\right)$ where the solution is defined to infinity on one side, and to a point $t=t_{0}$, where the principal orbit degenerates, on the other side. Then, this gives a complete metric with special holonomy on a non-compact manifold $X$ of the form (3.31). Let us now illustrate these general ideas in more detail in a concrete example.

Example: Let us take $G=S U(2)^{3}$ and $K=S U(2)$, the diagonal subgroup of $G$. We can form the following natural sequence of subgroups:

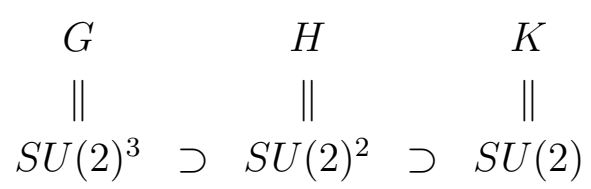

From the general formula (3.28) it follows that in this example we deal with a space $X$, whose principal orbits are

$$
Y=S U(2) \times S U(2) \cong \mathbf{S}^{3} \times \mathbf{S}^{3}
$$

Furthermore, $G / H \cong H / K \cong \mathbf{S}^{3}$ implies that $X$ is a smooth manifold with topology, cf. (3.31),

$$
X \cong \mathbf{S}^{3} \times \mathbb{R}^{4}
$$

In fact, $X$ is one of the asymptotically conical manifolds listed in Table 3.

In order to find a $G_{2}$ metric on this manifold, we need to construct the "phase space", $\mathcal{P}$, that is the space of $S U(2)^{3}$-invariant 3 -forms and 4-forms on $Y=G / K$ :

$$
\mathcal{P}=\Omega_{G}^{3}(G / K) \times \Omega_{G}^{4}(G / K)
$$

In this example, it turns out that each of the factors is one-dimensional, generated by a 3-form $\rho$ and by a 4 -form $\sigma$, respectively,

$$
\begin{gathered}
\rho=\sigma_{1} \sigma_{2} \sigma_{3}-\Sigma_{1} \Sigma_{2} \Sigma_{3}+x\left(d\left(\sigma_{1} \Sigma_{1}\right)+d\left(\sigma_{2} \Sigma_{2}\right)+d\left(\sigma_{3} \Sigma_{3}\right)\right), \\
\sigma=p^{2 / 5}\left(\sigma_{2} \Sigma_{2} \sigma_{3} \Sigma_{3}+\sigma_{3} \Sigma_{3} \sigma_{1} \Sigma_{1}+\sigma_{1} \Sigma_{1} \sigma_{2} \Sigma_{2}\right) .
\end{gathered}
$$

where we introduced two sets of left invariant 1-forms $\left(\sigma_{a}, \Sigma_{a}\right)$ on $Y$

$$
\begin{array}{lll}
\sigma_{1}=\cos \psi d \theta+\sin \psi \sin \theta d \phi, & \Sigma_{1}=\cos \tilde{\psi} d \tilde{\theta}+\sin \tilde{\psi} \sin \tilde{\theta} d \tilde{\phi} \\
\sigma_{2}=-\sin \psi d \theta+\cos \psi \sin \theta d \phi, & \Sigma_{2}=-\sin \tilde{\psi} d \tilde{\theta}+\cos \tilde{\psi} \sin \tilde{\theta} d \tilde{\phi} \\
\sigma_{3}=d \psi+\cos \theta d \phi, & \Sigma_{3}=d \tilde{\psi}+\cos \tilde{\theta} d \tilde{\phi}
\end{array}
$$


written explicitly in terms of the Euler angles $\psi, \tilde{\psi}, \theta, \tilde{\theta} \in[0, \pi]$ and $\phi, \tilde{\phi} \in[0,2 \pi]$. The invariant 1-forms $\sigma_{a}$ and $\Sigma_{a}$ satisfy the usual $S U(2)$ algebra

$$
d \sigma_{a}=-\frac{1}{2} \epsilon_{a b c} \sigma_{a} \wedge \sigma_{b}, \quad d \Sigma_{a}=-\frac{1}{2} \epsilon_{a b c} \Sigma_{a} \wedge \Sigma_{b}
$$

Therefore, we have only one "coordinate" $x$ and its conjugate "momentum" $p$, parametrizing the "phase space" $\mathcal{P}$ of our model. In order to see that there is a natural symplectic structure on $\mathcal{P}$, note that $x$ and $p$ multiply exact forms. For $x$ this obviously follows from (3.36) and for $p$ this can be easily checked using (3.37) and (3.39). This observation can be used to define a non-degenerate symplectic structure on $\mathcal{P}=\Omega_{\text {exact }}^{3}(Y) \times \Omega_{\text {exact }}^{4}(Y)$. Explicitly, it can be written as

$$
\omega\left(\left(\rho_{1}, \sigma_{1}\right),\left(\rho_{2}, \sigma_{2}\right)\right)=\left\langle\rho_{1}, \sigma_{2}\right\rangle-\left\langle\rho_{2}, \sigma_{1}\right\rangle,
$$

where, in general, for $\rho=d \beta \in \Omega_{\text {exact }}^{k}(Y)$ and $\sigma=d \gamma \in \Omega_{\text {exact }}^{n-k}(Y)$ one has a nondegenerate pairing

$$
\langle\rho, \sigma\rangle=\int_{Y} d \beta \wedge \gamma=(-1)^{k} \int_{Y} \beta \wedge d \gamma
$$

Once we have the phase space $\mathcal{P}$, it remains to write down the Hamiltonian flow equations (3.33). In Hitchin's construction, the Hamiltonian $H(x, p)$ is defined as an invariant functional on the space of differential forms, $\mathcal{P}$. Specifically, in the context of $G_{2}$ manifolds it is given by

$$
H=2 V(\sigma)-V(\rho)
$$

where $V(\rho)$ and $V(\sigma)$ are suitably defined volume functionals [29]. Even though the explicit form of the volume functionals $V(\sigma)$ and $V(\rho)$ is somewhat technical ${ }^{4}$, they can be systematically computed for any given choice of the forms $\sigma$ and $\rho$. Thus,

\footnotetext{
${ }^{4}$ The volume $V(\sigma)$ for a 4 -form $\sigma \in \Lambda^{4} T Y^{*} \cong \Lambda^{2} T Y \otimes \Lambda^{6} T Y^{*}$ is very easy to define. Indeed, we have $\sigma^{3} \in \Lambda^{6} T Y \otimes\left(\Lambda^{6} T Y^{*}\right)^{3} \cong\left(\Lambda^{6} T Y^{*}\right)^{2}$, and therefore we can take

$$
V(\sigma)=\int_{Y}\left|\sigma^{3}\right|^{\frac{1}{2}} .
$$

to be the volume for $\sigma$. In order to define the volume $V(\rho)$ for a 3-form $\rho \in \Lambda^{3} T Y^{*}$, one first defines a map $K_{\rho}: T Y \rightarrow T Y \otimes \Lambda^{6} T Y^{*}$, such that for a vector $v \in T Y$ it gives $K(v)=\imath(v) \rho \wedge \rho \in \Lambda^{5} T Y^{*} \cong$ $T Y \otimes \Lambda^{6} T Y^{*}$. Hence, one can define $\operatorname{tr}\left(K^{2}\right) \in\left(\Lambda^{6} T Y^{*}\right)^{2}$. Since stable forms with stabilizer $S L(3, \mathbb{C})$ are characterised by $\operatorname{tr}(K)^{2}<0$, following [30], we define

$$
V(\rho)=\int_{Y}\left|\sqrt{-t r K^{2}}\right|
$$


evaluating (3.41) for the $G$-invariant forms (3.36) and (3.37) we obtain the Hamiltonian flow equations:

$$
\left\{\begin{array}{l}
\dot{p}=x(x-1)^{2} \\
\dot{x}=p^{2}
\end{array}\right.
$$

These first-order equations can be easily solved, and the solution for $x(t)$ and $p(t)$ determines the evolution of the forms $\rho$ and $\sigma$, respectively. On the other hand, these forms define the associative three-form on the 7 -manifold $Y \times\left(t_{1}, t_{2}\right)$,

$$
\Phi=d t \wedge \omega+\rho,
$$

where $\omega$ is a 2-form on $Y$, such that $\sigma=\omega^{2} / 2$. For $x(t)$ and $p(t)$ satisfying the Hamiltonian flow equations the associative form $\Phi$ is automatically closed and coclosed, $c f$. (2.14). Therefore, as we explained in section 2 , it defines a $G_{2}$ holonomy metric. Specifically, one can use (2.17) to find the explicit form of the metric, which after a simple change of variables becomes the $G_{2}$ metric on the spin bundle over $\mathbf{S}^{3}$, originally found in $[23,24]$ :

$$
d s^{2}=\frac{d r^{2}}{1-r_{0}^{2} / r^{2}}+\frac{r^{2}}{12} \sum_{a=1}^{3}\left(\sigma_{a}-\Sigma_{a}\right)^{2}+\frac{r^{2}}{36}\left(1-\frac{r_{0}^{2}}{r^{2}}\right) \sum_{a=1}^{3}\left(\sigma_{a}+\Sigma_{a}\right)^{2}
$$

Here, $r>r_{0}$ and $r_{0}$ determines the size of the $\mathbf{S}^{3}$ generated by $\left(\sigma_{a}-\Sigma_{a}\right)$. It is easy to check that this $\mathbf{S}^{3}$ is an associative submanifold (with respect to the 3 -form (3.44)), and the total space is topologically $\mathbf{S}^{3} \times \mathbb{R}^{4}$.

The above example can be easily generalised in a number of directions. For example, if instead of (3.34) we take $G=S U(2)^{2}$ and $K$ to be its trivial subgroup, we end up with the same topology for $X$ and $Y$, but with a larger space of $G_{2}$ metrics on $X$. Indeed, for $G=S U(2)^{2}$ the space of $G$-invariant forms on $Y$ is much larger. Therefore, the corresponding dynamical system is more complicated and has a richer structure. Some specific solutions of this more general system have been recently constructed in $[31,32,33,34]$, but the complete solution is still not known.

There is a similar systematic method, also developed by Hitchin [29], of constructing complete non-compact manifolds with $\operatorname{Spin}(7)$ holonomy. Again, this method can be used to obtain the asymptotically conical metrics listed in Table 4, as well as other $\operatorname{Spin}(7)$ metrics recently found in $[35,36,25,37]$. Another approach to constructing special holonomy metrics is based on finding D6-brane solutions using gauged supergravity, following [38], see e.g. [39, 40, 41, 42]. Once can also use the technique of toric geometry to construct (singular) metrics on certain noncompact $G_{2}$ manifolds [43].

As a final remark we note, that this construction leads to non-compact exceptional holonomy manifolds with continuous isometries determined by the symmetry groups 
(3.30). In the effective low-energy field theory, these isometries play the role of the global symmetries. On the other hand, when such a manifold is realized as a part of a compact space $X$ with $G_{2}$ or $\operatorname{Spin}(7)$ holonomy, these isometries are broken by the global geometry of $X$. In particular, in either situation we can not use the geometric symmetries of $X$ to get non-abelian gauge fields in the effective field theory. 


\section{$4 M$ theory on Smooth Special Holonomy Mani- folds}

At low energies, $M$ theory is well approximated by eleven dimensional supergravity when spacetime is smooth and large compared to the eleven dimensional Planck length. So, when $X$ is smooth and large enough, we can obtain an effective low energy description by considering a Kaluza-Klein analysis of the fields on $X$. This analysis was first carried out in [44]. In this section, we review the Kaluza-Klein reduction of the eleven-dimensional supergravity on a manifolds with $G_{2}$ holonomy. The arguments for a reduction on a manifold with $\operatorname{Spin}(7)$ holonomy are very similar.

The supergravity theory has two bosonic fields, the metric $g$ and a 3-form field $C$ with field strength $G=d C$. The equation of motion for $C$ is $d * G=\frac{1}{2} G \wedge G$.

In compactification of eleven dimensional supergravity, massless scalars in four dimensions can originate from either the metric or the $C$-field. If $g(X)$ contains $k$ parameters i.e. there is a $k$-dimensional family of $G_{2}$-holonomy metrics on $X$, then there will be correspondingly $k$ massless scalars in four dimensions.

The scalars in four dimensions which originate from $C$ arise via the Kaluza-Klein ansatz,

$$
C=\sum \omega^{I}(x) \phi_{I}(y)+\ldots
$$

where $\omega^{I}$ form a basis for the harmonic 3-forms on $X$. These are zero modes of the Laplacian on $X$ (and are closed and co-closed). There are $b_{3}(X)$ linearly independent such forms. The dots refer to further terms in the Kaluza-Klein ansatz which will be prescribed later. The $\phi_{I}(y)$ are scalar fields in four dimensional Minkowski space with coordinates $y$. With this ansatz, these scalars are classically massless in four dimensions. To see this, note that,

$$
G=\sum \omega^{I} \wedge d \phi_{I}
$$

and $d * G$ is just

$$
d * G=\sum *_{7} \omega^{I} \wedge d *_{4} d \phi_{I}
$$

Since $G \wedge G$ vanishes to first order in the $C$-field modes, the $C$-field equation of motion actually aserts that the scalar fields $\phi_{I}$ are all massless in four dimensions. Thus, the $C$-field gives rise to $b_{3}(X)$ real massless scalars in four dimensions.

In fact it now follows from $\mathcal{N}=1$ supersymmetry in four dimensions that the KaluzaKlein analysis of $g$ will yield an additional $b_{3}(X)$ scalars in four dimensions. This is because the superpartners of $C$ should come from $g$ as these fields are superpartners in eleven dimensions. We should also add that (up to duality tranformations) all representations of the $\mathcal{N}=1$ supersymmetry algebra which contain one massless real 
scalar actually contain two scalars in total which combine into complex scalars. We will now describe how these scalars arise explicitly.

We began with a $G_{2}$-holonomy metric $g(X)$ on $X . g(X)$ obeys the vacuum Einstein equations,

$$
R_{i j}(g(X))=0
$$

To obtain the spectrum of modes originating from $g$ we look for fluctuations in $g(X)$ which also satisfy the vacuum Einstein equations. We take the fluctuations in $g(X)$ to depend on the four dimensional coordinates $y$ in Minkowski space. Writing the fluctuating metric as

$$
g_{i j}(x)+\delta g_{i j}(x, y)
$$

and expanding to first order in the fluctuation yields the Lichnerowicz equation

$$
\Delta_{L} \delta g_{i j} \equiv-\nabla_{M}^{2} \delta g_{i j}-2 R_{i m j n} \delta g^{m n}+2 R_{(i}^{k} \delta g_{j) k}=0
$$

Next we make a Kaluza-Klein ansatz for the fluctuations as

$$
\delta g_{i j}=h_{i j}(x) \rho(y)
$$

Note that the term $\nabla^{2}$ is the square of the full $d=11$ covariant derivative. If we separate this term into two

$$
\nabla_{M}^{2}=\nabla_{\mu}^{2}+\nabla_{i}^{2}
$$

we see that the fluctuations are scalar fields in four dimensions with squared masses given by the eigenvalues of the Lichnerowicz operator acting on the $h_{i j}$ :

$$
h_{i j} \nabla_{\mu}^{2} \rho(y)=-\left(\Delta_{L} h_{i j}\right) \rho(y)=-\lambda h_{i j} \rho(y)
$$

Thus, zero modes of the Lichnerowicz operator give rise to massless scalar fields in four dimensions. We will now show that we have precisely $b_{3}(X)$ such zero modes.

On a 7 -manifold of $S O(7)$ holonomy, the $h_{i j}$ — being symmetric 2-index tensors transform in the $\mathbf{2 7}$ dimensional representation. Under $G_{2}$ this representation remains irreducible. On the other hand, the 3 -forms on a $G_{2}$-manifold, which are usually in the 35 of $S O(7)$ decompose under $G_{2}$ as

$$
35 \longrightarrow 1+7+27
$$

Thus, the $h_{i j}$ can also be regarded as 3-forms on $X$. Explicitly,

$$
\Phi_{n[p q} h_{r]}^{n}=\omega_{p q r}
$$


The $\omega$ 's are 3 -forms in the same representation as $h_{i j}$ since $\Phi$ is in the trivial representation. The condition that $h$ is a zero mode of $\Delta_{L}$ is equivalent to $\omega$ being a zero mode of the Laplacian:

$$
\Delta_{L} h=0 \leftrightarrow \Delta \omega=0
$$

This shows that there are precisely $b_{3}(X)$ additional massless scalar fields coming from the fluctuations of the $G_{2}$-holonomy metric on $X$.

As we mentioned above, these scalars combine with the $\phi$ 's to give $b_{3}(X)$ massless complex scalars, $\Phi^{I}(y)$, which are the lowest components of massless chiral superfields in four dimensions. There is a very natural formula for the complex scalars $\Phi^{I}(y)$. Introduce a basis $\alpha_{I}$ for the third homology group of $X, H_{3}(X, \mathbf{R})$. This is a basis for the incontractible holes in $X$ of dimension three. We can choose the $\alpha_{I}$ so that

$$
\int_{\alpha_{I}} \omega^{J}=\delta_{I}^{J}
$$

Since the fluctuating $G_{2}$-structure is

$$
\Phi^{\prime}=\Phi+\delta \Phi=\Phi+\sum \rho^{I}(y) \omega^{I}(x)
$$

we learn that

$$
\Phi^{I}(y)=\int_{\alpha_{I}}\left(\Phi^{\prime}+i C\right)
$$

The fluctuations of the four dimensional Minkowski metric give us the usual fluctuations of four dimensional gravity, which due to supersymmetry implies that the four dimensional theory is locally supersymmetric.

In addition to the massless chiral multiplets, we also get massless vector multiplets. The bosonic component of such a mulitplet is a massless abelian gauge field which arises from the $C$-field through the Kaluza-Klein ansatz,

$$
C=\sum \beta^{\alpha}(x) \wedge A_{\alpha}(y)
$$

where the $\beta$ 's are a basis for the harmonic 2 -forms and the $A$ 's are one-forms in Minkowski space i.e. Abelian gauge fields. Again, the equations of motion for $C$ imply that the $A$ 's are massless in four dimensions. This gives $b_{2}(X)$ such gauge fields. As with the chiral multiplets above, the fermionic superpartners of the gauge fields arise from the gravitino field. Note that we could have also included an ansatz giving 2 -forms in four dimensions by summing over harmonic 1 -forms on $X$. However, since $b_{1}(X)=0$, this does not produce any new massless fields in four dimensions.

We are now in a position to summarise the basic effective theory for the massless fields. The low energy effective theory is an $\mathcal{N}=1$ supergravity theory coupled to 
$b_{2}(X)$ abelian vector multiplets and $b_{3}(X)$ massless, neutral chiral multiplets. This theory is relatively uninteresting physically. In particular, the gauge group is abelian and there are no light charged particles. We will thus have to work harder to obtain the basic requisites of the standard model — non-Abelian gauge fields and chiral fermions - from $G_{2}$-compactifications. The basic point of the following two sections is to emphasise that these features emerge naturally from singularities in $G_{2}$-manifolds.

Using similar arguments one can derive the effective low energy description of $M$ theory on a manifold $X$ with $\operatorname{Spin}(7)$ holonomy, in the regime where the size of $X$ is large (compared to the Planck scale). In this case, one also finds abelian $\mathcal{N}=1$ gauge theory in three dimensions with some number of (neutral) matter fields, coupled to supergravity. Specifically, from the Kaluza-Klein reduction one finds $b_{2}$ abelian vector fields, $\mathcal{A}^{i}$, which come from the modes of the three-form field $C$, and $\left(b_{3}+b_{4}^{-}+1\right)$ scalars, $\phi^{a}$. Some of these scalar fields, namely $b_{3}$ of them, come from the $C$-field, whereas the others correspond to deformations of the Spin(7) structure.

A novel feature of compactification on manifolds with $\operatorname{Spin}(7)$ holonomy is that, due to the membrane anomaly $[45,46,47]$, one often has to study compactifications with non-trivial background $G$-flux. Recall that the membrane path-integral is well-defined only if the $G$-field satisfies the shifted quantization condition [47]

$$
\left[\frac{G}{2 \pi}\right]-\frac{\lambda}{2} \in H^{4}(X ; \mathbb{Z})
$$

where $\lambda(X)=p_{1}(X) / 2 \in H^{4}(X ; \mathbb{Z})$ is an integral class for a spin manifold $X$. If $\lambda$ is even, one may consistently set $G=0$. However, if $\lambda$ is not divisible by two as an element of $H^{4}(X ; \mathbb{Z})$, one must turn on a half-integral $G$-flux in order to have a consistent vacuum.

The background $G$-flux generates Chern-Simons terms and a superpotential $\mathcal{W}(\phi)$ in the effective field theory $[48,49,36,50,51,52]$ :

$$
\begin{gathered}
k_{i j}=\frac{1}{2 \pi} \int_{X} \beta_{i} \wedge \beta_{j} \wedge G \\
\mathcal{W}=\frac{1}{2 \pi} \int_{X} \Omega \wedge G
\end{gathered}
$$

Taking these terms into account, we may write the complete supersymmetric action at a generic point in the moduli space of $X$ :

$$
\begin{aligned}
S_{3 d} & =\int d^{3} x\left[\sum_{k} \frac{1}{4 g_{k}^{2}}\left(\mathcal{F}_{\mu \nu}^{k} \mathcal{F}^{k \mu \nu}+\bar{\psi}^{k} i \Gamma \cdot D \psi^{k}\right)+\frac{1}{2} \sum_{a, b} g_{a b}\left(\partial_{\mu} \phi^{a} \partial^{\mu} \phi^{b}+\bar{\chi}^{a} i \Gamma \cdot D \chi^{b}\right)-\right. \\
& \left.-\frac{1}{2} \sum_{a, b} g^{a b}\left(\frac{\partial \mathcal{W}(\phi)}{\partial \phi^{a}} \frac{\partial \mathcal{W}(\phi)}{\partial \phi^{b}}-\frac{1}{2} \frac{\partial^{2} \mathcal{W}(\phi)}{\partial \phi^{a} \partial \phi^{b}} \bar{\chi}^{a} \chi^{b}\right)\right]-\sum_{i, j} \frac{i k_{i j}}{4 \pi} \int\left(\mathcal{A}^{i} \wedge d \mathcal{A}^{j}+\bar{\psi}^{i} \psi^{j}\right)
\end{aligned}
$$


Here, $\psi^{i}$ are the gaugino fields, $\chi^{a}$ represent the fermionic superpartners of the scalar fields $\phi^{a}, g^{i}$ are the gauge couplings, and $g^{a b}$ denotes the scalar field metric. In this Lagrangian we suppressed the terms corresponding to interactions with supergravity.

Before we conclude this section, let us remark that similar techniques can be applied to non-compact manifolds $X$ with $G_{2}$ or $\operatorname{Spin}(7)$ holonomy. In such cases, instead of the Betti numbers $b_{k}$ one should use the dimension of the space of $L^{2}$-normalisable $k$-forms on $X$. 


\section{$5 \quad M$ theory Dynamics on Singular Special Holon- omy Manifolds}

Since compactification on smooth manifolds does not produce interesting physics — in particular, does not lead to realistic quantum field theories - one has to study dynamics of string theory and $M$ theory on singular $G_{2}$ manifolds. This is a very interesting problem which can provide us with many insights about the infra-red behaviour of minimally supersymmetric gauge theories and even about $M$ theory itself. The new physics one might find at the singularities of $G_{2}$ and $\operatorname{Spin}(7)$ manifolds, could be,

- New light degrees of freedom

- Extra gauge symmetry

- Restoration of continuous/discrete symmetry

- Topology changing transitions

Before one talks about the physics associated with $G_{2}$ and $\operatorname{Spin}(7)$ singularities, it would be nice to have a classification of all such degenerations. Unfortunately, this problem is not completely solved even for Calabi-Yau manifolds (apart from complex dimension two ), and seems even less promising for real manifolds with exceptional holonomy. Therefore, one starts with some simple examples.

One simple kind of singularity — which we already encountered in section 3.1 in the Joyce construction of compact manifolds with exceptional holonomy — is an orbifold singularity ${ }^{5}$. Locally, an orbifold singularity can be represented as a quotient of $\mathbb{R}^{n}$ by some discrete group $\Gamma$,

$$
\mathbb{R}^{n} / \Gamma
$$

In perturbative string theory, the physics associated with such singularities can be systematically extracted from the orbifold conformal field theory [54]. (See [55, 5, $56,57,58,59,60]$ for previous work on conformal field theories associated with $G_{2}$ manifolds, and [55, 5, 61] for CFT's associated with Spin(7) manifolds.) Typically, one finds new massless degrees of freedom localized at the orbifold singularity and other phenomena listed above. However, the CFT technique is not applicable for studying $M$ theory on singular $G_{2}$ and $\operatorname{Spin}(7)$ manifolds. Moreover, as we explained earlier, many interesting phenomena occur at singularities which are not of the orbifold

\footnotetext{
${ }^{5}$ The classification of local $G_{2}$-orbifold singularities is reviewed in [53].
} 
type, and to study the physics of those we need some new methods. In the rest of this section, we describe two particularly useful methods of analyzing $M$ theory dynamics on singularities of special holonomy manifolds, which are based on the duality with the heterotic and type IIA string, respectively.

\subsection{Low Energy Dynamics via Duality with the Heterotic String}

We start with a duality between $M$ theory and the heterotic string theory, which, among other things, will help us to understand the origin of non-Abelian degrees of freedom arising from certain orbifold singularities. We have known for some time now that non-Abelian gauge groups emerge from $M$ theory when space has a so-called ADE-singularity. We learned this in the context of the duality between $M$ theory on $K 3$ and the heterotic string on a flat three torus, $T^{3}$ [66]. So, our strategy for obtaining non-Abelian gauge symmetry from $G_{2}$ or $\operatorname{Spin}(7)$ compactifications will be to embed ADE-singularities into special holonomy manifolds. After reviewing the basic

features of the duality between $M$ theory on $K 3$ and heterotic string theory on $T^{3}$, we describe ADE-singularities explicitly. We then develop a picture of a $G_{2}$-manifold near an embedded ADE-singularity. Based on this picture we analyse what kinds of four dimensional gauge theories these singularities give rise to. We then go on to describe local models for such singular $G_{2}$-manifolds as finite quotients of smooth ones.

\section{$M$ theory - Heterotic Duality in Seven Dimensions}

$M$ theory compactified on a $K 3$ manifold is strongly believed to be equivalent to the heterotic string theory compactified on a 3-torus $T^{3}$. As with $G_{2}$ compactification, both of these are compactifications to flat Minkowski space. Up to diffeomorphisms, $K 3$ is the only simply connected, compact 4-manifold admitting metrics of $S U(2)$-holonomy. $S U(2)$ is the analog in four dimensions of $G_{2}$ in seven dimensions. Interestingly enough in this case $K 3$ is the only simply connected example, whereas there are many $G_{2^{-}}$ manifolds.

There is a 58-dimensional moduli space of $S U(2)$-holonomy metrics on $K 3$. This space $\mathcal{M}(K 3)$ is locally a coset space:

$$
\mathcal{M}(K 3)=\mathbf{R}^{+} \times \frac{S O(3,19)}{S O(3) \times S O(19)}
$$

An $S U(2)$ holonomy metric admits two parallel spinors, which when tensored with the 8 constant spinors of 7 -dimensional Minkowski space give 16 global supercharges. 
This corresponds to minimal supersymmetry in seven dimensions (in the same way that $G_{2}$-holonomy corresponds to minimal supersymmetry in four dimensions). If we work at a smooth point in $\mathcal{M}$ we can use Kaluza-Klein analysis and we learn immediately that the effective $\mathrm{d}=7$ supergravity has 58 massless scalar fields which parametrise $\mathcal{M}$. These are the fluctuations of the metric on $K 3$. Additionally, since $H^{2}(K 3, \mathbf{R}) \cong \mathbf{R}^{\mathbf{2 2}}$ there are twenty-two linearly independent classes of harmonic 2-forms. These may be used a la equation (33) to give a $U(1)^{22}$ gauge group in seven dimensions. We now go on to describe how this spectrum is the same as that of the heterotic string theory on $T^{3}$, at generic points in $\mathcal{M}$.

The heterotic string in ten dimensions has a low energy description in terms of a supergravity theory whose massless bosonic fields are a metric, a 2 -form $B$, a dilaton $\phi$ and non-Abelian gauge fields of structure group $S O(32)$ or $E_{8} \times E_{8}$. There are sixteen global supersymetries. Compactification on a flat $T^{3}$ preserves all supersymmetries which are all products of constant spinors on both $T^{3}$ and Minkowski space. A flat metric on $T^{3}$ involves six parameters so the metric gives rise to six massless scalars. and since there are three independent harmonic two forms we obtain from $B$ three more. The condition for the gauge fields to be supersymmetric on $T^{3}$ is that their field strengths vanish: these are so called flat connections. They are parametrised by Wilson lines around the three independent circles in $T^{3}$. These are representations of the fundamental group of $T^{3}$ in the gauge group. Since the fundamental group has three commuting generators we are looking for commuting triples of elements in $G$.

Most of the flat connections actually arise from Wilson loops which are actually in the maximal torus of the gauge group, which in this case is $U(1)^{16}$. Clearly, this gives a 48 dimensional moduli space giving 58 scalars altogether. Narain showed by direct computation that this moduli space is actually also locally the same form as $\mathcal{M}$ [62].

From the point of view of the heterotic string on $T^{3}$, the effective gauge group in 7 dimensions (for generic metric and $B$-field) is the subgroup of $S O(32)$ or $E_{8} \times E_{8}$ which commutes with the flat connection on $T^{3}$. At generic points in the moduli space of flat connections, this gauge group will be $U(1)^{16}$. This is because the generic flat connection defines three generic elements in $U(1)^{16} \subset G$. We can think of these as diagonal 16 by 16 matrices with all elements on the diagonal non-zero. Clearly, only the diagonal elements of $G$ will commute with these. So, at a generic point in moduli space the gauge group is abelian.

Six more $U(1)$ gauge fields arise as follows from the metric and $B$-field. $T^{3}$ has three harmonic one forms, so Kaluza-Klein reduction of $B$ gives three gauge fields. Additionally, since $T^{3}$ has a $U(1)^{3}$ group of isometries, the metric gives three more. In fact, the local action for supergravity theories in seven dimensions are actually determined by the number of massless vectors. So, in summary, we have shown that 
at generic points in $\mathcal{M}$ the low energy supergravity theories arising from $M$ theory on $K 3$ or heterotic string on $T^{3}$ are the same.

At special points, some of the eigenvalues of the flat connections will vanish. At these points the unbroken gauge group can get enhanced to a non-Abelian group. This is none other than the Higgs mechanism: the Higgs fields are just the Wilson lines. Additionally, because seven dimensional gauge theories are infrared trivial (the gauge coupling has dimension a positive power of length), the low energy quantum theory actually has a non-Abelian gauge symmetry ${ }^{6}$

If $M$ theory on $K 3$ is actually equivalent to the heterotic string in seven dimensions, it too should therefore exihibit non-Abelian symmetry enhancement at special points in the moduli space. These points are precisely the points in moduli space where the $K 3$ develops orbifold singularities. We will not provide a detailed proof of this statement, but will instead look at the $K 3$ moduli space in a neighbourhood of this singularity, where all the interesting behaviour of the theory is occuring. So, the first question is what do these orbifold singularities look like?

\section{ADE-singularities}

An orbifold singularity in a Riemannian 4-manifold can locally be described as $\mathbb{R}^{4} / \mathbb{T}$,

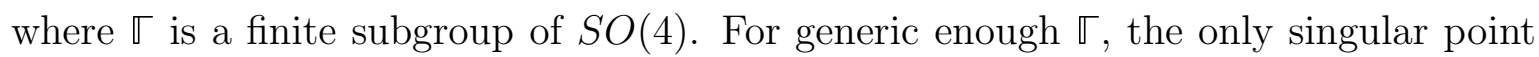
of this orbifold is the origin. These are the points in $\mathbb{R}^{\mathbb{4}}$ left invariant under $\mathbb{}$. A very crucial point is that on the heterotic side, supersymmetry is completely unbroken all over the moduli space, so our orbifold singularities in $K 3$ should also preserve supersymmetry. This means that $\mathbb{~ i s ~ a ~ f i n i t e ~ s u b g r o u p ~ o f ~} S U(2) \subset S O(4)$. This $S U(2)$ is the holonomy group of the global $K 3$ manifold. The particular $S U(2)$ can easily be identified as follows. Choose some set of complex coordinates so that $\mathbb{C}^{2} \equiv$ $\mathbb{R}^{4}$. Then, a point in $\mathbb{C}^{2}$ is labelled by a 2-component vector. The $S U(2)$ in question acts on this vector in the standard way:

$$
\left(\begin{array}{l}
u \\
v
\end{array}\right) \longrightarrow\left(\begin{array}{ll}
a & b \\
c & d
\end{array}\right)\left(\begin{array}{l}
u \\
v
\end{array}\right)
$$

The finite subgroups of $S U(2)$ have a classification which may be described in terms of the simply laced semi-simple Lie algebras: $A_{n}, D_{k}, E_{6}, E_{7}$ and $E_{8}$. There are two infinite series corresponding to $S U(n+1)=\mathrm{A}_{\mathrm{n}}$ and $S O(2 k)=\mathrm{D}_{\mathrm{k}}$ and three exceptional subgroups corresponding to the three exceptional Lie groups of $E$-type. The subgroups,

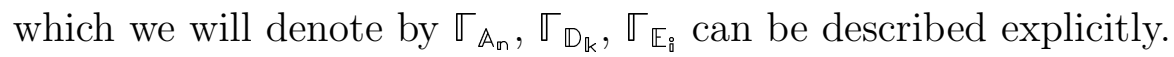

\footnotetext{
${ }^{6}$ Note that because of the dimensionful coupling constant, 7d Yang-Mills is ill defined in the UV. Here it is embedded into a consistent 11d theory which therefore provides a UV completion of this gauge theory. We will mainly be interested in low energy properties in this article.
} 
$\mathbb{T}_{\mathbb{A}_{m-1}}$ is isomorphic to $\mathbb{Z}_{m}$ - the cyclic group of order $n$ - and is generated by

$$
\left(\begin{array}{cc}
e^{\frac{2 \pi i}{n}} & 0 \\
0 & e^{\frac{-2 \pi i}{n}}
\end{array}\right)
$$

$\mathbb{}_{\mathbb{D}_{\mathbb{k}}}$ is isomorphic to $\mathbb{D}_{\mathbb{k}-2}$ - the binary dihedral group of order $4 k-8$ - and has two generators $\alpha$ and $\beta$ given by

$$
\alpha=\left(\begin{array}{cc}
e^{\frac{\pi i}{k-2}} & 0 \\
0 & e^{\frac{-\pi i}{k-2}}
\end{array}\right) \quad \beta=\left(\begin{array}{cc}
0 & i \\
i & 0
\end{array}\right)
$$

$\mathbb{T}_{\mathbb{E}_{\mathscr{6}}}$ is isomorphic to $\mathbb{T}$ - the binary tetrahedral group of order 24 - and has two generators given by

$$
\left(\begin{array}{cc}
e^{\frac{\pi i}{2}} & 0 \\
0 & e^{\frac{-\pi i}{2}}
\end{array}\right) \text { and } \frac{1}{\sqrt{2}}\left(\begin{array}{cc}
e^{\frac{2 \pi i 7}{8}} & e^{\frac{2 \pi i 7}{8}} \\
e^{\frac{2 \pi i 5}{8}} & e^{\frac{2 \pi i}{8}}
\end{array}\right)
$$

$\mathbb{T}_{\mathbb{E}_{7}}$ is isomorphic to $\mathbb{O}$ - the binary octohedral group of order 48 - and has three generators. Two of these are the generators of $\mathbb{T}$ and the third is

$$
\left(\begin{array}{cc}
e^{\frac{2 \pi i}{8}} & 0 \\
0 & e^{\frac{2 \pi i 7}{8}}
\end{array}\right)
$$

Finally, $\mathbb{\mathbb { E }}_{\mathbb{E}_{\mathbb{B}}}$ is isomorphic to $\mathbb{-}$ - the binary icosahedral group of order 120 - and has two generators given by

$$
-\left(\begin{array}{cc}
e^{\frac{2 \pi i 3}{5}} & 0 \\
0 & e^{\frac{2 \pi i 2}{5}}
\end{array}\right) \text { and } \frac{1}{e^{\frac{2 \pi i 2}{5}}-e^{\frac{2 \pi i 3}{5}}}\left(\begin{array}{cc}
e^{\frac{2 \pi i}{5}}+e^{\frac{-2 \pi i}{5}} & 1 \\
1 & -e^{\frac{2 \pi i}{5}}-e^{\frac{-2 \pi i}{5}}
\end{array}\right)
$$

Since all the physics of interest is happening near the orbifold singularities of $K 3$, we

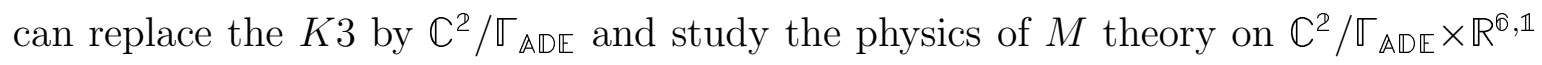
near its singular set which is just $\mathbf{0} \times \mathbb{R}^{6, \mathbb{1}}$. Since the $K 3$ went from smooth to singular as we varied its moduli we expect that the singular orbifolds $\mathbb{C}^{2} / \mathbb{\varpi}_{\mathbb{A D E}}$ are singular limits of non-compact smooth 4-manifolds $X^{A D E}$. Because of supersymmetry, these should have $S U(2)$-holonomy. This is indeed the case. The metrics of $S U(2)$-holonomy on the $X^{A D E}$ are known as ALE-spaces, since they asymptote to the locally Euclidean metric on $\mathbb{C}^{2} / \mathbb{\varpi}_{\mathbb{A D E}}$. Their existence was proven by Kronheimer [63] - who constructed a gauge theory whose Higgs branch is precisely the $X^{A D E}$ with its $S U(2)$-holonomy (or hyper-Kahler) metric. 
A physical description of this gauge theory arises in string theory. Consider Type IIA or IIB string theory on $\mathbb{C}^{2} / \mathbb{T}_{\mathbb{A D E}} \times \mathbb{R}^{5, \mathbb{1}}$. Take a flat $\mathrm{D} p$-brane (with $p \leq 5$ ) whose world-volume directions span $\mathbb{R}^{\mathbb{p}, \mathbb{1}} \subset \mathbb{R}^{5, \mathbb{1}}$ i.e. the D-brane is sitting at a point on the orbifold. Then the world-volume gauge theory, which was first derived in [64]., is given by the Kronheimer gauge theory. This theory has eight supersymmetries which implies that its Higgs branch is a hyper-Kahler manifold. For one D-brane this theory has a gauge group which is a product of unitary groups of ranks given by the Dynkin indices (or dual Kac labels) of the affine Dynkin diagram of the corresponding ADE-group. So, for the $A_{n}$-case the gauge group is $U(1)^{n+1}$. The matter content is also given by the affine Dynkin diagram - each link between a pair of nodes represents a hyper-multiplet transforming in the bi-fundamental representation of the two unitary groups. This is an example of a quiver gauge theory - a gauge theory determined by a quiver diagram.

We will make this explicit in the simplest case of $\mathbb{\square}_{\mathbb{A}_{\mathbb{1}}}{\mathbb{\mathbb { A } _ { \mathbb { A } }}}_{\mathbf{A}}$ is isomorphic to $\mathbb{Z}_{\mathbb{Z}}$ and is in fact the center of $S U(2)$. Its generator acts on $\mathbb{C}^{\mathbb{2}}$ as

$$
\left(\begin{array}{l}
u \\
v
\end{array}\right) \longrightarrow\left(\begin{array}{l}
-u \\
-v
\end{array}\right)
$$

In this case, the Kronheimer gauge theory has a gauge group which is $G=U(1)^{2}$ and has two fields $\Phi_{1}$ and $\Phi_{2}$ transforming as $(+,-)$ and $(-,+)$. These are hypermultiplets in the string theory realisation on a D-brane. Clearly, the diagonal $U(1)$ in $G$ acts trivially and so can be factored out to give a gauge group $G / U(1)=U(1)$ under which ( after rescaling the generator) $\Phi_{1}$ and $\Phi_{2}$ transform with charge +1 and -1 .

The hypermultiplets each contain two complex scalars $\left(a_{i}, b_{i}\right)$. The $a$ 's transform with charge +1 under $U(1)$, whilst the $b$ 's transform with charge -1 .

The potential energy of these scalar fields on the D-brane is

$$
V=|\vec{D}|^{2}=|\vec{\mu}|
$$

where the three $D$-fields $\vec{D}$ (which are also known as the hyper-Kahler moment maps $\vec{\mu}$ associated with $U(1)$ action on the $\mathbb{C}^{4}$ parameterised by the fields) are given by

$$
D_{1}=\left|a_{1}\right|^{2}+\left|b_{1}\right|^{2}-\left|a_{2}\right|^{2}-\left|b_{2}\right|^{2}
$$

and

$$
D_{2}+i D_{3}=a_{1} b_{1}-a_{2} b_{2}
$$

The space of zero energy minima of $V$ is the space of supersymmetric ground states $S$ of the theory on the brane up to gauge transformations:

$$
S=\{\vec{D}=0\} / U(1)
$$


In supersymmetric field theories, instead of solving these equations directly. it is equivalent to simply construct the space of gauge invariant holomorphic polynomials of the fields and impose only the holomorphic equation above (this is the $F$-term in the language of four dimensional supersymmetry). A solution to the $D_{1}$ equation is then guaranteed to exist because of invariance under the complexification of the gauge group.

In the case at hand the gauge invvariant polynomials are simply

$$
X=a_{1} b_{1} \quad Y=a_{2} b_{2} \quad Z=a_{1} b_{2} \quad W=a_{2} b_{1}
$$

These obviously parameterise $\mathbb{C}^{4}$ but are subject to the relation

$$
X Y=W Z
$$

However, the complex D-term equation asserts that

$$
X=Y
$$

hence

$$
X^{2}=W Z
$$

The space of solutions is precisely a copy of $\mathbb{C}^{2} / \mathbb{\square}_{\mathbb{A}_{\mathbb{1}}}$. To see this, we can parametrise $\mathbb{C}^{2} / \mathbb{T}_{\mathbb{A}_{\mathbb{1}}}$ algebraically in terms of the $\mathbb{}_{\mathbb{A}_{\mathbb{1}}}$ invariant coordinates on $\mathbb{C}^{2}$. These are $u^{2}, v^{2}$ and $u v$. If we denote these three coordinates as $w, z, x$, then obviously

$$
x^{2}=w z
$$

We prefer to re-write this equation by changing coordinates again. Defining $x=$ $u^{2}-v^{2}, y=i u^{2}+i v^{2}$ and $z=2 u v$ gives a map from $\mathbb{C}^{2} / \mathbb{\leftarrow}_{\mathbb{A}_{\mathbb{1}}}$ to $\mathbb{C}^{\mathfrak{B}}$. Clearly however,

$$
x^{2}+y^{2}+z^{2}=0
$$

which means that $\mathbb{C}^{2} / \mathbb{T}_{\mathbb{A}_{\mathbb{1}}}$ is the hypersurface in $\mathbb{C}^{\mathbb{3}}$ defined by this equation.

The orbifold can be deformed by adding a small constant to the right hand side,

$$
x^{2}+y^{2}+z^{2}=r^{2}
$$

If we take $x, y$ and $z$ to all be real and $r$ to be real then it is clear that the deformed 4 -manifold contains a 2-sphere of radius $r$. This 2 -sphere contracts to zero size as $r$ goes to zero. The total space of the deformed 4-manifold is in fact the co-tangent bundle of the 2 -sphere, $\mathbf{T}^{*} \mathbf{S}^{2}$. To see this write the real parts of the $x, y$ and $z$ as $x_{i}$ 
and their imaginary parts as $p_{i}$. Then, since $r$ is real, the $x_{i}$ are coordinates on the sphere which obey the relation

$$
\Sigma_{i} x_{i} p_{i}=0
$$

This means that the $p_{i}$ 's parametrise tangential directions. The radius $r$ sphere in the center is then the zero section of the tangent bundle. Since the manifold is actually complex it is natural to think of this as the co-tangent bundle of the Riemann sphere, $\mathbf{T}^{*} \mathbb{C} \mathbf{P}^{\mathbf{1}}$. In the context of Euclidean quantum gravity, Eguchi and Hanson constructed a metric of $S U(2)$-holonomy on this space, asymptotic to the locally flat metric on $\mathbb{C}^{2} / \mathbb{\varpi}_{\mathbb{A}_{\mathbb{1}}}$.

In the Kronheimer gauge theory on the D-brane, deforming the singularity corresponds to setting the D-terms or moment maps, not to zero but to constants. On the D-brane these cosntants represent the coupling of the background closed string fields to the brane. These fields parameterise precisely the metric moduli of the Eguchi-Hanson metric.

\section{$M$ theory Physics at The Singularity}

This metric, whose precise form we will not require actually has three parameters (since their are three $D$-terms in this case) which control the size and shape of the two-sphere which desingularises the orbifold. From a distance it looks as though there is an orbifold singularity, but as one looks more closely one sees that the singularity has been smoothened out by a two-sphere. The 2-sphere is dual to a compactly supported harmonic 2-form, $\alpha$. Thus, Kaluza-Klein reducing the $C$-field using $\alpha$ gives a $U(1)$ gauge field in seven dimensions. A vector multiplet in seven dimensions contains precisely one gauge field and three scalars and the latter are the parameters of the $\mathbf{S}^{\mathbf{2}}$. So, when $\mathbf{T}^{*} \mathbb{C} \mathbf{P}^{1}$ is smooth the massless spectrum is an abelian vector multiplet.

From the duality with the heterotic string we expect to see an enhancement in the gauge symmetry when we vary the scalars to zero i.e. when the sphere shrinks to zero size. In order for this to occur, $W^{ \pm}$-bosons must become massless at the singularity. These are electrically charged under the $U(1)$ gauge field which originated from $C$. From the eleven dimensional point of view the object which is charged under $C$ is the $M 2$-brane. The reason that this is natural is the equation of motion for $C$ is an eightform in eleven dimensions. A source for the $C$-field is thus generated by an 8-form closed current. In eleven dimensions such currents are naturally supported along three dimensional manifolds. These can be identified with $M 2$-brane world-volumes.

If the M2-brane wraps around the two-sphere, it appears as a particle from the seven dimensional point of view. This particle is electrically charged under the $U(1)$ and has a mass which is classically given by the volume of the sphere. Since, the $M 2$-brane has 
tension its dynamics will push it to wrap the smallest volume two-sphere in the space. This least mass configuration is in fact invariant under half of the supersymmetries 7 - a fact which means that it lives in a short representation of the supersymmetry algebra. This in turn means that its classical mass is in fact uncorrected quantum mechanically. The $M 2$-brane wrapped around this cycle with the opposing orientation has the opposing $U(1)$ charge to the previous one.

Thus, when the two-sphere shrinks to zero size we find two oppositely charged BPS multiplets become massless. These have precisely the right quantum numbers to enhance the gauge symmetry from $U(1)$ to $\mathrm{A}_{1}=S U(2)$. Super Yang-Mills theory in seven dimensions depends only on its gauge group, in the sense that its low energy lagrangian is uniquely determined by supersymmetry and the gauge group. In this case we are asserting that, in the absence of gravity, the low energy physics of $M$ theory on $\mathbb{C}^{\mathbb{2}} / \mathbb{\square}_{\mathbb{A}_{1}} \times \mathbb{R}^{\mathbb{6}, \mathbb{1}}$ is described by super Yang-Mills theory on $\mathbf{0} \times \mathbb{R}^{\mathbb{6}, \mathbb{1}}$ with gauge group $A_{1}$.

The obvious generalisation also applies: in the absence of gravity, the low energy

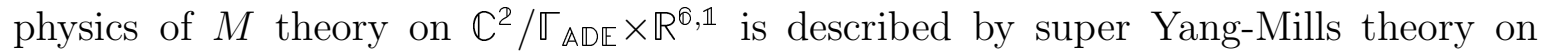
$\mathbf{0} \times \mathbb{R}^{6, \mathbb{1}}$ with $A D E$ gauge group. To see this, note that the smoothing out of the orbifold singularity in $\mathbb{C}^{\mathbb{P}} / \mathbb{T}_{\mathbb{A D E}}$ contains rank $(\mathrm{ADE})$ two-spheres which intersect according to the Cartan matrix of the ADE group. At smooth points in the moduli space the gauge group is thus $U(1)^{\text {rank(ADE) }}$. The corresponding wrapped membranes give rise to massive BPS multiplets with precisely the masses and quantum numbers required to enhance the gauge symmetry to the full ADE-group at the origin of the moduli space, where the orbifold singularity appears.

\section{ADE-singularities in $G_{2}$-manifolds.}

We have thus far restricted our attention to the ADE singularities in $K 3 \times \mathbb{R}^{6,1}$. However, the ADE singularity is a much more local concept. We can consider more complicated spacetimes $X^{10,1}$ with ADE singularities along more general seven-dimensional spacetimes, $Y^{6,1}$. Then, if $X$ has a modulus which allows us to scale up the volume of $Y$, the large volume limit is a semi-classical limit in which $X$ approaches the previous maximally symmetric situation discussed above. Thus, for large enough volumes we can assert that the description of the classical physics of $M$ theory near $Y$ is in terms of seven dimensional super Yang-Mills theory on $Y$ - again with gauge group determined by which ADE singularity lives along $Y$.

In the context of $G_{2}$-compactification on $X \times \mathbb{R}^{\mathbb{3}, \mathbb{1}}$, we want $Y$ to be of the form $W \times \mathbb{R}^{\S, \mathbb{1}}$, with $W$ the locus of ADE singularities inside $X$. Near $W \times \mathbb{R}^{\S, \mathbb{1}}, X \times \mathbb{R}^{\S, \mathbb{1}}$ looks like $\mathbb{C}^{\mathbb{2}} / \mathbb{\square}_{\mathbb{A D E}} \times W \times \mathbb{R}^{\mathfrak{X}, \mathbb{1}}$. In order to study the gauge theory dynamics without gravity,

\footnotetext{
${ }^{7}$ This is because the least volume two-sphere is an example of a calibrated or supersymmetric cycle.
} 
we can again focus on the physics near the singularity itself. So, we want to focus on seven-dimensional super Yang-Mills theory on $W \times \mathbb{R}^{\mathbb{B}, \mathbb{1}}$.

In flat space the super Yang-Mills theory has a global symmetry group which is $S O(3) \times S O(6,1)$. The second factor is the Lorentz group, the first is the R-symmetry. The theory has gauge fields transforming as $(\mathbf{1}, \mathbf{7})$, scalars in the $(\mathbf{3}, \mathbf{1})$ and fermions in the $(\mathbf{2}, \mathbf{8})$ of the universal cover. All fields transform in the adjoint representation of the gauge group. Moreover the sixteen supersymmetries also transform as $(\mathbf{2}, \mathbf{8})$.

On $W \times \mathbb{R}^{3,1}$ — with an arbitrary $W$ - the symmetry group gets broken to

$$
S O(3) \times S O(3)^{\prime} \times S O(3,1)
$$

Since $S O(3)^{\prime}$ is the structure group of the tangent bundle on $W$, covariance requires that the theory is coupled to a background $S O(3)^{\prime}$ gauge field - the spin connection on $W$. Similarly, though perhaps less intuitively, $S O(3)$ acts on the normal bundle to $W$ inside $X$, hence there is a background $S O(3)$ gauge field also.

The supersymmetries transform as $(\mathbf{2}, \mathbf{2}, \mathbf{2})+(\mathbf{2}, \mathbf{2}, \overline{\mathbf{2}})$. For large enough $W$ and at energy scales below the inverse size of $W$, we can describe the physics in terms of a four dimensional gauge theory. But this theory as we have described it is not supersymmetric as this requires that we have covariantly constant spinors on $W$. Because $W$ is curved, there are none. However, we actually want to consider the case in which $W$ is embedded inside a $G_{2}$-manifold $X$. In other words we require that our local model $\mathbb{C}^{2} / \mathbb{T}_{\mathrm{ADE}} \times W$ - admits a $G_{2}$-holonomy metric. When $W$ is curved this metric cannot be the product of the locally flat metric on $\mathbb{C}^{2} / \Gamma_{A D E}$ and a metric on $W$. Instead the metric is warped and is more like the metric on a fiber bundle in which the metric on $\mathbb{C}^{2}$ varies as we move around in $W$. Since the space has $G_{2}$-holonomy we should expect the four dimensional gauge theory to be supersymmetric. We will now demonstrate that this is indeed the case by examining the $G_{2}$-structure more closely. In order to do this however, we need to examine the $S U(2)$ structure on $\mathbb{C}^{2} / \llbracket$ as well.

4-dimensional spaces of $S U(2)$-holonomy are actually examples of hyperKahler manifolds. They admit three parallel 2 -forms $\omega_{i}$. These are analogous to the parallel forms on $G_{2}$-manifolds. These three forms transform locally under $S O(3)$ which locally rotates the complex structures. On $\mathbb{C}^{2}$ these forms can be given explicitly as

$$
\begin{aligned}
\omega_{1}+i \omega_{2} & =d u \wedge d v \\
\omega_{3} & =\frac{i}{2} d u \wedge d \bar{u}+d v \wedge d \bar{v}
\end{aligned}
$$

$\mathbb{\square}_{\mathbb{A D E}}$ is defined so that it preserves all three of these forms. The $S O(3)$ which rotates these three forms is identified with the $S O(3)$ factor in our seven dimensional gauge 
theory picture. This is because the moduli space of $S U(2)$-holonomy metrics is the moduli space of the gauge theory and this has an action of $S O(3)$.

In a locally flat frame we can write down a formula for the $G_{2^{-}}$structure on $\mathbb{C}^{2} / \mathbb{T}_{\mathbb{A D E}} \times W$,

$$
\Phi=\frac{1}{6} \omega_{i} \wedge e_{j} \delta^{i j}+e_{1} \wedge e_{2} \wedge e_{3}
$$

where $e_{i}$ are a flat frame on $W$. Note that this formula is manifestly invariant under the $S O(3)$ which rotates the $w_{i}$ provided that it also acts on the $e_{i}$ in the same way.

The key point is that when the $S O(3)$ of the gauge theory acts, in order for the $G_{2}$-structure to be well defined, the $e_{i}$ 's must transform in precisely the same way as the $\omega_{i}$. But $S O(3)^{\prime}$ acts on the $e_{i}$, because it is the structure group of the tangent bundle to $W$. Therefore, if $\mathbb{C}^{2} / \mathbb{\mathbb { A D E }}_{\mathrm{ADE}} \times W$, admits a $G_{2}$-holonomy metric, we must identify $S O(3)$ with $S O(3)^{\prime}$. In other words, the connection on the tangent bundle is identified with the connection on the normal $S O(3)^{\prime}$ bundle. This breaks the symmetries to the diagonal subgroup of the two $S O(3)$ 's and implies that the effective four dimensional field theory is classically supersymmetric. Identifying the two groups breaks the symmetry group down to $S O(3)^{\prime \prime} \times S O(3,1)$ under which the supercharges transform as $(\mathbf{1}, \mathbf{2})+(\mathbf{3}, \mathbf{2})+\mathbf{c c}$. We now have supersymmetries since the $(\mathbf{1}, \mathbf{2})$ and its conjugate can be taken to be constants on $W$.

An important point which we will not actually prove here, but will require in the sequel is that the locus of ADE-singularities - namely the copy of $W$ at the center of $\mathbb{C}^{2} / \mathbb{T}$ is actually a supersymmetric cycle i.e. calibrated by $\Phi$. This follows essentially from the fact that $\mathbb{A D D E}_{\mathbb{A}}$ fixes $W$ and therefore the $\Phi$ restricts to be the volume form on $W$. This is the condition for $W$ to be supersymmetric.

Supposing we could find a $G_{2}$-manifold of this type, what exactly is the four dimensional supersymmetric gauge theory it corresponds to? This we can answer also by Kaluza-Klein analysis [5, 6], since $W$ will be assumed to be smooth and 'large'. Under $S O(3)^{\prime \prime} \times S O(3,1)$, the seven dimensional gauge fields transform as $(\mathbf{3}, \mathbf{1})+(\mathbf{1}, \mathbf{4})$, the three scalars give $(\mathbf{3}, \mathbf{1})$ and the fermions give $(\mathbf{1}, \mathbf{2})+(\mathbf{3}, \mathbf{2})+\mathbf{c c}$. Thus the fields which are scalars under the four dimensional Lorentz group are two copies of the $\mathbf{3}$ of $S O(3)^{\prime \prime}$. These may be interpreted as two one forms on $W$. These will be massless if they are zero modes of the Laplacian on $W$ (wrt its induced metric from the $G_{2}$-manifold). There will be precisely $b_{1}(W)$ of these i.e. one for every harmonic one form. Their superpartners are clearly the $(\mathbf{3}, \mathbf{2})+\mathbf{c c}$ fermions, which will be massless by supersymmetry. This is precisely the field content of $b_{1}(W)$ chiral supermultiplets of the supersymmetry algebra in four dimensions.

The $(\mathbf{1}, \mathbf{4})$ field is massless if it is constant on $W$ and this gives one gauge field in four dimensions. The requisite superpartners are the remaining fermions which transform as $(\mathbf{1}, \mathbf{2})+\mathbf{c c}$. 
All of these fields transform in the adjoint representation of the seven dimensional gauge group. Thus the final answer for the massless fields is that they are described by $\mathcal{N}=1$ super Yang-Mills theory with $b_{1}(W)$ massless adjoint chiral supermultiplets. The case with pure "superglue" i.e. $b_{1}(W)=0$ is a particularly interesting gauge theory at the quantum level: in the infrared the theory is believed to confine colour, undergo chiral symmetry breaking and have a mass gap. We will actually exhibit some of these very interesting properties semi-classically in $M$ theory! Section 8 will be devoted to explaining this. But before we can do that we must first describe concrete examples of $G_{2}$-manifolds with the properties we desire.

One idea is to simply look for smooth $G_{2}$-manifolds which are topologically $\mathbb{C}^{2} \times W$ but admit an action of $S U(2)$ which leaves $W$ invariant but acts on $\mathbb{C}^{2}$ in the natural way. Then we simply pick a $\mathbb{I}_{\mathbb{A D E}} \subset S U(2)$ and form the quotient space $\mathbb{C}^{2} / \mathbb{\square}_{\mathbb{A D E}} \times W$.

Luckily, such non-compact $G_{2}$-manifolds were constructed some time ago in [23, 24], see section 3.2. Moreover, in these examples, $W=\mathbf{S}^{3}$, the simplest possible compact 3-manifold with $b_{1}(W)=0$.

\subsection{Low Energy Dynamics via IIA Duals}

Another useful method of analyzing $M$ theory on singular manifolds with special holonomy follows from the duality between type IIA string theory and $M$ theory compactified on a circle $[65,66]$ :

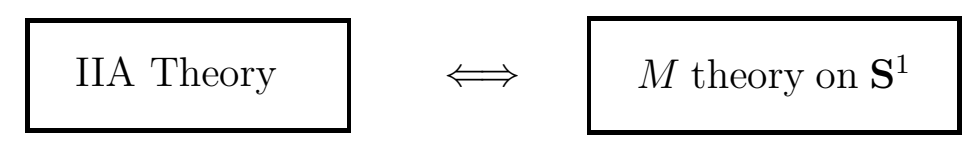

Among other things, this duality implies that any state in IIA theory can be identified with the corresponding state in $M$ theory. In this identification, some of the states acquire a geometric origin when lifted to eleven dimensions. In order to see this explicitly, let us write the eleven-dimensional metric in the form

$$
d s^{2}=e^{-\frac{2}{3} \phi} g_{i j} d x^{i} d x^{j}+e^{\frac{4}{3} \phi}\left(d x_{11}+A_{i} d x^{i}\right)^{2}
$$

Upon reduction to ten-dimensionsional space-time (locally parametrized by $x_{i}$ ), the field $\phi$ is identified with the dilaton, $A_{i}$ with Ramond-Ramond 1-form, and $g_{i j}$ with the ten-dimensional metric. Therefore, any IIA background that involves excitations of these fields uplifts to a purely geometric background in eleven dimensions. Moreover, from the explicit form of the metric (5.90) it follows that the eleven-dimensional geometry is a circle fibration over the ten-dimensional space-time, such that the topology 


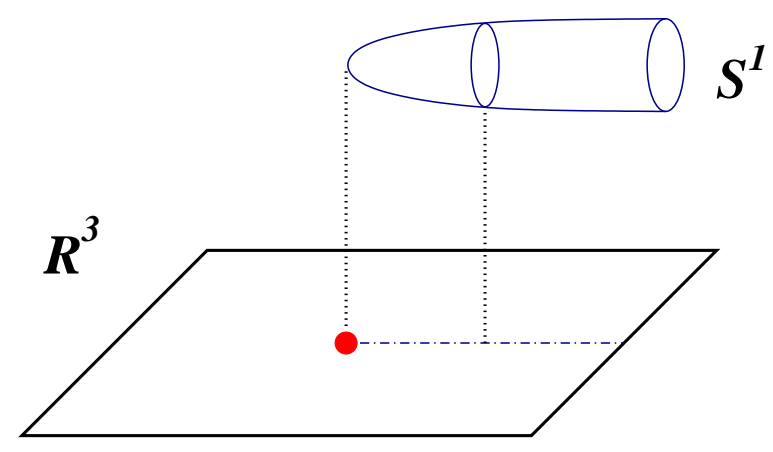

Figure 5: A cartoon representing Taub-NUT space as a circle fibration over a 3-plane.

of this fibration is determined by the Ramond-Ramond 1-form field. This important observation will play a central role in this section.

To be specific, let us consider a D6-brane in type IIA string theory. Since a D6brane is a source for the dilaton, for Ramond-Ramond 1-form, and for the metric, it is precisely the kind of state that uplifts to pure geometry. For example, if both D6-brane world-volume and the ambient space-time are flat, the dual background in $M$ theory is given by the Taub-NUT space:

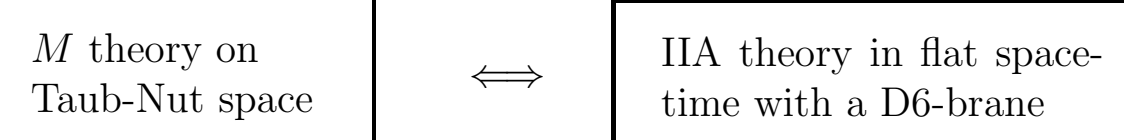

The Taub-NUT space is a non-compact four-manifold with $S U(2)$ holonomy. It can be viewed as a circle fibration over a 3-plane, see Figure 5 . The $\mathbf{S}^{1}$ fiber degenerates at a single point — the origin of the $\mathbb{R}^{3}$ — which is identified with the location of the D6-brane in type IIA string theory. On the other hand, at large distances the size of the ' $M$ theory circle' stabilizes at some constant value (related to the value of the string coupling constant in IIA theory). Explicitly, the metric on the Taub-NUT space is given by [67]:

$$
\begin{aligned}
d s_{T N}^{2} & =H d \vec{x}^{2}+H^{-1}\left(d x_{11}+A_{i} d x^{i}\right)^{2} \\
\vec{\nabla} \times \vec{A} & =-\vec{\nabla} H \\
H & =1+\frac{1}{2|\vec{x}|}
\end{aligned}
$$

This form of the metric makes especially clear the structure of the circle fibration. Indeed, if we fix a constant- $r$ sphere inside the $\mathbb{R}^{3}$, then it is easy to see that $\mathbf{S}^{1}$ fiber 
has 'winding number one' over this sphere. This indicates that there is a topological defect - namely, a D6-brane - located at $\vec{x}=0$, where $\mathbf{S}^{1}$ fiber degenerates.

The relation between D6-branes and geometry can be extended to more general manifolds that admit a smooth $U(1)$ action. Indeed, if $X$ is a space (not necessarily smooth and/or compact) with $U(1)$ isometry, such that $X / U(1)$ is smooth, then the fixed point set, $L$, of the $U(1)$ action must be of codimension 4 inside $X$ [68, 69]. This is just the right codimension to identify $L$ with the D6-brane locus ${ }^{8}$ in type IIA theory on $X / U(1)$ :

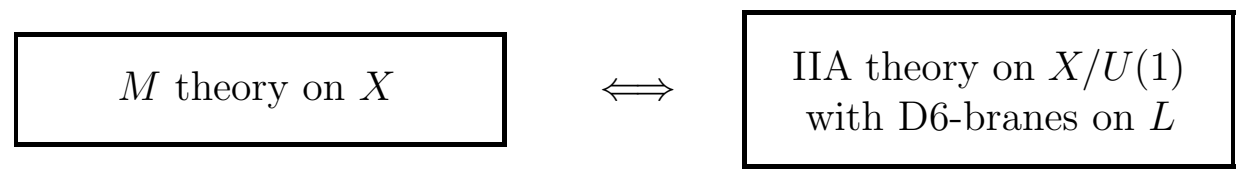

For example, if $X$ is the Taub-NUT space, then $U(1)$ action is generated by the shift of the periodic variable $x_{11}$ in (5.91). Dividing by this action one finds $X / U(1) \cong \mathbb{R}^{3}$ and that $L=\{\mathrm{pt}\} \in X$ indeed has codimension 4 .

It may happen that a space $X$ admits more than one $U(1)$ action. In that case, $M$ theory on $X$ will have several IIA duals, which may look very different but, of course, should exhibit the same physics. This idea was used by Atiyah, Maldacena, and Vafa to realise a geometric duality between certain IIA backgrounds as topology changing transitions in $M$ theory [7]. We will come back to this in section 7 .

Now let us describe a particularly useful version of the duality between $M$ theory on non-compact space $X$ and IIA theory with D6-branes on $X / U(1)$ which occurs when $X$ admits a $U(1)$ action, such that the quotient space is isomorphic to a flat Euclidean space. Suppose, for example, that $X$ is a non-compact $G_{2}$ manifold, such that

$$
X / U(1) \cong \mathbb{R}^{6}
$$

Then, the duality statement reads:
$M$ theory on non-compact
$G_{2}$ manifold $X$

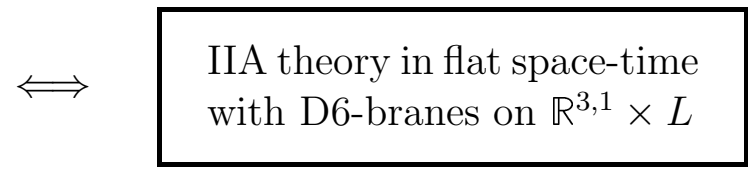

On the left-hand side of this equivalence the space-time in $M$ theory is $\mathbb{R}^{3,1} \times X$. On the other hand, the geometry of space-time in IIA theory is trivial (at least topologically) and all the interesting information about $X$ is mapped into the geometry of

\footnotetext{
${ }^{8}$ The part of the D-brane world-volume that is transverse to $X$ is flat and does not play an important rôle in our discussion here.
} 
the D6-brane locus $L$. For example, the Betti numbers of $L$ are determined by the corresponding Betti numbers of the space $X[36]$ :

$$
\begin{aligned}
& b_{k}(L)=b_{k+2}(X), \quad k>0 \\
& b_{0}(L)=b_{2}(X)+1
\end{aligned}
$$

Notice the shift in degree by 2, and also that the number of the D6-branes (= the number of connected components of $L$ ) is determined by the second Betti number of $X$. We will not present the derivation of this formula here. However, it is a useful exercise to check (e.g. using the Lefschetz fixed point theorem) that the Euler numbers of $X$ and $L$ must be equal, in agreement with (5.93).

The duality between $M$ theory on a non-compact manifold $X$ and a configuration of D6-branes in a (topologically) flat space can be used to study singular limits of $X$. Indeed, when $X$ develops a singularity, so does $L$. Moreover, $L$ must be a supersymmetric (special Lagrangian) submanifold in $\mathbb{R}^{6} \cong \mathbb{C}^{3}$ in order to preserve the same amount of supersymmetry ${ }^{9}$ as the $G_{2}$-holonomy space $X$. Therefore, the problem of studying the dynamics of $M$ theory on $G_{2}$ singularities can be recast as a problem of studying D6-brane configurations on singular special Lagrangian submanifolds in flat space [8].

Following Atiyah and Witten [8], let us see how this duality can help us to analyze one of the conical $G_{2}$ singularities listed in Table 4.

Example: Consider an asymptotically conical $G_{2}$ manifold $X$ with $S U(3) / U(1)^{2}$ principal orbits and topology

$$
X \cong \mathbb{C} \mathbf{P}^{2} \times \mathbb{R}^{3}
$$

Assuming that $M$ theory on this space $X$ admits a circle reduction to IIA theory with D6-branes in flat space, we can apply the general formula (5.93) to find the topology of the D6-brane locus L. For the manifold (5.94) we find the following non-zero Betti numbers:

\begin{tabular}{clll} 
Betti numbers of $X$ & & Betti numbers of $L$ \\
\hline$b_{4}=1$ & $\longrightarrow$ & $b_{2}=1$ \\
$b_{2}=1$ & $\longrightarrow$ & $b_{0}=2$
\end{tabular}

\footnotetext{
${ }^{9}$ In general, in a reduction from $M$ theory down to Type IIA one does not obtain the standard flat metric on $X / U(1) \cong \mathbb{R}^{6}$ due to non-constant dilaton and other fields in the background. However, one would expect that near the singularities of the D-brane locus $L$ these fields exhibit a regular behavior, and the metric on $X / U(1)$ is approximately flat, $c f$. [8]. In this case the condition for the Type IIA background to be supersymmetric can be expressed as a simple geometric criterion: it says that the D-brane locus $L$ should be a calibrated submanifold in $X / U(1)$.
} 


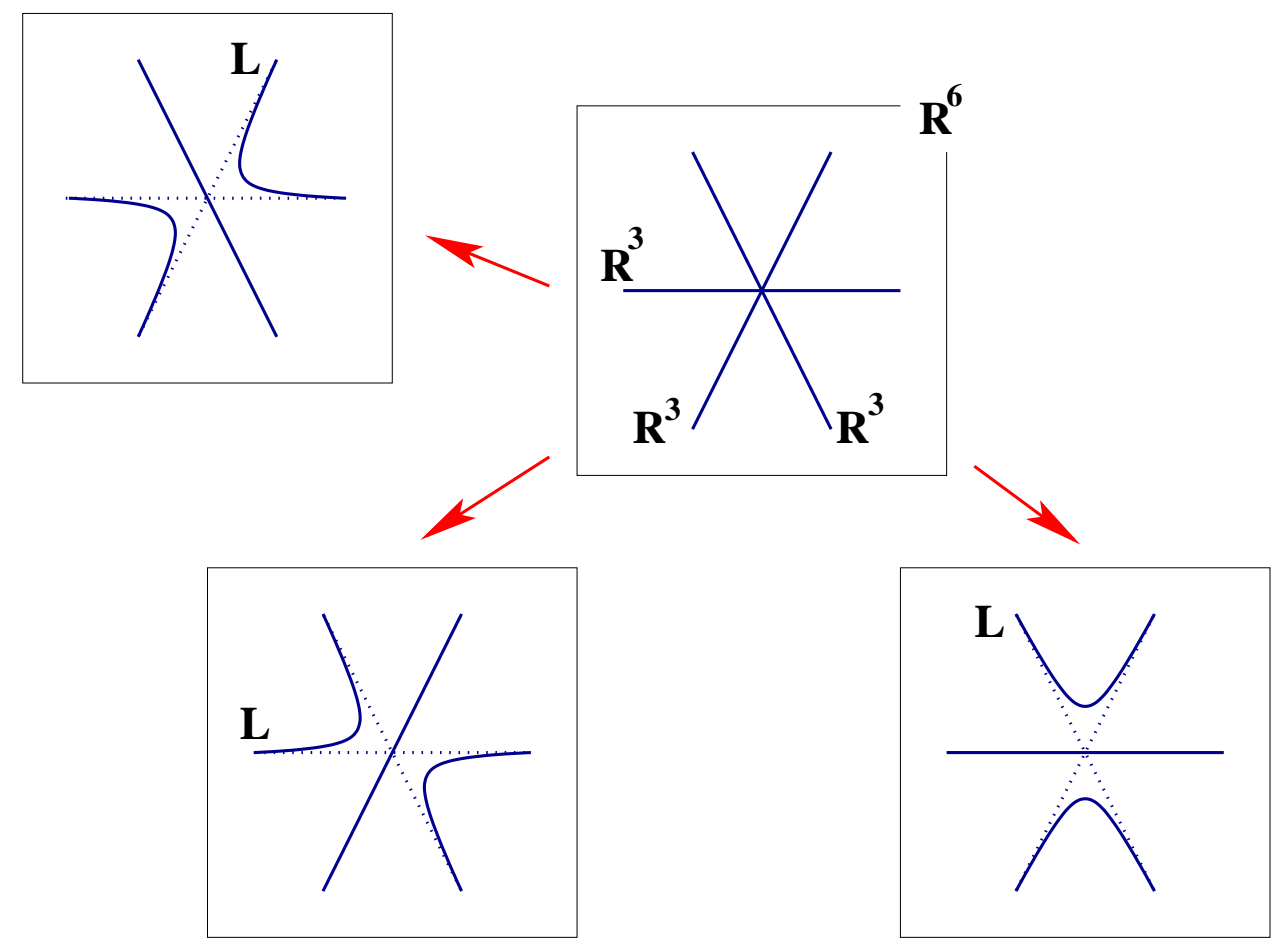

Figure 6: Intersection of special Lagrangian D6-branes dual to a $G_{2}$ cone over the flag manifold $S U(3) / U(1)^{2}$, and its three non-singular deformations. 
Therefore, we conclude that $L$ should be a non-compact 3-manifold with two connected components (since $b_{0}=2$ ) and with a single topologically non-trivial 2-cycle (since $b_{2}=1$ ). A simple guess for a manifold that has these properties is

$$
L \cong\left(\mathbf{S}^{2} \times \mathbb{R}\right) \cup \mathbb{R}^{3}
$$

It turns out that there indeed exists a special Lagrangian submanifold in $\mathbb{C}^{3}$ with the right symmetries and topology (5.95), see [8] for an explicit construction of the circle action on $X$ that has $L$ as a fixed point set. If we choose to parametrize $\operatorname{Re}\left(\mathbb{C}^{3}\right)=\mathbb{R}^{3}$ and $\operatorname{Im}\left(\mathbb{C}^{3}\right)=\mathbb{R}^{3}$ by 3 -vectors $\vec{\phi}_{1}$ and $\vec{\phi}_{2}$, respectively, then one can explicitly describe $L$ as the zero locus of the polynomial relations [71]:

$$
L=\left\{\vec{\phi}_{1} \cdot \vec{\phi}_{2}=-\left|\vec{\phi}_{1}\right|\left|\vec{\phi}_{2}\right| ; \quad\left|\vec{\phi}_{1}\right|\left(3\left|\vec{\phi}_{2}\right|^{2}-\left|\vec{\phi}_{1}\right|^{2}\right)=\rho\right\} \cup\left\{\left|\vec{\phi}_{1}-\sqrt{3} \vec{\phi}_{2}\right|=0\right\}
$$

It is easy to see that the first component of this manifold looks like a hyperboloid in $\mathbb{C}^{3}=\mathbb{R}^{3} \times \mathbb{R}^{3}$. It has a 'hole' in the middle, resulting in the $\mathbf{S}^{2} \times \mathbb{R}$ topology, required by (5.95). The second component in (5.96) is clearly a 3-plane, which goes through this hole, as shown in Figure 6.

When $X$ develops a conical singularity $L$ degenerates into a collection of three planes,

$$
L_{\text {sing }} \cong \mathbb{R}^{3} \cup \mathbb{R}^{3} \cup \mathbb{R}^{3}
$$

intersecting at a single point, see Figure 6 . This can be seen explicitly by taking $\rho \rightarrow 0$ limit in the geometry (5.96),

$$
\begin{array}{ll}
D 6_{1}: & \vec{\phi}_{1}=0 \\
D 6_{2}: & \frac{1}{2} \vec{\phi}_{1}+\frac{\sqrt{3}}{2} \vec{\phi}_{2}=0 \\
D 6_{3}: & -\frac{1}{2} \vec{\phi}_{1}+\frac{\sqrt{3}}{2} \vec{\phi}_{2}=0
\end{array}
$$

which corresponds to the limit of collapsing $\mathbf{S}^{2}$ cycle.

Therefore, in this example the physics of $M$ theory on conical $G_{2}$ singularity can be reduced to the physics of three intersecting D6-branes in type IIA string theory. In particular, since D6-branes appear symmetrically in this dual picture, one can conclude that there must be three ways of resolving this singularity, depending on which pair of D6-branes is deformed into a smooth special Lagrangian submanifold (5.96). This is precisely what Atiyah and Witten found in a more careful analysis [8]. We will come back to this example later, in section 7 . 
There is a similar duality that relates $M$ theory on $\operatorname{Spin}(7)$ manifolds to D6-brane configurations on coassociative cycles [70]. In particular, if $X$ is a non-compact $\operatorname{Spin}(7)$ manifold with a $U(1)$ action, such that

$$
X / U(1) \cong \mathbb{R}^{7}
$$

then one finds the following useful duality:

$$
\begin{aligned}
& M \text { theory on non-compact } \\
& \operatorname{Spin}(7) \text { manifold } X
\end{aligned}
$$$$
\Longleftrightarrow \quad \begin{aligned}
& \text { IIA theory in flat space-time } \\
& \text { with D6-branes on } \mathbb{R}^{3} \times L
\end{aligned}
$$

where $L$ is a coassociative submanifolds in $\mathbb{R}^{7}$. Again, on the left-hand side the geometry of space-time is $\mathbb{R}^{3} \times X$, whereas on the right-hand side space-time is (topologically) flat and all the interesting information is encoded in the geometry of the D6-brane locus $L$. Topology of the latter can be determined from the general formula (5.93). Therefore, as in the $G_{2}$ case, $M$ theory dynamics on singular $\operatorname{Spin}(7)$ manifolds can be obtained by investigating D6-brane configurations on singular coassociative submanifolds in flat space [36]. 


\section{Chiral Fermions from Conical Singularities in $G_{2^{-}}$ manifolds}

In the previous section we introduced various techniques for studying $M$ theory dynamics on singular manifolds, and discussed their application in a few simple examples. In particular, using duality with the heterotic string theory we explained the origin of the non-abelian gauge symmetries on ADE orbifold singularities, and using duality with the IIA string we derived the physics of $M$ theory at some simple conical singularities in $G_{2}$ and $\operatorname{Spin}(7)$ manifolds. Locally, a conical singularity can be described by the metric of the form (3.26), where $Y$ is the base of the cone ${ }^{10}$.

In this section, we continue the study of conical singularities using these methods. In particular, we will show that, for many different choices of $Y$, the dynamics of $M$ theory on a $G_{2}$ manifold $X$ with a conical singularity leads to chiral fermions, which are necessary for construction of realistic models of particle physics in four dimensions. We remind the reader that charged chiral fermions are important in nature since they are massless as long as the gauge symmetries they are charged under are unbroken. This enables us to understand, say, the lightness of the electron in terms of the Higgs vacuum expectation value and the Higgs-electron Yukawa coupling.

\subsection{Hints from Anomaly-Inflow.}

The basic strategy of this subsection will be to assume there is a $G_{2}$-manifold with a conical singularity of the above type and consider the variation of bulk terms in the $M$ theory effective action under various gauge symmetries. These will be shown to be nonzero if $Y$ obeys certain conditions. If the theory is to be consistent, these anomalous variations must be cancelled and this suggests the presence of chiral fermions in the spectrum. This is based upon [9] which also demonstrates that when $X$ is compact all these variations add up to zero!

The gauge symmetries we will consider are the ones we have focussed on in this paper: the $U(1)$ gauge symmetries from Kaluza-Klein reducing the $C$-field and the ADE symmetries from the ADE-singularities.

We begin with the former case. We take $M$ theory on $X \times \mathbb{R}^{\circledR, \mathbb{1}}$ with $X$ a cone on $Y$ so that $X=\mathbb{R} \times Y$. The Kaluza-Klein ansatz for $C$ which gives gauge fields in four dimensions is

$$
C=\sum \beta^{\alpha}(x) \wedge A_{\alpha}(y)
$$

\footnotetext{
${ }^{10}$ Known examples of $G_{2}$ and $\operatorname{Spin}(7)$ metrics that admit this kind of degeneration have been discussed in section 3.2 .
} 
where the $\beta$ 's are harmonic 2-forms on $X$. With this ansatz, consider the eleven dimensional Chern-Simons interaction

$$
S=\int_{X \times \mathbb{R}^{\mathfrak{s}, \mathbb{1}}} \frac{1}{6} C \wedge G \wedge G
$$

Under a gauge transformation of $C$ under which

$$
C \longrightarrow C+d \epsilon
$$

$S$ changes by something of the form ${ }^{11}$

$$
\delta S \sim \int_{X \times \mathbb{R}^{\mathbb{B}, \mathbb{1}}} d(\epsilon \wedge G \wedge G)
$$

We can regard $X$ as a manifold with boundary $\partial X=Y$ and hence

$$
\delta S \sim \int_{Y \times \mathbb{R}^{\mathcal{B}, \mathbb{1}}} \epsilon \wedge G \wedge G
$$

If we now make the Kaluza-Klein ansatz for the 2-form $\epsilon$

$$
\epsilon=\sum \epsilon^{\alpha} \beta^{\alpha}
$$

and use our ansatz for $C$, we find

$$
\delta S \sim \int_{Y} \beta^{\rho} \wedge \beta^{\sigma} \wedge \beta^{\delta} \int_{\mathbb{R}^{\beta, \mathbb{1}}} \epsilon^{\rho} d A^{\sigma} \wedge d A^{\delta}
$$

Thus if the integrals over $Y$ (which are topological) are non-zero we obtain a non-zero four dimensional interaction characteristic of an anomaly in an abelian gauge theory. Thus, if the theory is to be consistent, it is natural to expect a spectrum of chiral fermions at the conical singularity which exactly cancels $\delta S$.

We now turn to non-Abelian gauge anomalies. We have seen that ADE gauge symmetries in $M$ theory on a $G_{2}$-manifold $X$ are supported along a three-manifold $W$ in $X$. If additional conical singularities of $X$ are to support chiral fermions charges under the ADE-gauge group, then these singularities should surely also be points $P_{i}$ on $W$. So let us assume that near such a point, the metric on $X$ assumes the conical form. In four dimensional ADE gauge theories the triangle anomaly is only non-trivial for $A_{n}$-gauge groups. So, we restrict ourselves to this case. In this situation, there is a seven dimensional interaction of the form

$$
S=\int_{W \times \mathbb{R}^{3, \mathbb{1}}} K \wedge \Omega_{5}(A)
$$

\footnotetext{
${ }^{11}$ We will not be too careful about factors in this section.
} 
where $A$ is the $S U(n)$ gauge field and

$$
d \Omega_{5}(A)=\operatorname{tr} F \wedge F \wedge F
$$

$K$ is a two-form which is the field strength of a $U(1)$ gauge field which is part of the normal bundle to $W$. $K$ measures how the $\mathrm{A}_{\mathrm{n}}$-singularity twists around $W$. The $U(1)$ gauge group is the subgroup of $S U(2)$ which commutes with $\Gamma_{\mathrm{A}_{n}}$.

Under a gauge transformation,

$$
A \longrightarrow A+D_{A} \lambda
$$

and

$$
\delta S \sim \int_{W \times \mathbb{R}^{\beta, \mathbb{1}}}(K \wedge d \operatorname{tr} \lambda F \wedge F)
$$

so if $K$ is closed, $\delta S=0$. This will be the case if the $A_{n}$-singularity is no worse at the conical singularity $P$ than at any other point on $W$. If however, the $A_{n}$-singularity actually increases rank at $P$, then

$$
d K=2 \pi q \delta_{P}
$$

and we have locally a Dirac monopole of charge $q$ at $P$. The charge is an integer because of obvious quantisation conditions. In this situation we have that

$$
\delta S \sim \int_{W \times \mathbb{R}^{\boldsymbol{B}, \mathbb{1}}} d(K \wedge \operatorname{tr} \lambda F \wedge F)=-q \int_{\mathbb{R}^{\mathbb{3}, \mathbb{1}}} \operatorname{tr} \lambda F \wedge F
$$

which is precisely the triangle anomaly in an $S U(n)$ gauge theory. Thus, if we have this sort of situation in which the ADE-singularity along $W$ degenerates further at $P$ we also expect chiral fermions to be present.

We now go on to utilise the $M$ theory heterotic duality of section 5.1 to construct explicitly conically singular manifolds at which we know the existence of chiral fermions.

\subsection{Chiral Fermions via Duality With The Heterotic String}

In section 5.1 we utilised duality with the heterotic string on $T^{3}$ to learn about enhanced gauge symmetry in $M$ theory. We applied this to $G_{2}$-manifolds quite successfully. In this section we will take a similar approach. The following is based upon [10], see also $[72,73]$.

We start by considering duality with the heterotic string. The heterotic string compactified on a Calabi-Yau three-fold $Z$ can readily give chiral fermions. On the other hand, most Calabi-Yau manifolds participate in mirror symmetry. For $Z$ to participate in mirror symmetry means, according to Strominger, Yau and Zaslow [76] that, 
in a suitable limit of its moduli space, it is a $T^{3}$ fibration (with singularities and monodromies) over a base $W$. Then, taking the $T^{3}$ 's to be small and using on each fiber the equivalence of the heterotic string on $T^{3}$ with $M$ theory on $K 3$, it follows that the heterotic string on $Z$ is dual to $M$ theory on a seven-manifold $X$ that is $K 3$-fibered over $W$ (again with singularities and monodromies). $X$ depends on the gauge bundle on $Z$. Since the heterotic string on $Z$ is supersymmetric, $M$ theory on $X$ is likewise supersymmetric, and hence $X$ is a manifold of $G_{2}$ holonomy.

The heterotic string on $Z$ will typically have a four dimensional spectrum of chiral fermions. Since there are many $Z$ 's that could be used in this construction (with many possible classes of gauge bundles) it follows that there are many manifolds of $G_{2}$ holonomy with suitable singularities to give nonabelian gauge symmetry with chiral fermions. The same conclusion can be reached using duality with Type IIA, as many six-dimensional Type IIA orientifolds that give chiral fermions are dual to $M$ theory on a $G_{2}$ manifold [74].

Let us try to use this construction to determine what kind of singularity $X$ must have. (The reasoning and the result are very similar to that given in [75] for engineering matter from Type II singularities. In [75] the Dirac equation is derived directly rather than being motivated - as we will - by using duality with the heterotic string.) Suppose that the heterotic string on $Z$ has an unbroken gauge symmetry $G$, which we will suppose to be simply-laced (in other words, an A, D, or E group) and at level one. This means that each $K 3$ fiber of $X$ will have a singularity of type $G$. As one moves around in $X$ one will get a family of $G$-singularities parameterized by $W$. If $W$ is smooth and the normal space to $W$ is a smoothly varying family of $G$-singularities, the low energy theory will be $G$ gauge theory on $W \times \mathbb{R}^{\circledR, \mathbb{1}}$ without chiral multiplets. So chiral fermions will have to come from singularities of $W$ or points where $W$ passes through a worse-than-orbifold singularity of $X$.

We can use the duality with the heterotic string to determine what kind of singularities are required. The argument will probably be easier to follow if we begin with a specific example, so we will consider the case of the $\mathrm{E}_{8} \times \mathrm{E}_{8}$ heterotic string with $G=S U(5)$ a subgroup of one of the $E_{8}$ 's. Such a model can very easily get chiral 5's and 10's of $S U(5)$; we want to see how this comes about, in the region of moduli space in which $Z$ is $T^{3}$-fibered over $W$ with small fibers, and then we will translate this description to $M$ theory on $X$.

Let us consider, for example, the 5's. The commutant of $S U(5)$ in $\mathrm{E}_{8}$ is a second copy of $S U(5)$, which we will denote as $S U(5)^{\prime}$. Since $S U(5)$ is unbroken, the structure group of the gauge bundle $E$ on $Z$ reduces from $\mathrm{E}_{8}$ to $S U(5)^{\prime}$. Massless fermions in the heterotic string transform in the adjoint representation of $E_{8}$. The part of the adjoint representation of $\mathrm{E}_{8}$ that transforms as $\mathbf{5}$ under $S U(5)$ transforms as $\mathbf{1 0}$ under $S U(5)^{\prime}$. 
So to get massless chiral 5's of $S U(5)$, we must look at the Dirac equation $\mathcal{D}$ on $Z$ with values in the $\mathbf{1 0}$ of $S U(5)^{\prime}$; the zero modes of that Dirac equation will give us the massless 5's of the unbroken $S U(5)$.

We denote the generic radius of the $T^{3}$ fibers as $\alpha$, and we suppose that $\alpha$ is much less than the characteristic radius of $W$. This is the regime of validity of the argument for duality with $M$ theory on $X$ (and the analysis of mirror symmetry [76]). For small $\alpha$, we can solve the Dirac equation on $Z$ by first solving it along the fiber, and then along the base. In other words, we write $\mathcal{D}=\mathcal{D}_{T}+\mathcal{D}_{W}$, where $\mathcal{D}_{T}$ is the Dirac operator along the fiber and $\mathcal{D}_{W}$ is the Dirac operator along the base. The eigenvalue of $\mathcal{D}_{T}$ gives an effective "mass" term in the Dirac equation on $W$. For generic fibers of $Z \rightarrow W$, as we explain momentarily, the eigenvalues of $\mathcal{D}_{T}$ are all nonzero and of order $1 / \alpha$. This is much too large to be canceled by the behavior of $\mathcal{D}_{W}$. So zero modes of $\mathcal{D}$ are localized near points in $W$ above which $\mathcal{D}_{T}$ has a zero mode.

When restricted to a $T^{3}$ fiber, the $S U(5)^{\prime}$ bundle $E$ can be described as a flat bundle with monodromies around the three directions in $T^{3}$. In other words, as in section 5.1, we have three Wilson lines on each fiber. For generic Wilson lines, every vector in the 10 of $S U(5)^{\prime}$ undergoes non-trivial "twists" in going around some (or all) of the three directions in $T^{3}$. When this is the case, the minimum eigenvalue of $\mathcal{D}_{T}$ is of order $1 / \alpha$. This is simply because for a generic flat gauge field on the $T^{3}$-fiber there will be no zero mode.

A zero mode of $\mathcal{D}_{T}$ above some point $P \in W$ arises precisely if for some vector in the 10, the monodromies in the fiber are all trivial.

This means that the monodromies lie in the subgroup of $S U(5)^{\prime}$ that leaves fixed that vector. If we represent the $\mathbf{1 0}$ by an antisymmetric $5 \times 5$ matrix $B^{i j}, i, j=1, \ldots, 5$, then the monodromy-invariant vector corresponds to an antisymmetric matrix $B$ that has some nonzero matrix element, say $B^{12}$; the subgroup of $S U(5)^{\prime}$ that leaves $B$ invariant is clearly then a subgroup of $S U(2) \times S U(3)$ (where in these coordinates, $S U(2)$ acts on $i, j=1,2$ and $S U(3)$ on $i, j=3,4,5$ ). Let us consider the case (which we will soon show to be generic) that $B^{12}$ is the only nonzero matrix element of $B$. If so, the subgroup of $S U(5)^{\prime}$ that leaves $B$ fixed is precisely $S U(2) \times S U(3)$. There is actually a distinguished basis in this problem - the one that diagonalizes the monodromies near $P$ - and it is in this basis that $B$ has only one nonzero matrix element.

The commutant of $S U(2) \times S U(3)$ in $E_{8}$ is $S U(6)$. So over the point $P$, the monodromies commute not just with $S U(5)$ but with $S U(6)$. Everything of interest will happen inside this $S U(6)$. The reason for this is that the monodromies at $P$ give large masses to all $E_{8}$ modes except those in the adjoint of $S U(6)$. So we will formulate the rest of the discussion as if the heterotic string gauge group were just $S U(6)$, rather than $E_{8}$. Away from $P$, the monodromies break $S U(6)$ to $S U(5) \times U(1)$ (the global 
structure is actually $U(5))$. Restricting the discussion from $E_{8}$ to $S U(6)$ will mean treating the vacuum gauge bundle as a $U(1)$ bundle (the $U(1)$ being the second factor in $S U(5) \times U(1) \subset S U(6))$ rather than an $S U(5)^{\prime}$ bundle.

The fact that, over $P$, the heterotic string has unbroken $S U(6)$ means that, in the $M$ theory description, the fiber over $P$ has an $S U(6)$ singularity. Likewise, the fact that away from $P$, the heterotic string has only $S U(5) \times U(1)$ unbroken means that the generic fiber, in the $M$ theory description, must contain an $S U(5)$ singularity only, rather than an $S U(6)$ singularity. As for the unbroken $U(1)$, in the $M$ theory description it must be carried by the $C$-field. Indeed, over generic points on $W$ there is a non-zero size $S^{2}$ which shrinks to zero size at $P$ in order that the gauge symmetry at that point increases. Kaluza-Klein reducing $C$ along this $S^{2}$ gives a $U(1)$.

If we move away from the point $P$ in the base, the vector $B$ in the $\mathbf{1 0}$ of $S U(5)^{\prime}$ is no longer invariant under the monodromies. Under parallel transport around the three directions in $T^{3}$, it is transformed by phases $e^{2 \pi i \theta_{j}}, j=1,2,3$. Thus, the three $\theta_{j}$ must all vanish to make $B$ invariant. As $W$ is three-dimensional, we should expect generically that the point $P$ above which the monodromies are trivial is isolated. (Now we can see why it is natural to consider the case that, in the basis given by the monodromies near $P$, only one matrix element of $B$ is nonzero. Otherwise, the monodromies could act separately on the different matrix elements, and it would be necessary to adjust more than three parameters to make $B$ invariant. This would be a less generic situation.) We will only consider the (presumably generic) case that $P$ is disjoint from the singularities of the fibration $Z \rightarrow W$. Thus, the $T^{3}$ fiber over $P$ is smooth (as we have implicitly assumed in introducing the monodromies on $T^{3}$ ).

In [10] we explicitly solved the Dirac equation in a local model for this situation. We found that the net number of chiral zero modes was one. We will not have time to describe the details of the solution here.

In summary, before we translate into the $M$ theory language, the chiral fermions in the heterotic string theory on $Z$ are localised at points on $W$ over which the Wilson lines in the $T^{3}$-fibers are trivial. In $M$ theory this translates into the statement that the chiral fermions are localised at points in $W$ over which the ADE-singularity "worsens". This is also consistent with what we found in the previous section.

\section{M theory Description}

So we have found a local structure in the heterotic string that gives a net chirality the number of massless left-handed 5's minus right-handed 5's - of one. Let us see in more detail what it corresponds to in terms of $M$ theory on a manifold of $G_{2}$ holonomy.

Here it may help to review the case considered in [75] where the goal was geomet- 
ric engineering of charged matter on a Calabi-Yau threefold in Type IIA. What was considered there was a Calabi-Yau three-fold $R$, fibered by $K 3$ 's with a base $W^{\prime}$, such that over a distinguished point $P \in W^{\prime}$ there is a singularity of type $\hat{G}$, and over the generic point in $W^{\prime}$ this singularity is replaced by one of type $G$ - the rank of $\hat{G}$ being one greater than that of $G$. In our example, $\hat{G}=S U(6)$ and $G=S U(5)$. In the application to Type IIA, although $R$ also has a Kahler metric, the focus is on the complex structure. For $\hat{G}=S U(6), G=S U(5)$, let us describe the complex structure of $R$ near the singularities. The $S U(6)$ singularity is described by an equation $x y=z^{6}$ - cf section 5.1. Its "unfolding" depends on five complex parameters and can be written $z y=z^{6}+P_{4}(z)$, where $P_{4}(z)$ is a quartic polynomial in $z$. If - as in the present problem - we want to deform the $S U(6)$ singularity while maintaining an $S U(5)$ singularity, then we must pick $P_{4}$ so that the polynomial $z^{6}+P_{4}$ has a fifth order root. This determines the deformation to be

$$
x y=(z+5 \epsilon)(z-\epsilon)^{5}
$$

where we interpret $\epsilon$ as a complex parameter on the base $W^{\prime}$. Thus, the above equation gives the complex structure of the total space $R$.

What is described above is the partial unfolding of the $S U(6)$ singularity, keeping an $S U(5)$ singularity. In our $G_{2}$ problem, we need a similar construction, but we must view the $S U(6)$ singularity as a hyper-Kahler manifold, not just a complex manifold. In unfolding the $S U(6)$ singularity as a hyper-Kahler manifold, each complex parameter in $P_{4}$ is accompanied by a real parameter that controls the area of an exceptional divisor in the resolution/deformation of the singularity. The parameters are thus not five complex parameters but five triplets of real parameters. (There is an $S O(3)$ symmetry that rotates each triplet. This is the $S O(3)$ rotating the three kahler forms in section 5.1.)

To get a $G_{2}$-manifold, we must combine the complex parameter seen in with a corresponding real parameter. Altogether, this will give a three-parameter family of deformations of the $S U(6)$ singularity (understood as a hyper-Kahler manifold) to a hyper-Kahler manifold with an $S U(5)$ singularity. The parameter space of this deformation is what we have called $W$, and the total space is a seven-manifold that is our desired singular $G_{2}$-manifold $X$, with a singularity that produces the chiral fermions that we analyzed above in the heterotic string language.

To find the hyper-Kahler unfolding of the $S U(6)$ singularity that preserves an $S U(5)$ singularity is not difficult, using Kronheimer's description of the general unfolding via a hyper-Kahler quotient [63] At this stage, we might as well generalize to $S U(N)$, so we consider a hyper-Kahler unfolding of the $S U(N+1)$ singularity to give an $S U(N)$ singularity. The unfolding of the $S U(N+1)$ singularity is obtained by taking a system 
of $N+1$ hypermultiplets $\Phi_{0}, \Phi_{1}, \ldots \Phi_{N}$ with an action of $K=U(1)^{N}$. Under the $i^{t h}$ $U(1)$ for $i=1, \ldots, N, \Phi_{i}$ has charge $1, \Phi_{i-1}$ has charge -1 , and the others are neutral. This configuration of hypermultiplets and gauge fields is known as the quiver diagram of $S U(N+1)$ and appears in studying $D$-branes near the $S U(N+1)$ singularity We let

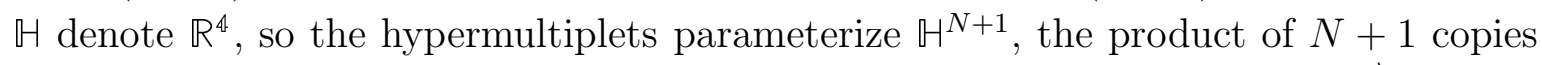
of $\mathbb{R}^{4}$. The hyper-Kahler quotient of $\uplus^{N+1}$ by $K$ is obtained by setting the $\vec{D}$-field (or components of the hyper-Kahler moment map) to zero and dividing by $K$. It is denoted $\mathbb{H}^{N+1} / / K$, and is isomorphic to the $S U(N+1)$ singularity $\mathbb{R}^{4} / \mathbb{Z}_{\mathbb{N}+\mathbb{1}}$. Its unfolding is described by setting the $\vec{D}$-fields equal to arbitrary constants, not necessarily zero. In all, there are $3 N$ parameters in this unfolding - three times the dimension of $K$ - since for each $U(1), \vec{D}$ has three components, rotated by an $S O(3)$ group of $R$-symmetries.

We want a partial unfolding keeping an $S U(N)$ singularity. To describe this, we keep $3(N-1)$ of the parameters equal to zero and let only the remaining three vary;

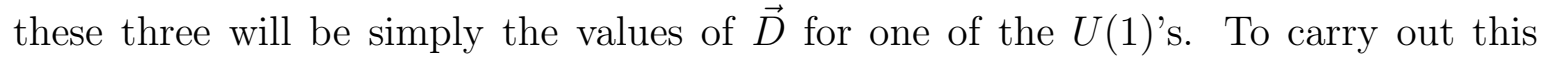
procedure, we first write $K=K^{\prime} \times U(1)^{\prime}$ (where $U(1)^{\prime}$ denotes a chosen $U(1)$ factor of $\left.K=U(1)^{N}\right)$. Then we take the hyper-Kahler quotient of $\uplus^{N+1}$ by $K^{\prime}$ to get a hyperKahler eight-manifold $\hat{X}=\uplus^{N+1} / / K^{\prime}$, after which we take the ordinary quotient, not the hyper-Kahler quotient, by $U(1)^{\prime}$ to get a seven-manifold $X=\hat{X} / U(1)^{\prime}$ that should

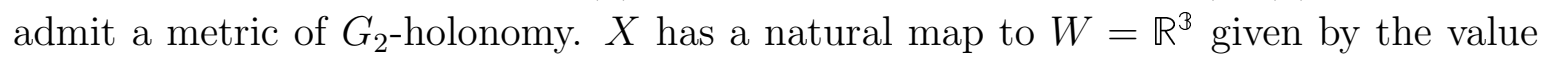
of the $\vec{D}$-field of $U(1)^{\prime}$ - which was not set to zero - and this map gives the fibration of $X$ by hyper-Kahler manifolds.

In the present example, we can easily make this explicit. We take $U(1)^{\prime}$ to be the "last" $U(1)$ in $K=U(1)^{N}$, so $U(1)^{\prime}$ only acts on $\Phi_{N-1}$ and $\Phi_{N}$. $K^{\prime}$ is therefore the product of the first $N-1 U(1)^{\prime}$ 's; it acts trivially on $\Phi_{N}$, and acts on $\Phi_{0}, \ldots, \Phi_{N-1}$ according to the standard quiver diagram of $S U(N)$. So the hyper-Kahler quotient $\mathbb{H}^{N+1} / / K^{\prime}$ is just $\left(\mathbb{H}^{N} / / K^{\prime}\right) \times \mathbb{H}^{\prime}$, where $\mathbb{H}^{N} / / K^{\prime}$ is the $S U(N)$ singularity, isomorphic to $\mathbb{H} / \mathbb{Z}_{\mathbb{N}}$, and $\mathbb{H}^{\prime}$ is parameterized by $\Phi_{N}$. So finally, $X$ will be $\left(\mathbb{H} / \mathbb{Z}_{\mathbb{N}} \times \mathbb{H}^{\prime}\right) / U(1)^{\prime}$. To make this completely explicit, we just need to identify the group actions on $\mathbb{H}$ and $\mathbb{H}^{\prime}$. If we parameterize $\mathbb{H}$ and $\mathbb{H}^{\prime}$ respectively by pairs of complex variables $(a, b)$ and $\left(a^{\prime}, b^{\prime}\right)$ then the $\mathbb{Z}_{\mathbb{N}}$ action on $\mathbb{H}$, such that the quotient $\mathbb{H} / \mathbb{Z}_{\mathbb{N}}$ is the $S U(N)$ singularity, is given by

$$
\left(\begin{array}{l}
a \\
b
\end{array}\right) \rightarrow\left(\begin{array}{c}
e^{2 \pi i k / N} a \\
e^{-2 \pi i k / N} b
\end{array}\right)
$$

while the $U(1)^{\prime}$ action that commutes with this (and preserves the hyper-Kahler structure) is

$$
\left(\begin{array}{l}
a \\
b
\end{array}\right) \rightarrow\left(\begin{array}{c}
e^{i \psi / N} a \\
e^{-i \psi / N} b
\end{array}\right)
$$


The $U(1)^{\prime}$ action on $\mathbb{H}^{\prime}$ is similarly

$$
\left(\begin{array}{c}
a^{\prime} \\
b^{\prime}
\end{array}\right) \rightarrow\left(\begin{array}{c}
e^{i \psi / N} a^{\prime} \\
e^{-i \psi / N} b^{\prime}
\end{array}\right)
$$

In all, if we set $\lambda=e^{i \psi / N}, w_{1}=\bar{a}^{\prime}, w_{2}=b^{\prime}, w_{3}=a, w_{4}=\bar{b}$, then the quotient $\left(\mathbb{H} / \mathbb{Z}_{\mathbb{N}} \times \mathbb{H}^{\prime}\right) / U(1)$ can be described with four complex variables $w_{1}, \ldots, w_{4}$ modulo the equivalence

$$
\left(w_{1}, w_{2}, w_{3}, w_{4}\right) \rightarrow\left(\lambda^{N} w_{1}, \lambda^{N} w_{2}, \lambda w_{3}, \lambda w_{4}\right), \quad|\lambda|=1
$$

This quotient is a cone on a weighted projective space $\mathbb{W} \mathbb{C} \mathbf{P}_{N, N, 1,1}^{3}$. In fact, if we impose the above equivalence relation for all nonzero complex $\lambda$, we would get the weighted projective space itself; by imposing this relation only for $|\lambda|=1$, we get a cone on the weighted projective space. Note, that the conical metric of $G_{2}$-holonomy on this space does not use usual Kahler metric on weighted projective space.

$\mathbb{W} \mathbb{C} \mathbf{P}_{N, N, 1,1}^{3}$ has a family of $\mathrm{A}_{N-1}$-singularities at points $\left(w_{1}, w_{2}, 0,0\right)$. This is easily seen by setting $\lambda$ to $e^{2 \pi i / N}$. This set of points is a copy of $\mathbb{C} \mathbf{P}^{1}=\mathbf{S}^{2}$. Our proposed $G_{2^{-}}$ manifold is a cone over weighted projective space, so it has a family of $A_{N-1}$-singularities which are a cone over this $\mathbf{S}^{\mathbf{2}}$. This is of course a copy of $\mathbb{R}^{\mathbb{B}}$. Away from the origin in $\mathbb{R}^{\mathbb{B}}$ the only singularities are these orbifold singularities. At the origin however, the whole manifold develops a conical singularity. There, the 2 -sphere, which is incontractible in the bulk of the manifold, shrinks to zero size. This is in keeping with the anomaly inflow arguments of the previous section. There we learned that an ADE-singularity which worsens over a point in $W$ is a good candidate for the appearance of chiral fermions. Here, via duality with the heterotic string, we find that the conical singularity in this example supports one chiral fermion in the $\mathbf{N}$ of the $S U(N)$ gauge symmetry coming from the $A_{N-1}$-singularity. In fact, the $U(1)$ gauge symmetry from the $C$-field in this example, combines with the $S U(N)$ to give a gauge group which is globally $U(N)$ and the fermion is in the fundamental representation.

Some extensions of this can be worked out in a similar fashion. Consider the case that away from $P$, the monodromies break $S U(N+1)$ to $S U(p) \times S U(q) \times U(1)$, where $p+q=N+1$. Analysis of the Dirac equation along the above lines shows that such a model will give chiral fermions transforming as $(\mathbf{p}, \overline{\mathbf{q}})$ under $S U(p) \times S U(q)$ (and charged under the $U(1))$. To describe a dual in $M$ theory on a manifold of $G_{2}$ holonomy, we let $K=K^{\prime} \times U(1)^{\prime}$, where now $K^{\prime}=K_{1} \times K_{2}, K_{1}$ being the product of the first $p-1 U(1)$ 's in $K$ and $K_{2}$ the product of the last $q-1$, while $U(1)^{\prime}$ is the $p^{\text {th }} U(1)$. Now we must define $\hat{X}=\uplus^{N+1} / / K^{\prime}$, and the manifold admitting a metric of $G_{2}$ holonomy should be $\hat{X} / U(1)^{\prime}$.

We can compute $\hat{X}$ easily, since $K_{1}$ acts only on $\Phi_{1}, \ldots, \Phi_{p}$ and $K_{2}$ only on $\Phi_{p+1}, \ldots, \Phi_{N+1}$. The hyper-Kahler quotients by $K_{1}$ and $K_{2}$ thus simply construct the $S U(p)$ and $S U(q)$ 
singularities, and hence $\hat{X}=\mathbb{H} / \mathbb{Z}_{\mathbb{p}} \times \mathbb{H} / \mathbb{Z}_{\mathbb{q}}$. $\hat{X}$ has planes of $\mathbb{Z}_{\mathbb{p}}$ and $\mathbb{Z}_{q}$ singularities, which will persist in $X=\hat{X} / U(1)^{\prime}$, which will also have a more severe singularity at the origin. So the model describes a theory with $S U(p) \times S U(q)$ gauge theory and chiral fermions supported at the origin. $U(1)^{\prime}$ acts on $\mathbb{H} / \mathbb{Z}_{\mathbb{p}}$ and $\mathbb{H} / \mathbb{Z}_{\mathbb{q}}$ as the familiar global symmetry that preserves the hyper-Kahler structure of the $S U(p)$ and $S U(q)$ singularities. Representing those singularities by pairs $(a, b)$ and $\left(a^{\prime}, b^{\prime}\right)$ modulo the usual action of $\mathbb{Z}_{\mathbb{p}}$ and $\mathbb{Z}_{\mathbb{q}}, U(1)^{\prime}$ acts by

$$
\left(\begin{array}{c}
a \\
b
\end{array}\right) \rightarrow\left(\begin{array}{c}
e^{i \psi / p} a \\
e^{-i \psi / p} b
\end{array}\right) \text { and }\left(\begin{array}{c}
a^{\prime} \\
b^{\prime}
\end{array}\right) \rightarrow\left(\begin{array}{c}
e^{-i \psi / q} a^{\prime} \\
e^{i \psi / q} b^{\prime}
\end{array}\right)
$$

Now if $p$ and $q$ are relatively prime, we set $\lambda=e^{i \psi / p q}$, and we find that the $U(1)^{\prime}$ action on the complex coordinates $w_{1}, \ldots, w_{4}$ (which are defined in terms of $a, b, a^{\prime}, b^{\prime}$ by the same formulas as before) is

$$
\left(w_{1}, w_{2}, w_{3}, w_{4}\right) \rightarrow\left(\lambda^{p} w_{1}, \lambda^{p} w_{2}, \lambda^{q} w_{3}, \lambda^{q} w_{4}\right) .
$$

If $p$ and $q$ are relatively prime, then the $U(1)^{\prime}$ action, upon taking $\lambda$ to be a $p^{\text {th }}$ or $q^{\text {th }}$ root of 1 , generates the $\mathbb{Z}_{\mathbb{P}} \times \mathbb{Z}_{\mathbb{Q}}$ orbifolding that is part of the original definition of $\hat{X}$. Hence in forming the quotient $\hat{X} / U(1)^{\prime}$, we need only to act on the $w$ 's by the equivalence relation. The quotient is therefore a cone on a weighted projective space $\mathbb{W} C \mathbf{P}_{p, p, q, q}^{3}$. If $p$ and $q$ are not relatively prime, we let $(p, q)=r(n, m)$ where $r$ is the greatest common divisor and $n$ and $m$ are relatively prime. Then we let $\lambda=\exp (i r \psi / p q)$, so the equivalence relation above is replaced with

$$
\left(w_{1}, w_{2}, w_{3}, w_{4}\right) \rightarrow\left(\lambda^{n} w_{1}, \lambda^{n} w_{2}, \lambda^{m} w_{3}, \lambda^{m} w_{4}\right)
$$

To reproduce $\hat{X} / U(1)$ we must now also divide by $\mathbb{Z}_{\mathbb{\varpi}}$, acting by

$$
\left(w_{1}, w_{2}, w_{3}, w_{4}\right) \rightarrow\left(\zeta w_{1}, \zeta w_{2}, w_{3}, w_{4}\right),
$$

where $\zeta^{r}=1$. So $X$ is a cone on $\mathbb{W} \mathbb{C} \mathbf{P}_{n, n, m, m}^{3} / \mathbb{Z}_{r}$.

\subsection{Other Gauge Groups and Matter Representations}

We now explain how to generalise the above construction to obtain singularities with more general gauge groups and chiral fermion representations. Suppose that we want to get chiral fermions in the representation $R$ of a simply-laced group $G$. This can be achieved for certain representations. We find a simply-laced group $\hat{G}$ of rank one more than the rank of $G$, such that $\hat{G}$ contains $G \times U(1)$ and the Lie algebra of $\hat{G}$ decomposes as $\mathbf{g} \oplus \mathbf{o} \oplus \mathbf{r} \oplus \overline{\mathbf{r}}$, where $\mathbf{g}$ and $\mathbf{o}$ are the Lie algebras of $G$ and $U(1), \mathbf{r}$ transforms as 
$R$ under $G$ and of charge 1 under $U(1)$, and $\overline{\mathbf{r}}$ transforms as the complex conjugate. Such a $\hat{G}$ exists only for special $R$ 's, and these are the $R$ 's that we will generate from $G_{2}$ singularities.

Given $\hat{G}$, we proceed as above on the heterotic string side. We consider a family of $\mathbf{T}^{3}$ 's, parameterized by $W$, with monodromy that at a special point $P \in W$ leaves unbroken $\hat{G}$, and at a generic point breaks $\hat{G}$ to $G \times U(1)$. We moreover assume that near $P$, the monodromies have the same sort of generic behavior assumed above. Then the same computation as above will show that the heterotic string has, in this situation, a single multiplet of fermion zero modes (the actual chirality depends on the solving the Dirac equation) in the representation $R$, with $U(1)$ charge 1.

Dualizing this to an $M$ theory description, over $P$ we want a $\hat{G}$ singularity, while over a generic point in $W$, we should have a $G$ singularity. Thus, we want to consider the unfolding of the $\hat{G}$ singularity (as a hyper-Kahler manifold) that preserves a $G$ singularity. To do this is quite simple. We start with the Dynkin diagram of $\hat{G}$. The vertices are labeled with integers $n_{i}$, the Dynkin indices. In Kronheimer's construction, the $\hat{G}$ singularity is obtained as the hyper-Kahler quotient of $\mathbb{H}^{k}$ (for some $k$ ) by the action of a group $K=\prod_{i} U\left(n_{i}\right)$. Its unfolding is obtained by allowing the $\vec{D}$-fields of the $U(1)$ factors (the centers of the $U\left(n_{i}\right)$ ) to vary.

The $G$ Dynkin diagram is obtained from that of $\hat{G}$ by omitting one node, corresponding to one of the $U\left(n_{i}\right)$ groups; we write the center of this group as $U(1)^{\prime}$. Then we write $K$ (locally) as $K=K^{\prime} \times U(1)^{\prime}$, where $K^{\prime}$ is defined by replacing the relevant $U\left(n_{i}\right)$ by $S U\left(n_{i}\right)$. We get a hyper-Kahler eight-manifold as the hyper-Kahler quotient $\hat{X}=\mathbb{H}^{k} / / K^{\prime}$, and then we get a seven-manifold $X$ by taking the ordinary quotient $X=\hat{X} / U(1)^{\prime}$. This maps to $W=\mathbb{R}^{\mathbb{B}}$ by taking the value of the $U(1)^{\prime} \vec{D}$-field, which was not set to zero. The fiber over the origin is obtained by setting this $\vec{D}$-field to zero after all, and gives the original $\hat{G}$ singularity, while the generic fiber has a singularity of type $G$.

One can readily work out examples of pairs $G, \hat{G}$. We will just consider the cases most relevant for grand unification. For $G=S U(N)$, to get chiral fields in the antisymmetric tensor representation, $\hat{G}$ should be $S O(2 N)$. For $G=S O(10)$, to get chiral fields in the 16, $\hat{G}$ should be $E_{6}$. For $G=S O(2 k)$, to get chiral fields in the $\mathbf{2 k}, \hat{G}$ should be $S O(2 k+2)$. (Note in this case that $\mathbf{2} \mathbf{k}$ is a real representation. However, the monodromies in the above construction break $S O(2 k+2)$ to $S O(2 k) \times U(1)$, and the massless $\mathbf{2 k}$ 's obtained from the construction are charged under the $U(1)$; under $S O(2 k) \times U(1)$ the representation is complex.) For $2 k=10$, this example might be used in constructing $S O(10)$ GUT's. For $G=E_{6}$, to get 27's, $\hat{G}$ should be $E_{7}$. A useful way to describe the topology of $X$ in these examples is not clear.

In this construction, we emphasized, on the heterotic string side, the most generic 
special monodromies that give enhanced gauge symmetry, which corresponds on the $M$ theory side to omitting from the hyper-Kahler quotient a rather special $U(1)$ that is related to a single node of the Dynkin diagram. We could also consider more general heterotic string monodromies; this would correspond in $M$ theory to omitting a more general linear combination of the $U(1)$ 's. For a more detailed discussion of some of these examples see [72]. 


\section{Topology Change in $M$ theory on Exceptional Holonomy Manifolds}

\subsection{Topology Change in $M$ theory}

In this section we discuss topology changing transitions, by which we mean a particular behavior of the manifold $X$ (and the associated physics) in the singular limit when one can go to a space with a different topology. In Calabi-Yau manifolds many examples of such transitions are known and can be understood using conformal field theory methods, see e.g. [77] and references therein. Some of these transitions give rise to analogous topology changing transitions in $G_{2}$ and $\operatorname{Spin}(7)$ manifolds obtained from finite quotients of Calabi-Yau spaces that we discussed in section 3.1. In the context of compact manifolds with $G_{2}$ holonomy this was discussed in [17, 78, 79]. One typically finds a transition between different topologies of $X$, such that the following sum of the Betti numbers remains invariant

$$
b_{2}+b_{3}=\text { const }
$$

which is precisely what one would expect from the conformal field theory considerations [55]. One can interpret the condition (7.121) as a feature of the mirror phenomenon for $G_{2}$ manifolds [55, 80, 81, 27].

Here, we shall discuss topology change in the non-compact asymptotically conical $G_{2}$ and $\operatorname{Spin}(7)$ manifolds of section 3.2, which are in a sense the most basic examples of singularities that reveal features particular to exceptional holonomy. Notice, that all of these manifolds have the form:

$$
X \cong B \times(\text { contractible })
$$

where $B$ is a non-trivial cycle (a bolt), e.g. $B=\mathbf{S}^{3}, \mathbf{S}^{4}, \mathbb{C P}^{2}$, or something else. Therefore, it is natural to ask:

$$
\text { "What happens if } \operatorname{Vol}(B) \rightarrow 0 \text { ?" }
$$

In this limit the geometry becomes singular and, as we discussed earlier, there are many possibilities for $M$ theory dynamics associated with it. One possibility is a topology change, which is indeed what we shall find in some of the cases below.

Although we are mostly interested in exceptional holonomy manifolds, it is instructive to start with topology changing transitions in Calabi-Yau manifolds, where one finds two prototypical examples: 


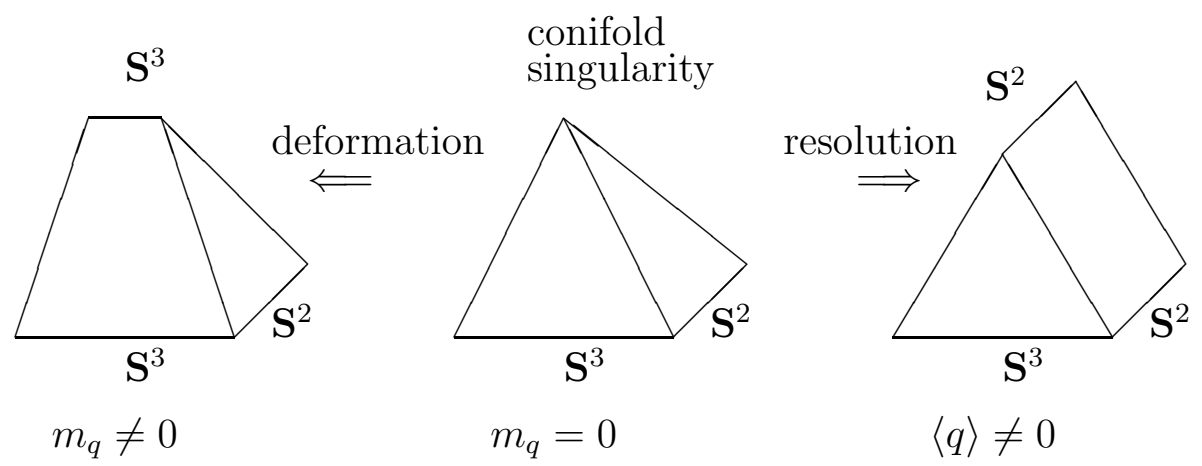

Figure 7: Conifold transition in type IIB string theory.

The Flop is a transition between two geometries, where one two-cycle shrinks to a point and a (topologically) different two-cycle grows. This process can be schematically described by the diagram:

$$
\mathbf{S}_{(1)}^{2} \longrightarrow \cdot \mathbf{S}_{(2)}^{2}
$$

This transition is smooth in string theory [82, 83].

The Conifold transition is another type of topology change, in which a three-cycle shrinks and is replaced by a two-cycle:

$$
\mathbf{S}^{3} \longrightarrow \cdot \mathbf{S}^{2}
$$

Ulike the flop, it is a real phase transition in the low-energy dynamics which can be understood as the condensation of massless black holes [84, 85]. Let us briefly recall the main arguments.

As the name indicates, the conifold is a cone over a five dimensional base space. The base has topology $\mathbf{S}^{2} \times \mathbf{S}^{3}$ (see Figure 7 ). Two different ways to desingularize this space - called the deformation and the resolution — correspond to replacing the singularity by a finite size $\mathbf{S}^{3}$ or $\mathbf{S}^{2}$, respectively. Thus, we have two different spaces, with topology $\mathbf{S}^{3} \times \mathbb{R}^{3}$ and $\mathbf{S}^{2} \times \mathbb{R}^{4}$, which asymptotically look the same.

In type IIB string theory, the two phases of the conifold geometry correspond to different branches in the four-dimensional $\mathcal{N}=2$ low-energy effective field theory. In the deformed conifold phase, D3-branes wrapped around the 3-sphere give rise to a low-energy field $q$, with mass determined by the size of the $\mathbf{S}^{3}$. In the effective four-dimensional supergravity theory these states appear as heavy, point-like, extremal black holes. On the other hand, in the resolved conifold phase the field $q$ acquires an expectation value reflecting the condensation of these black holes. Of course, in order to make the transition from one phase to the other, the field $q$ must become massless somewhere and this happens at the conifold singularity, as illustrated in Figure 7.

Now, let us proceed to topology change in $G_{2}$ manifolds. Here, again, one finds 


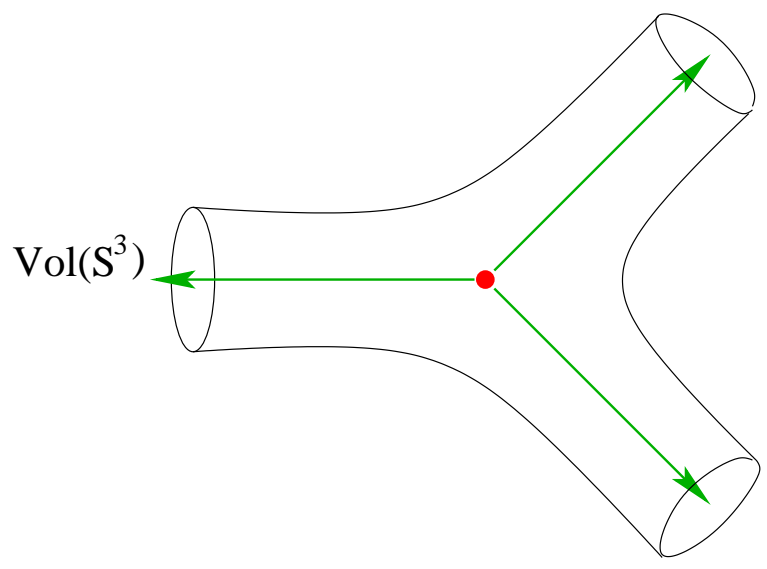

Figure 8: Quantum moduli space of $M$ theory on $G_{2}$ manifold $X$ with topology $\mathbf{S}^{3} \times \mathbb{R}^{4}$. Green lines represent the 'geometric moduli space' parametrized by the volume of the $\mathbf{S}^{3}$ cycle, which is enlarged to a smooth complex curve by taking into account $C$-field and quantum effects. The resulting moduli space has three classical limits, which can be connected without passing through the point where geometry becomes singular (represented by red dot in this picture).

two kinds of topology changing transitions, which resemble the flop and the conifold transitions in Calabi-Yau manifolds:

The $G_{2}$ Flop is a transition where a 3 -cycle collapses and gets replaced by a (topologically) distinct 3-cycle:

$$
\mathbf{S}_{(1)}^{3} \longrightarrow \cdot \mathbf{S}_{(2)}^{3}
$$

Note, that this is indeed very similar to the flop transition in Calabi-Yau manifolds, where instead of a 2-cycle we have a 3-cycle shrinking. The physics is also similar, with membranes playing the role of string world-sheet instantons. Remember, that the latter were crucial for the flop transition to be smooth in string theory. For a very similar reason, the $G_{2}$ flop transition is smooth in $M$ theory. This was first prpoposed by Atiyah, Maldacena, and Vafa [7] (see also Acharya [6]), for a 7-manifold with topology

$$
X \cong \mathbf{S}^{3} \times \mathbb{R}^{4}
$$

and studied further by Atiyah and Witten [8]. In particular, they found that $M$ theory on $X$ has three classical branches, related by a triality permutation symmetry, so that the quantum moduli space looks as shown on Figure 8. Once again, the important point is that there is no singularity in quantum theory.

If we denote the space $X$ on the $i$-th branch as $X_{i}$, then each $X_{i}$ can be constructed as a cone over $Y=\mathbf{S}^{3} \times \mathbf{S}^{3}$ described in the example of section 3.2. Namely, as in (3.34), 


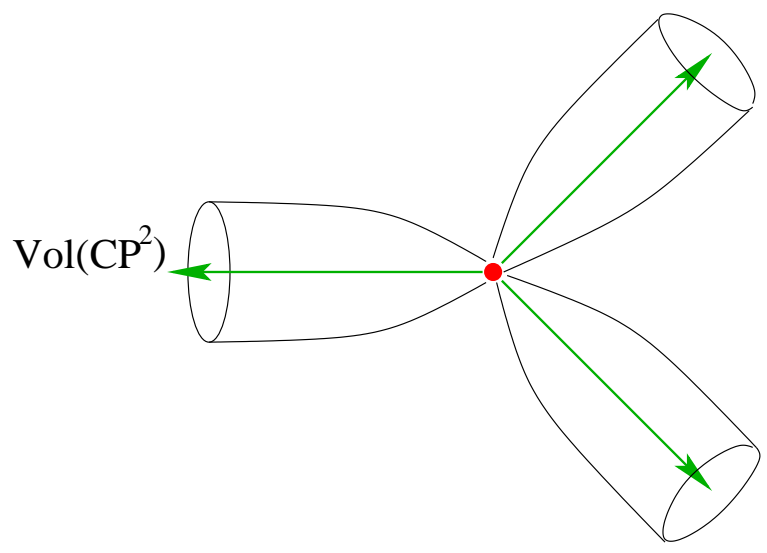

Figure 9: Quantum moduli space of $M$ theory on $G_{2}$ manifold $X$ with topology $\mathbb{C P}^{2} \times$ $\mathbb{R}^{3}$. Green lines represent the 'geometric moduli space' parametrized by the volume of the $\mathbb{C} \mathbf{P}^{2}$ cycle, which is enlarged to a singular complex curve by taking into account $C$-field and quantum effects. The resulting moduli space has three classical limits. In order to go from one branch to another one necessarily has to pass through the point where geometry becomes singular (represented by red dot in this picture).

we can view $Y$ as a homogeneous space $G / K$, where $G=S U(2)^{3}$ and $K=S U(2)$ is its diagonal subgroup. This description is particularly convenient because it makes all the symmetries of $M$ theory on $X_{i}$ very explicit and easy to see. Specifically, the space $Y$ can be described in terms of three $S U(2)$ elements, $\left(g_{1}, g_{2}, g_{3}\right) \in G$, with the following equivalence relation

$$
\left(g_{1}, g_{2}, g_{3}\right)=\left(g_{1} h, g_{2} h, g_{3} h\right)
$$

Then, the seven-manifold $X_{i}$ is obtained by "filling in" the $i$-th copy of $S U(2)$. From construction, it is clear that the resulting manifolds $X_{i}$ are permuted by the triality symmetry, and have the topology (7.122). Similar to (7.123), the homology of $Y$ is generated by three 3 -cycles $D_{i}$, subject to a linear relation

$$
D_{1}+D_{2}+D_{3}=0
$$

where the 3 -cycle $D_{i}$ is obtained by projecting the $i$-th copy of $S U(2)$ in $Y=S U(2)^{3} / S U(2)$. The homology of $X_{i}$ is obtained by imposing a further condition, $D_{i}=0$, which indicates that the $i$-th copy of $S U(2)$ is contractible in $X$. Therefore, on the $i$-th branch we have $D_{i}=0$ and $D_{i+1}=-D_{i-1}$, where $i \in \mathbb{Z} \bmod 3$.

Let us proceed to another kind of topology changing transition in manifolds with $G_{2}$ holonomy.

A Phase Transition can be found in $M$ theory on a $G_{2}$ manifold with topology

$$
X \cong \mathbb{C} \mathbf{P}^{2} \times \mathbb{R}^{3}
$$


A singularity develops when the $\mathbb{C} \mathbf{P}^{2}$ cycle shrinks. As in the conifold transition, the physics of $M$ theory on this space also becomes singular at this point, in a sense that certain parameters jump discontinuously even in quantum theory. Hence, this is a genuine phase transition [8]. Note, however, that unlike the conifold transition in type IIB string theory, this phase transition is not associated with condensation of any particle-like states in $M$ theory ${ }^{12}$ on $X$. Indeed, there are no 4-branes in $M$ theory, which could result in particle-like objects by wrapping around the collapsing $\mathbb{C} \mathbf{P}^{2}$ cycle.

Like the $G_{2}$ flop transition, this phase transition has three classical branches, which are related by triality symmetry, see Figure 9 . The important difference, of course, is that now one can go from one branch to another only through the singular point. In this transition one $\mathbb{C} \mathbf{P}^{2}$ cycle shrinks and another (topologically different) $\mathbb{C} \mathbf{P}^{2}$ cycle grows:

$$
\mathbb{C} \mathbf{P}_{(1)}^{2} \longrightarrow \cdot \longrightarrow \mathbb{C} \mathbf{P}_{(2)}^{2}
$$

Moreover, the global symmetry of the theory changes discontinuously as we go through the singular point. Specifically, the singular point is characterised by a global $U(1) \times$ $U(1)$ symmetry, which is broken to different $U(1)$ subgroups on each of the three branches. The generators of these subgroups are permuted by the triality symmetry and add up to zero.

It was proposed in [8], that the effective $\mathcal{N}=1$ physics of $M$ theory on $X$ can be described by a model with three chiral multiplets $\Phi_{i}, i=1,2,3$, with the superpotential

$$
\mathcal{W}=\Phi_{1} \Phi_{2} \Phi_{3}
$$

Extremizing the superpotential $\mathcal{W}$, it is easy to see that the space of vacua in this theory indeed consists of three branches, so that the $i$-th branch is parameterised by the field $\Phi_{i}$. The three branches meet at the origin, $\Phi_{1}=\Phi_{2}=\Phi_{3}=0$, where one finds the global $U(1) \times U(1)$ symmetry, which acts on the chiral fields as $\Phi_{i} \rightarrow e^{i \theta_{i}} \Phi_{i}$ with $\theta_{1}+\theta_{2}+\theta_{3}=0$. On the $i$-th branch this global symmetry is spontaneously broken to a $U(1)$ subgroup, characterized by $\theta_{i}=0$.

One way to see that this is indeed the right physics of $M$ theory on $X$ is to reduce it to type IIA theory with D6-branes in flat space-time [8]. As a result, one finds precisely the configuration of three intersecting D6-branes described in the example of section 5.2. Then, the three branches of $M$ theory on $X$ can be easily identified with three different deformations of the brane configuration shown of Figure 6, and the superpotential (7.125) is the effect of the string world-sheet disk instantons.

Finally, we come to the last and most difficult of the holonomy groups: $\operatorname{Spin}(7)$.

\footnotetext{
${ }^{12}$ However, such interpretation can be given in type IIA string theory [26].
} 


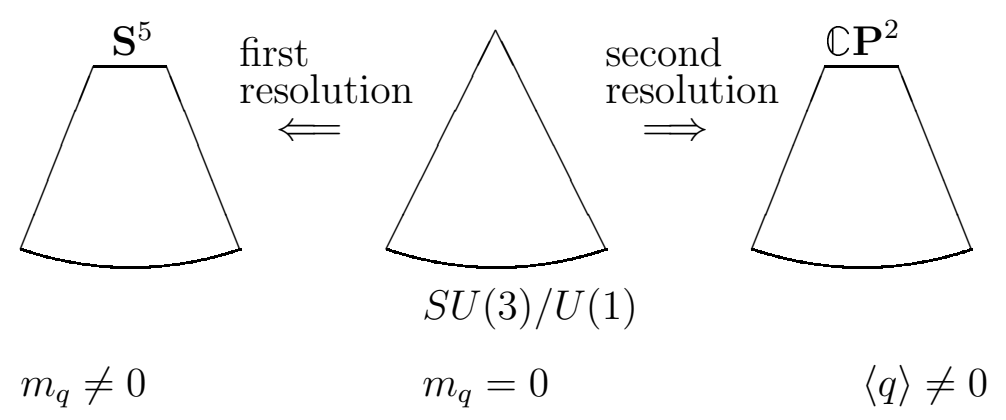

Figure 10: Conifold transition in $M$ theory on a manifold with $\operatorname{Spin}(7)$ holonomy.

The $\operatorname{Spin}(7)$ Conifold is the cone on $S U(3) / U(1)$. It was conjectured in [26] that the effective dynamics of $M$ theory on the $\operatorname{Spin}(7)$ conifold is analogous to that of type IIB string theory on the usual conifold. Namely, the $\operatorname{Spin}(7)$ cone on $S U(3) / U(1)$ has two different desingularizations, obtained by replacing the conical singularity with either a 5-sphere or with a $\mathbb{C} \mathbf{P}^{2}$, see Figure 10. As a result, we obtain two different $\operatorname{Spin}(7)$ manifolds, with topology

$$
\mathbb{C} \mathbf{P}^{2} \times \mathbb{R}^{4} \quad \text { and } \quad \mathbf{S}^{5} \times \mathbb{R}^{3}
$$

which are connected via the topology changing transition

$$
\mathbb{C P}^{2} \longrightarrow \cdot \mathbf{S}^{5}
$$

Like the conifold transition in string theory [84, 85], the $\operatorname{Spin}(7)$ conifold in $M$ theory has a nice interpretation in terms of the condensation of branes. Namely, in the $\mathbf{S}^{5}$ phase we have extra massive states obtained upon quantization of the $M 5$-brane wrapped around the five-sphere. The mass of these states is related to the volume of the $\mathbf{S}^{5}$. At the conifold point where the five-sphere shrinks, these $M 5$-branes become massless as suggested by the classical geometry. At this point, the theory may pass through a phase transition into the Higgs phase, associated with the condensation of these five-brane states, see Figure 10.

To continue the analogy with the Calabi-Yau conifold, recall that the moduli space of type II string theory on the Calabi-Yau conifold has three semi-classical regimes. The deformed conifold provides one of these, while there are two large-volume limits of the resolved conifold, related to each other by a flop transition. In fact, the same picture emerges for the $\operatorname{Spin}(7)$ conifold. In this case, however, the two backgrounds differ not in geometry, but in the $G$-flux. It was shown in [36] that, due to the membrane anomaly of [47], $M$ theory on $X \cong \mathbb{C P}^{2} \times \mathbb{R}^{4}$ is consistent only for half-integral units of $G$-flux through the $\mathbb{C} \mathbf{P}^{2}$ bolt. Namely, after the transition from $X \cong \mathbf{S}^{5} \times \mathbb{R}^{3}$, the $G$-flux may 
take the values $\pm 1 / 2$, with the two possibilities related by a parity transformation [26]. Thus, the moduli space of $M$ theory on the $\operatorname{Spin}(7)$ cone over $S U(3) / U(1)$ also has three semi-classical limits: one with the parity invariant background geometry $\mathbf{S}^{5} \times \mathbb{R}^{3}$, and two with the background geometry $X \cong \mathbb{C P}^{2} \times \mathbb{R}^{4}$ where parity is spontaneously broken, see Figure 11. The last two limits are mapped into each other under the parity transformation.

This picture is reproduced in the effective low-energy theory if we include in the spectrum light states corresponding to M5-branes wrapped over the five-sphere:

Effective Theory: $\mathcal{N}=1, D=3$ Maxwell-Chern-Simons theory with one charged complex scalar multiplet $q$

Here, it is the Higgs field $q$ that appears due to quantizaion of the $M 5$-branes. In this theory, different topological phases correspond to the Coulomb and Higgs branches:

$$
\begin{aligned}
\mathbf{S}^{5} \times \mathbb{R}^{3} & \Longleftrightarrow \quad \text { Coulomb branch } \\
\mathbb{C} \mathbf{P}^{2} \times \mathbb{R}^{4} & \Longleftrightarrow \quad \text { Higgs branch }
\end{aligned}
$$

Further agreement in favor of this identification arises from examining the various extended objects that exist in $M$ theory on $\mathbb{C P}^{2} \times \mathbb{R}^{4}$, obtained from wrapped $M 5$ or M2-branes. For example, we can consider an $M 2$-brane over $\mathbb{C} \mathbf{P}^{1}$ inside $\mathbb{C} \mathbf{P}^{2} \times \mathbb{R}^{4}$. This non-BPS state has a semi-classical mass proportional to the volume of $\mathbb{C} \mathbf{P}^{1}$, and is electrically charged under the global $U(1)_{J}$ symmetry of our gauge theory. Therefore, this state can be naturally identified with a vortex. Note, that this state can be found only in the $\mathbb{C P}^{2}$ phase (i.e. in the Higgs phase), in complete agreement with the low-energy physics.

In view of the interesting phenomena associated to branes in the conifold geometry, and their relationship to the conifold transition [86, 87], it would be interesting to learn more about the $\operatorname{Spin}(7)$ transition using membrane probes in this background, and also to study the corresponding holographic renormalization group flows. For work in this area, see $[88,89,90]$.

\subsection{Relation to Geometric Transition}

In the previous section we described the basic examples of topology changing transitions in exceptional holonomy manifolds, and commented on the important aspects of $M$ theory dynamics in these transitions. As we explain in this section, some of these transitions also have a nice interpretation in type IIA string theory, realizing dualities between backgrounds involving D6-branes and Ramond-Ramond fluxes in manifolds with more restricted holonomy. Specifically, we will consider two cases: 


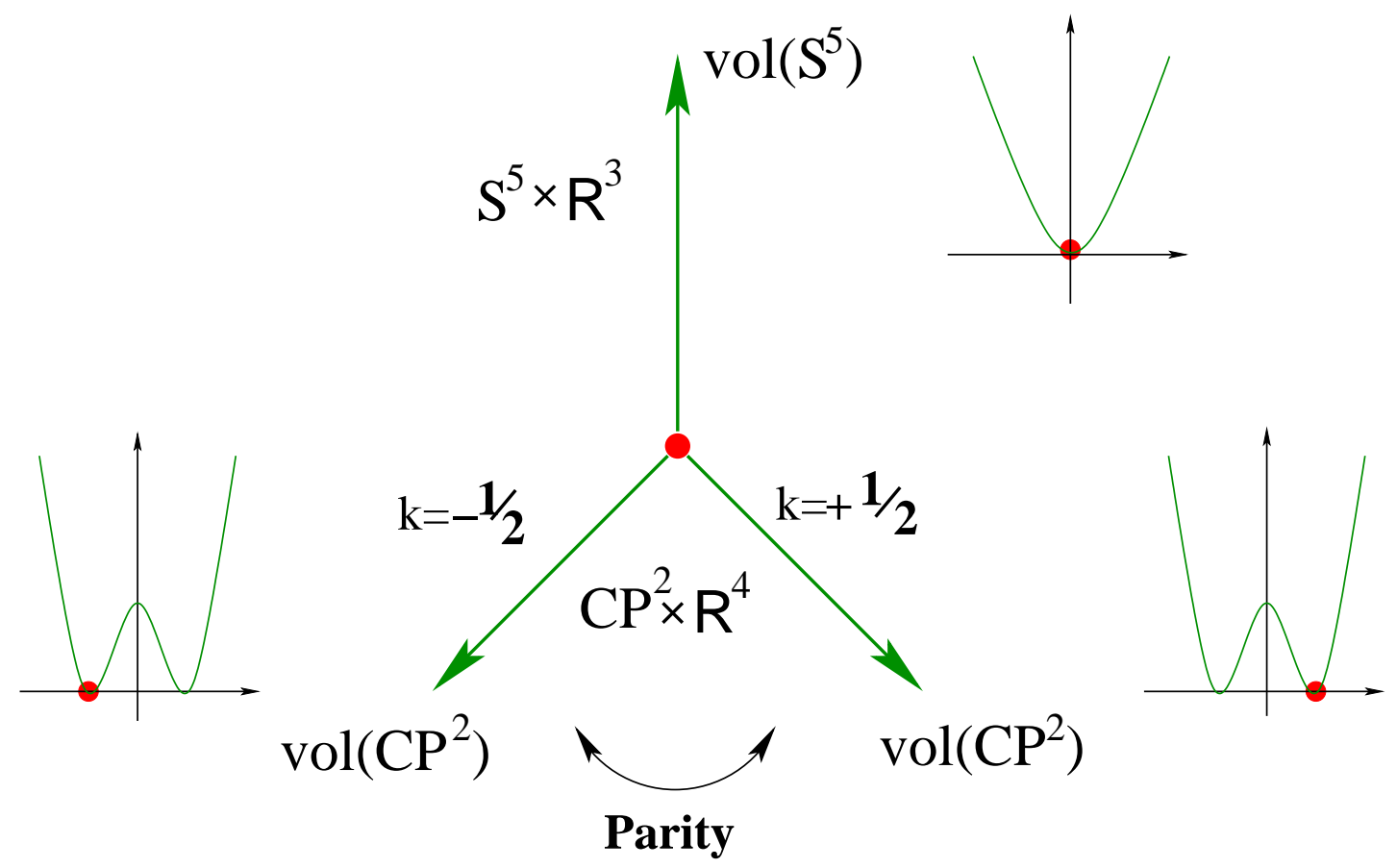

Figure 11: The moduli space of $M$ theory on the $\operatorname{Spin}(7)$ cone over $S U(3) / U(1)$ can be compared to the vacuum structure of a system with spontaneous symmetry breaking. On this picture, the G-flux is measured by $k=\int_{\mathbb{C} \mathbf{P}^{2}} G / 2 \pi$. 


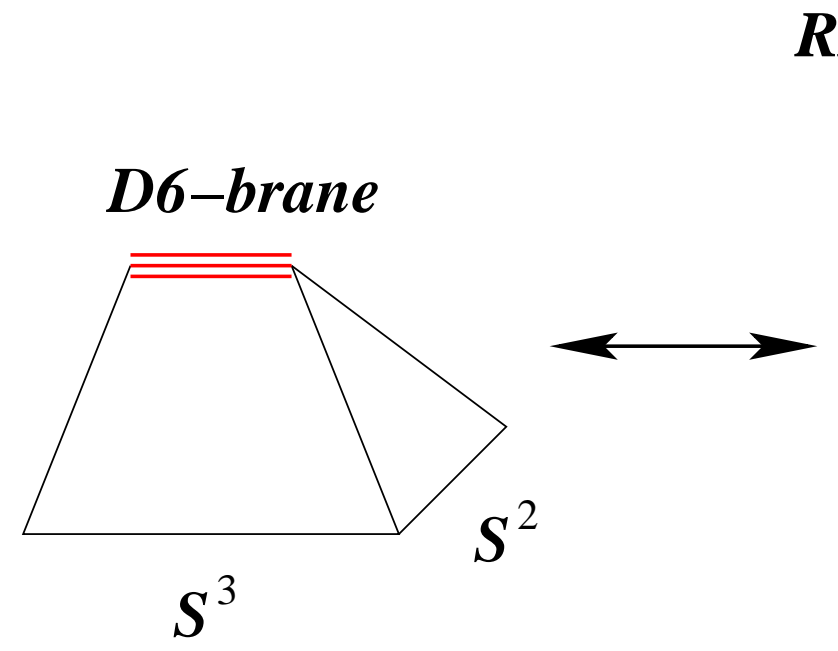

RR flux

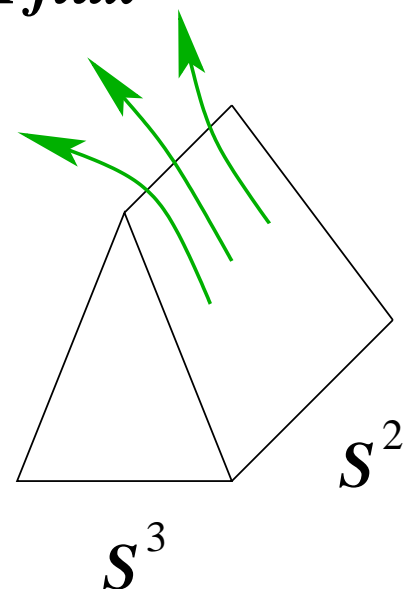

Figure 12: Geometric transition in IIA string theory connecting D6-branes wrapped around $\mathbf{S}^{3}$ in the deformed conifold geometry and resolved conifold with RamondRamond 2-form flux through the $\mathbf{S}^{2}$.

- $\mathbf{S U}(\mathbf{3}) \rightarrow \mathbf{G}_{\mathbf{2}}$ : We start with a relation between the conifold transition in the presence of extra fluxes and branes and the $G_{2}$ flop transition in $M$ theory [6, 7]. Note, that these two transitions are associated with different holonomy groups ${ }^{13}$ and, in particular, with different amount of unbroken supersymmetry. The relation, however, appears when we introduce extra matter fields, represented either by D-branes or by fluxes. They break supersymmetry further, therefore, providing a relation between two different holonomy groups.

In order to explain how this works in the case of the conifold, let us consider type IIA theory on the deformed conifold geometry, $T^{*} \mathbf{S}^{3} \cong \mathbf{S}^{3} \times \mathbb{R}^{3}$. This already breaks supersymmetry down to $\mathcal{N}=2$ in four dimensions. One can break supersymmetry further, to $\mathcal{N}=1$, by wrapping a space-filling D6-brane around the supersymmetric (special Lagrangian) $\mathbf{S}^{3}$ cycle in this geometry. Then, a natural question to ask is: "What happens if one tries to go through the conifold transition with the extra D6brane?". One possibility could be that the other branch is no longer connected and the transition is not possible. However, this is not what happens. Instead the physics is somewhat more interesting. According to [91], the transition proceeds, but now the two branches are smoothly connected, with the wrapped D6-brane replaced by Ramond-Ramond 2-form flux through the $\mathbf{S}^{2}$ cycle of the resolved conifold, see Figure 12 .

\footnotetext{
${ }^{13}$ That is why we refer to this case as $S U(3) \rightarrow G_{2}$.
} 
As we explained in section 5.2, both D6-branes and Ramond-Ramond 2-form tensor fields lift to purely geometric backgrounds in $M$ theory. Therefore, the geometric transition described above should lift to a transition between two purely geometric backgrounds in $M$ theory (hence, the name). Since these geometries must preserve the same amount of supersymmetry, namely $\mathcal{N}=1$ in four dimensions, we conclude that we deal with a $G_{2}$ transition. In fact, it is the familiar flop transition in $M$ theory on a $G_{2}$ manifold $[6,7]$ :

$$
X \cong \mathbf{S}^{3} \times \mathbb{R}^{3}
$$

Indeed, if we start on one of the three branches of this manifold and choose the ' $M$ theory circle' to be the fiber of the Hopf bundle in the 3-sphere

$$
\mathbf{S}^{1} \hookrightarrow \mathbf{S}^{3} \rightarrow \mathbf{S}^{2}
$$

we obtain the resolved conifold as a quotient space, $X / U(1) \cong \mathbf{S}^{2} \times \mathbb{R}^{4}$. More precisely, we obtain a resolved conifold with Ramond-Ramond 2-form flux and no D6-branes because the circle action has no fixed points in this case. This gives us one side of the brane/flux duality, namely the right-hand side on the diagram below:

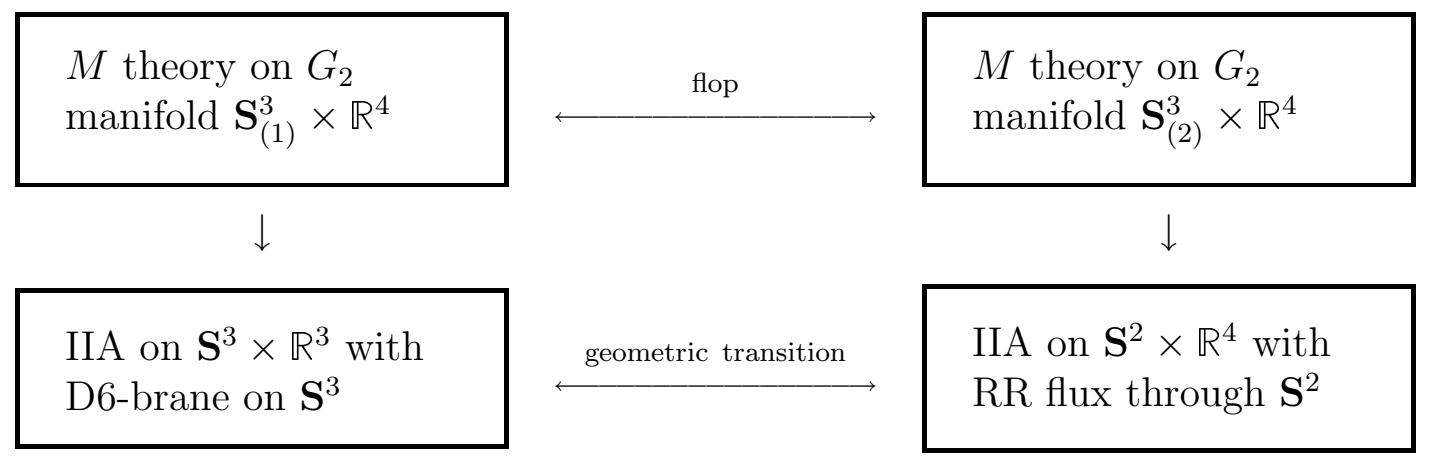

Now, let us follow the $G_{2}$ flop transition in $M$ theory on the manifold $X \cong \mathbf{S}^{3} \times \mathbb{R}^{3}$. As explained in the previous section, after the transition we obtain a $G_{2}$ manifold with similar topology, but the $M$ theory circle is now embedded in $\mathbb{R}^{4}$, rather than in $\mathbf{S}^{3}$. Acting on each $\mathbb{R}^{4}$ fiber, it yields $\mathbb{R}^{3}=\mathbb{R}^{4} / U(1)$ as a quotient space with a single fixed point at the origin of the $\mathbb{R}^{4}$ (see the discussion of $M$ theory on the Taub-NUT space in section 5.2). Applying this to each fiber of the $G_{2}$ manifold $X$, we obtain the deformed conifold as the quotient space, $X / U(1) \cong \mathbf{S}^{3} \times \mathbb{R}^{4}$, with the fixed point set $L=\mathbf{S}^{3}$. Since the latter is identified with the location of the space-filling D6-brane, we recover the other side of the brane/flux duality, illustrated in the above diagram.

Thus, we explained that the geometric transition — which is a highly non-trivial, non-perturbative phenomenon in string theory - can be understood as a $G_{2}$ flop transition in $M$ theory. Various aspects of this transition have been discussed in 

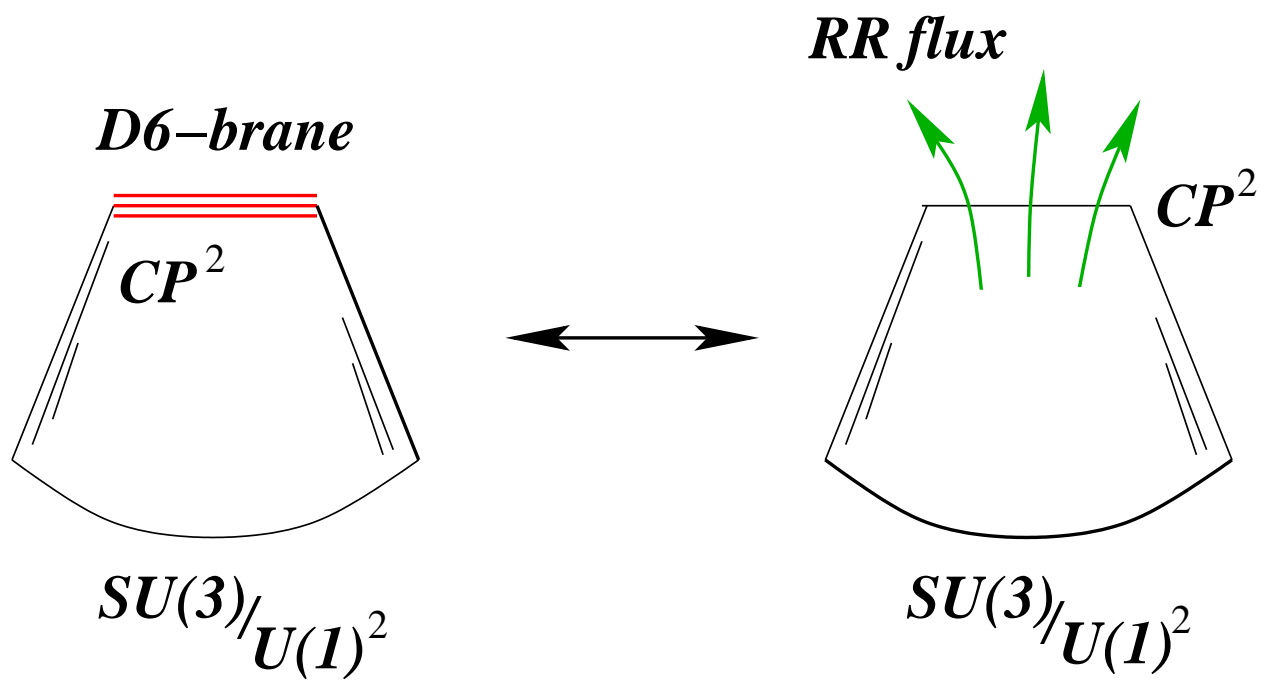

Figure 13: Geometric transition in IIA string theory connecting one branch of the $G_{2}$ manifold $\mathbb{C} \mathbf{P}^{2} \times \mathbb{R}^{3}$, where D6-branes are warpped around the $\mathbb{C P}^{2}$ cycle and another branch, where D6-branes are replaced by Ramond-Ramond 2-form flux through the $\mathbb{C} \mathbf{P}^{1} \subset \mathbb{C} \mathbf{P}^{2}$.

$[6,7,8,31,32,33,34,92]-[103]$. As we show next, there is a similar relation between the phase transition in $G_{2}$ holonomy manifold $X=\mathbb{C P}^{2} \times \mathbb{R}^{3}$ and the $\operatorname{Spin}(7)$ conifold transition, discussed in the previous section.

- $\mathrm{G}_{2} \rightarrow \operatorname{Spin}(\mathbf{7})$ : Consider type IIA string theory on the $G_{2}$ holonomy manifold

$$
\mathbb{C} \mathbf{P}^{2} \times \mathbb{R}^{3}
$$

which is obtained by resolving the cone over $S U(3) / U(1)^{2}$. As was discussed in the previous section, the corresponding moduli space has three classical branches connected by a singular phase transition. Motivated by the geometric transition in the conifold example, one could wrap an extra D6-brane over the $\mathbb{C P}^{2}$ cycle and ask a similar question: "What happens if one tries to go through a phase transition?".

Using arguments similar to [7], one finds that the transition is again possible, via $M$ theory on a $\operatorname{Spin}(7)$ manifold [26]. More precisely, after the geometric transition one finds type IIA string theory in a different phase of the $G_{2}$ manifold (7.128), where the D6-brane is replaced by $\mathrm{RR}$ flux through $\mathbb{C} \mathbf{P}^{1} \subset \mathbb{C} \mathbf{P}^{2}$. This leads to a fibration:

$$
\mathrm{S}^{1} \hookrightarrow \mathrm{S}^{5} \rightarrow \mathbb{C} \mathbf{P}^{2}
$$

Hence the $M$ theory lift of this configuration gives a familiar $\operatorname{Spin}(7)$ conifold,

$$
X \cong \mathbf{S}^{5} \times \mathbb{R}^{3} .
$$


Similarly, one can identify the lift of $\mathbb{C} \mathbf{P}^{2} \times \mathbb{R}^{3}$ with a D6-brane wrapped around $\mathbb{C} \mathbf{P}^{2}$ as the $\operatorname{Spin}(7)$ manifold $\mathbb{C} \mathbf{P}^{2} \times \mathbb{R}^{4}$, which is another topological phase of the $\operatorname{Spin}(7)$ conifold. Summarizing, we find that the conifold transition in $M$ theory on a Spin(7) manifold is related to a geometric transition in IIA string theory on the $G_{2}$ manifold (7.128) with branes/fluxes, as shown in the diagram below:
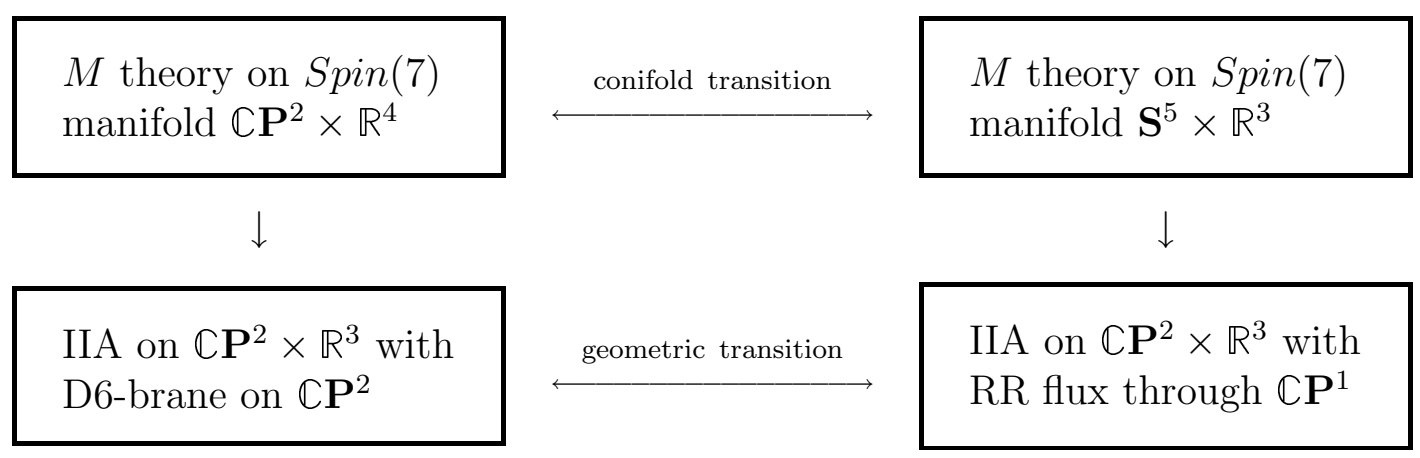

However, unlike its prototype with larger supersymmetry, this transition does not proceed smoothly. 


\section{Quantum Super-Yang-Mills from $G_{2}$ Manifolds}

Combining the results of the previous sections, now we move on to study the relation between quantum super Yang-Mills theory in four dimensions and properties of $M$ theory on $G_{2}$-manifolds. The results of this section are based upon $[6,7,8,104]$. We will be studying the physics of $M$ theory on the $G_{2}$-manifolds with ADE-singularities whose construction we described in sections 3.2 and 5.1. Specifically, we shall consider the $G_{2}$-manifolds that are obtained as quotients of $\mathbb{R}^{4} \times S^{3}$ by $\mathbb{T}_{\mathrm{ADE}}$. We begin by reviewing the basic properties of super Yang-Mills theory. We then go on to describe how these features are reflected in $M$ theory. We first show how membrane instantons can be seen to generate the superpotential of the theory. Then we go on to exhibit confinement and a mass gap semi-classically in $M$ theory.

\subsection{Super Yang-Mills Theory.}

For completeness and in order to compare easily with $M$ theory results obtained later we briefly give a review of $\mathcal{N}=1$ pure super Yang-Mills theory. We begin with gauge group $S U(n) . \mathcal{N}=1 S U(n)$ super Yang-Mills theory in four dimensions is an extensively studied quantum field theory. The classical Lagrangian for the theory is

$$
\mathcal{L}=-\frac{1}{4 g^{2}}\left(F_{\mu \nu}^{a}\right)^{2}+\frac{1}{g^{2}} \bar{\lambda}^{a} i \mathcal{D} \lambda^{a}+i \frac{\theta}{32 \pi^{2}} F_{\mu \nu}^{a} \tilde{F}^{a \mu \nu}
$$

$F$ is the gauge field strength and $\lambda$ is the gaugino field.

It is widely believed that this theory exhibits dynamics very similar to those of ordinary QCD: confinement, chiral symmetry breaking, a mass gap. There are $n$ supersymmetric vacua. Supersymmetry constrains the dynamics of the theory so strongly, that the values of the low energy effective superpotential in the $n$ vacua is known. These are of the form

$$
W_{e f f} \sim \mu^{3} e^{2 \pi i \tau / n}
$$

here $\tau$ is the complex coupling constant,

$$
\tau=\frac{\theta}{2 \pi}+i \frac{4 \pi}{g^{2}}
$$

and $\mu$ the cut-off scale. Shifting $\theta$ by $2 \pi$ gives $n$ different values for $W$.

In particular, the form of this potential suggests that it is generated by dynamics associated with "fractional instantons", i.e. instantonic objects in the theory whose quantum numbers are formally of instanton number $\frac{1}{n}$. Such states are also closely related to the spontaneously broken chiral symmetry of the theory. Let us briefly also review some of these issues here. 
Under the $U(1)$ R-symmetry of the theory, the gauginos transform as

$$
\lambda \rightarrow e^{i \alpha} \lambda
$$

This is a symmetry of the classical action but not of the quantum theory (as can easily be seen by considering the transformation of the fermion determinant in the path integral). However, if the above transformation is combined with a shift in the theta angle of the form

$$
\tau \rightarrow \tau+\frac{2 n}{2 \pi} \alpha
$$

then this cancels the change in the path integral measure. This shift symmetry is a bona fide symmetry of the physics if $\alpha=\frac{2 \pi}{2 n}$, so that even in the quantum theory a $\mathbf{Z}_{\mathbf{2 n}}$ symmetry remains. Associated with this symmetry is the presence of a non-zero value for the following correlation function,

$$
\left\langle\lambda \lambda\left(x_{1}\right) \lambda \lambda\left(x_{2}\right) \ldots \lambda \lambda\left(x_{n}\right)\right\rangle
$$

which is clearly invariant under the $\mathbf{Z}_{\mathbf{2 n}}$ symmetry. This correlation function is generated in the 1-instanton sector and the fact that $2 n$ gauginos enter is due to the fact that an instanton of charge 1 generates $2 n$ chiral fermion zero modes.

Cluster decomposition implies that the above correlation function decomposes into ' $n$ constituents' and therefore there exists a non-zero value for the gaugino condensate:

$$
\langle\lambda \lambda\rangle \neq 0
$$

Such a non-zero expectation value is only invariant under a $\mathbf{Z}_{\mathbf{2}}$ subgroup of $\mathbf{Z}_{\mathbf{2 n}}$ implying that the discrete chiral symmetry has been spontaneously broken. Consequently this implies the existence of $n$ vacua in the theory.

In fact, it can be shown that

$$
\langle\lambda \lambda\rangle=16 \pi i \frac{\partial}{\partial \tau} W_{e f f} \sim-\frac{32 \pi^{2}}{n} c \mu^{3} e^{2 \pi i \tau / n}
$$

In view of the above facts it is certainly tempting to propose that 'fractional instantons' generate the non-zero gaugino condensate directly. But this is difficult to see directly in super Yang-Mills on $\mathbb{R}^{\mathfrak{B}, 1}$. We will return to this point later.

More generally, if we replace the $S U(n)$ gauge group by some other gauge group $H$, then the above statements are also correct but with $n$ replaced everywhere with $c_{2}(H)$ the dual Coxeter number of $H$. For A-D-E gauge groups $c_{2}(H)=\sum_{i=1}^{r+1} a_{i}$, where $r$ is the rank of the gauge group and the $a_{i}$ are the Dynkin indices of the affine Dynkin diagram associated to $H$. For $A_{n}$, all the $a_{i}=1$; for $D_{n}$ groups the four 'outer' nodes have index 1 whilst the rest have $a_{i}=2$. $E_{6}$ has indices $(1,1,1,2,2,2,3)$, $E_{7}$ has $(1,1,2,2,2,3,3,4)$ whilst $E_{8}$ has indices $(1,2,2,3,3,4,4,5,6)$. 


\subsection{Theta angle and Coupling Constant in M theory.}

The physics of $M$ theory supported near the singularities of $\mathbb{C}^{\mathbb{2}} / \mathbb{T} \times \mathbb{R}^{6, \mathbb{1}}$ is described by super Yang-Mills theory on $\mathbb{R}^{6, \mathbb{1}}$. The gauge coupling constant of the theory is given by

$$
\frac{1}{g_{7 d}^{2}} \sim \frac{1}{l_{p}^{3}}
$$

where $l_{p}$ is the eleven dimensional Planck length. In seven dimensions, one analog of the theta angle in four dimensions is actually a three-form $\Theta$. The reason for this is the seven dimensional interaction

$$
L_{I} \sim \Theta \wedge F \wedge F
$$

(with $F$ the Yang-Mills field strength). In $M$ theory $\Theta$ is given by $C$, the three-form potential for the theory.

If we now take $M$ theory on our $G_{2}$-manifold $\mathbb{R}^{4} / \llbracket \times W$ we have essentially compactified the seven dimensional theory on $W$ and the four dimensional gauge coupling constant is roughly given by

$$
\frac{1}{g_{4 d}^{2}} \sim \frac{V_{W}}{l_{p}^{3}}
$$

where $V_{W}$ is the volume of $W$. The four dimensional theta angle can be identified as

$$
\theta=\int_{W} C
$$

The above equation is correct because under a global gauge transformation of $C$ which shifts the above period by $2 \pi$ times an integer - a transformation which is a symmetry of $M$ theory - $\theta$ changes by $2 \pi$ times an integer. Such shifts in the theta angle are also global symmetries of the field theory.

Thus the complex gauge coupling constant of the effective four dimensional theory may be identified as the $\tau$ parameter of $M$ theory

$$
\tau=\int_{W} \frac{C}{2 \pi}+i \frac{\Phi}{l_{p}^{3}}
$$

This is of course entirely natural, since $\tau$ is the only parameter in $M$ theory on this space!

\subsection{Superpotential in $M$ theory.}

There is a very elegant way to calculate the superpotential of super Yang-Mills theory on $\mathbb{R}^{\mathbb{B}, \mathbb{1}}$ by first compactifying it on a circle to three dimensions [106]. The three 
dimensional theory has a perturbative expansion since the Wilson lines on the circle behave as Higgs fields whose vev's break the gauge symmetry to the maximal torus. The theory has a perturbative expansion in the Higss vevs, which can be used to compute the superpotential of the compactified theory. One then takes the four dimensional limit. In order to compute the field theory superpotential we will mimick this idea in $M$ theory [5]. Compactifying the theory on a small circle is equivalent to studying pertubative Type IIA string theory on our $G_{2}$-manifold.

\section{Type IIA theory on $X=\mathbb{R}^{4} / \mathbb{\varpi}_{\mathbb{A D E}} \times S^{3}$}

Consisder Type IIA string theory compactified to three dimensions on a seven manifold $X$ with holonomy $G_{2}$. If $X$ is smooth we can determine the massless spectrum of the effective supergravity theory in three dimensions as follows. Compactification on $X$ preserves four of the 32 supersymmetries in ten dimensions, so the supergravity theory has three dimensional $\mathcal{N}=2$ local supersymmetry. The relevant bosonic fields of the ten dimensional supergravity theory are the metric, $B$-field, dilaton plus the RamondRamond one- and three-forms. These we will denote by $g, B, \phi, A_{1}, A_{3}$ respectively. Upon Kaluza-Klein reduction the metric gives rise to a three-metric and $b_{3}(X)$ massless scalars. The latter parametrise the moduli space of $G_{2}$-holonomy metrics on $X$. $B$ gives rise to $b_{2}(X)$ periodic scalars $\varphi_{i}$. $\phi$ gives a three dimensional dilaton. $A_{1}$ reduces to a massless vector, while $A_{3}$ gives $b_{2}(X)$ vectors and $b_{3}(X)$ massless scalars. In three dimensions a vector is dual to a periodic scalar, so at a point in moduli space where the vectors are free we can dualise them. The dual of the vector field originating from $A_{1}$ is the period of the RR 7 -form on $X$, whereas the duals of the vector fields coming from $A_{3}$ are given by the periods of the RR 5 -form $A_{5}$ over a basis of 5-cycles which span the fifth homology group of $X$. Denote these by scalars by $\sigma_{i}$. All in all, in the dualised theory we have in addition to the supergravity multiplet, $b_{2}(X)+b_{3}(X)$ scalar multiplets. Notice that $b_{2}(X)$ of the scalar multiplets contain two real scalar fields, both of which are periodic.

Now we come to studying the Type IIA theory on $X=\mathbb{R}^{4} / \mathbb{I}_{\mathbb{A D E}} \times S^{3}$. Recall that $X=\mathbb{R}^{4} / \mathbb{I}_{\mathbb{A D E}} \times S^{3}$ is defined as an orbifold of the standard spin bundle of $S^{3}$. To determine the massless spectrum of IIA string theory on $X$ we can use standard orbifold techniques. However, the answer can be phrased in a simple way. $X$ is topologically $\mathbb{R}^{4} / \mathbb{\square}_{A \mathbb{A E E}} \times S^{3}$. This manifold can be desingularised to give a smooth seven manifold $M^{\varpi_{A D E}}$ which is topologically $X^{\varpi_{A D E}} \times S^{3}$, where $X^{\varpi_{A D E}}$ is homeomorphic to an $A L E$ space. The string theoretic cohomology groups of $X$ are isomorphic to the usual cohomology groups of $M^{\varpi_{A D E}}$. The reason for this is simple: $X$ is a global orbifold of $S\left(S^{3}\right)$. The string theoretic cohomology groups count massless string states in the 
orbifold CFT. The massless string states in the twisted sectors are localised near the fixed points of the action of $\mathbb{T}_{\mathbb{A D E}}$ on the spin bundle. Near the fixed points we can work on the tangent space of $S\left(S^{3}\right)$ near a fixed point and the action of $\mathbb{\square}_{\text {ADE }}$ there is just its natural action on $\mathbb{R}^{4} \times \mathbb{R}^{3}$.

Note that blowing up $X$ to give $M^{\mathbb{A A D E}_{\text {A }}}$ cannot give a metric with $G_{2}$-holonomy which is continuosly connected to the singular $G_{2}$-holonomy metric on $X$, since this would require that the addition to homology in passing from $X$ to $M^{\varpi_{\mathbb{A D E}}}$ receives contributions from four-cycles. This is necessary since these are dual to elements of $H^{3}(M)$ which generate metric deformations preserving the $G_{2}$-structure. This argument does not rule out the possibility that $M^{\mathbb{A}_{\mathrm{ADE}}}$ admits 'disconnected' $G_{2}$-holonomy metrics, but is consistent with the fact that pure super Yang-Mills theory in four dimensions does not have a Coulomb branch.

The important points to note are that the twisted sectors contain massless states consisting of $r$ scalars and $r$ vectors where $r$ is the rank of the corresponding ADE group associated to $\mathbb{~}$. The $r$ scalars can intuitively thought of as the periods of the $B$ field through $r$ two cycles. In fact, for a generic point in the moduli space of the orbifold conformal field theory the spectrum contains massive particles charged under the $r$ twisted vectors. These can be interpreted as wrapped D2-branes whose quantum numbers are precisely those of $W$-bosons associated with the breaking of an ADE gauge group to $U(1)^{r}$. This confirms our interpretation of the origin of this model from $M$ theory: the values of the $r B$-field scalars can be interpreted as the expectation values of Wilson lines around the eleventh dimension associated with this symmetry breaking. At weak string coupling and large $S^{3}$ volume these states are very massive and the extreme low energy effective dynamics of the twisted sector states is described by $\mathcal{N}=2 U(1)^{r}$ super Yang-Mills in three dimensions. Clearly however, the underlying conformal field theory is not valid when the $W$-bosons become massless. The appropriate description is then the pure super Yang-Mills theory on $\mathbb{R}^{2, \mathbb{1}} \times S^{1}$ which corresponds to a sector of $M$ theory on $X \times S^{1}$. In this section however, our strategy will be to work at a generic point in the CFT moduli space which corresponds to being far out along the Coulomb branch of the gauge theory. We will attempt to calculate the superpotential there and then continue the result to four dimensions. This exactly mimics the strategy of [106] in field theory. Note that we are implicitly ignoring gravity here. More precisely, we are assuming that in the absence of gravitational interactions with the twisted sector, the low energy physics of the twisted sectors of the CFT is described by the Coulomb branch of the gauge theory. This is natural since the twisted sector states are localised at the singularities of $J \times \mathbb{R}^{2, \mathbb{1}}$ whereas the gravity propagates in bulk.

In this approximation, we can dualise the photons to obtain a theory of $r$ chiral 
multiplets, each of whose bosonic components $(\boldsymbol{\varphi}$ and $\boldsymbol{\sigma})$ is periodic. But remembering that this theory arose from a non-Abelian one we learn that the moduli space of classical vacua is

$$
\mathcal{M}_{c l}=\frac{\mathbb{C}^{r}}{\Lambda_{W}^{\mathbb{C}} \rtimes W_{g}}
$$

where $\Lambda_{W}^{\mathbb{C}}$ is the complexified weight lattice of the ADE group and $W_{g}$ is the Weyl group.

We can now ask about quantum effects. In particular is there a non-trivial superpotential for these chiral multiplets? In a theory with four supercharges BPS instantons with only two chiral fermion zero modes can generate a superpotential. Are there instantons in Type IIA theory on J ? BPS instantons come from branes wrapping supersymmetric cycles and Type IIA theory on a $G_{2}$-holonomy space can have instantons corresponding to D6-branes wrapping the space itself or D2-brane instantons which wrap supersymmetric 3 -cycles. For smooth $G_{2}$-holonomy manifolds these were studied in [107]. In the case at hand the D6-branes would generate a superpotential for the dual of the graviphoton multiplet which lives in the gravity multiplet but since we wish to ignore gravitational physics for the moment, we will ignore these. In any case, since $X$ is non-compact, these configurations have infinite action. The D2-branes on the other hand are much more interesting. They can wrap the supersymmetric $S^{3}$ over which the singularities of $X$ are fibered. We can describe the dynamics of a wrapped D2-brane as follows. At large volume, where the sphere becomes flatter and flatter the world-volume action is just the so called 'quiver gauge theory' described in [64]. Here we should describe this theory not just on $S^{3}$ but on a supersymmetric $S^{3}$ embedded in a space with a non-trivial $G_{2}$-holonomy structure. The upshot is that the world-volume theory is in fact a cohomological field theory [108] so we can trust it for any volume as long as the ambient space has $G_{2}$-holonomy. This is because the supersymmetries on the world-volume are actually scalars on $S^{3}$ and so must square to zero.

Note that, since we are ignoring gravity, we are implicitly ignoring higher derivative corrections which could potentially also affect this claim. Another crucial point is that the $S^{3}$ which sits at the origin in $\mathbb{R}^{4}$ in the covering space of $X$ is the supersymmetric cycle, and the spheres away from the origin are not supersymmetric, so that the BPS wrapped D2-brane is constrained to live on the singularities of $X$. In the quiver gauge theory, the origin is precisely the locus in moduli space at which the single D2-brane can fractionate (according to the quiver diagram) and this occurs by giving expectation values to the scalar fields which parametrise the Coulomb branch which corresponds to the position of our D2-brane in the dimensions normal to $X$.

What contribution to the superpotential do the fractional D2-branes make? To 
answer this we need to identify the configurations which possess only two fermionic zero modes. We will not give a precise string theory argument for this, but using the correspondence between this string theory and field theory will identify exactly which D-brane instantons we think are responsible for generating the superpotential. This may sound like a strong assumption, but as we hope will become clear, the fact that the fractional D2-branes are wrapped D4-branes is actually anticipated by the field theory! This makes this assumption, in our opinion, somewhat weaker and adds credence to the overall picture being presented here.

In [109], it was shown that the fractionally charged D2-branes are actually D4-branes which wrap the 'vanishing' 2-cycles at the origin in $\mathbb{R}^{4} / \llbracket$. More precisely, each individual fractional D2-brane, which originates from a single D2-brane possesses D4-brane charge, but the total configuration, since it began life as a single D2-brane has zero D4-brane charge. The possible contributions to the superpotential are constrained by supersymmetry and must be given by a holomorphic function of the $r$ chiral superfields and also of the holomorphic gauge coupling constant $\tau$ which corresponds to the complexified volume of the $S^{3}$ in eleven dimensional $M$ theory. We have identified above the bosonic components of the chiral superfields above. $\tau$ is given by

$$
\tau=\int \Phi+i C
$$

where $\Phi$ is the $G_{2}$-structure defining 3 -form on $X$. The period of the $M$ theory 3 -form through $S^{3}$ plays the role of the theta angle.

The world-volume action of a D4-brane contains the couplings

$$
L=B \wedge A_{3}+A_{5}
$$

Holomorphy dictates that there is also a term

$$
B \wedge \Phi
$$

so that the combined terms are written as

$$
B \wedge \tau+A_{5}
$$

Since the fourbranes wrap the 'vanishing cycles' and the $S^{3}$ we see that the contribution of the D4-brane corresponding to the $k$-th fractional D2 takes the form

$$
S=-\boldsymbol{\beta}_{\boldsymbol{k}} \cdot \boldsymbol{z}
$$

where we have defined

$$
z=\tau \varphi+\sigma
$$


and the $\boldsymbol{\beta}_{\boldsymbol{k}}$ are charge vectors. The $r$ complex fields $\boldsymbol{z}$ are the natural holomorphic functions upon which the superpotential will depend.

The wrapped D4-branes are the magnetic duals of the massive D2-branes which we identified above as massive $W$-bosons. As such they are magnetic monopoles for the original ADE gauge symmetry. Their charges are therefore given by an element of the co-root lattice of the Lie algebra and thus each of the $r+1 \boldsymbol{\beta}$ 's is a rank $r$ vector in this space. Choosing a basis for this space corresponds to choosing a basis for the massless states in the twisted sector Hilbert space which intuitively we can think of as a basis for the cohomology groups Poincare dual to the 'vanishing' 2-cycles. A natural basis is provided by the simple co-roots of the Lie algebra of ADE, which we denote by $\boldsymbol{\alpha}_{\boldsymbol{k}}^{*}$ for $k=1, \ldots, r$. This choice is natural, since these, from the field theory point of view are the fundamental monopole charges.

At this point it is useful to mention that the $r$ wrapped D4-branes whose magnetic charges are given by the simple co-roots of the Lie algebra correspond in field theory to monopoles with charges $\boldsymbol{\alpha}_{\boldsymbol{k}}^{*}$ and each of these is known to possess precisely the right number of zero modes to contribute to the superpotential. Since we have argued that in a limit of the Type IIA theory on $X$, the dynamics at low energies is governed by the field theory studied in [106] it is natural to expect that these wrapped fourbranes also contribute to the superpotential. Another striking feature of the field theory is that these monopoles also possess a fractional instanton number - the second Chern number of the gauge field on $\mathbb{R}^{2, \mathbb{1}} \times S^{1}$. These are precisely in correspondence with the fractional D2-brane charges. Thus, in this sense, the field theory anticipates that fractional branes are wrapped branes.

In the field theory on $\mathbb{R}^{2, \mathbb{1}} \times S^{1}$ it is also important to realise that there is precisely one additional BPS state which contributes to the superpotential. The key point is that this state, unlike the previously discussed monopoles have dependence on the periodic direction in spacetime. This state is associated with the affine node of the Dynkin diagram. Its monopole charge is given by

$$
-\Sigma_{k=1}^{r} \boldsymbol{\alpha}_{\boldsymbol{k}}^{*}
$$

and it also carries one unit of instanton number.

The action for this state is

$$
S=\Sigma_{k=1}^{r} \boldsymbol{\alpha}_{\boldsymbol{k}}^{*} \boldsymbol{z}-2 \pi i \tau
$$

Together, these $r+1$ BPS states can be regarded as fundamental in the sense that all the other finite action BPS configurations can be thought of as bound states of them. 
Thus, in the correspondence with string theory it is also natural in the same sense as alluded to above that a state with these corresponding quantum numbers also contributes to the superpotential. It may be regarded as a bound state of anti-D4-branes with a charge one D2-brane. In the case of $S U(n)$ this is extremely natural, since the total D4/D2-brane charge of the $r+1$ states is zero/one, and this is precisely the charge of the D2-brane configuration on $S^{3}$ whose world-volume action is the quiver gauge theory for the affine Dynkin diagram for $S U(n)$. In other words, the entire superpotential is generated by a single D2-brane which has fractionated.

In summary, we have seen that the correspondence between the Type IIA string theory on $X$ and the super Yang-Mills theory on $\mathbb{R}^{2, \mathbb{1}} \times S^{1}$ is quite striking. Within the context of this correspondence we considered a smooth point in the moduli space of the perturbative Type IIA CFT, where the spectrum matches that of the Yang-Mills theory along its Coulomb branch. On the string theory side we concluded that the possible instanton contributions to the superpotential are from wrapped D2-branes. Their world volume theory is essentially topological, from which we concluded that they can fractionate. As is well known, the fractional D2-branes are really wrapped fourbranes. In the correspondence with field theory, the wrapped fourbranes are magnetic monopoles, whereas the D2-branes are instantons. Thus if, these branes generate a superpotential they correspond, in field theory to monopole-instantons. This is exactly how the field theory potential is known to be generated. We thus expect that the same occurs in the string theory on $X$.

Finally, the superpotential generated by these instantons is of affine-Toda type and is known to possess $c_{2}(\mathrm{ADE})$ minima corresponding to the vacua of the ADE super Yang-Mills theory on $\mathbb{R}^{\mathbb{B}, \mathbb{1}}$. The value of the superpotential in each of these vacua is of the form $e^{\frac{2 \pi i \tau}{c_{2}}}$. As such it formally looks as though it was generated by fractional instantons, and in this context fractional $M 2$-brane instantons. This result holds in the four dimensional $M$ theory limit because of holomorphy.

Let us demonstrate the vacuum structure in the simple case when the gauge group is $S U(2)$. Then there is only one scalar field, $z$. There are two fractional D2-brane instantons whose actions are

$$
S_{1}=-z \quad \text { and } \quad S_{2}=z-2 \pi i \tau
$$

Both of these contribute to the superpotential as

$$
W=e^{-S_{1}}+e^{-S_{2}}
$$

Defining $z=\ln Y$ we have

$$
W=Y+\frac{e^{2 \pi i \tau}}{Y}
$$


The critical points of $W$ are

$$
Y= \pm e^{\frac{2 \pi i \tau}{2}}
$$

This result about the superpotential suggests strongly that there is a limit of $M$ theory near an ADE singularity in a $G_{2}$-manifold which is precisely super Yang-Mills theory. We will now go on to explore other limits of this $M$ theory background.

\subsection{M theory Physics on ADE-singular $G_{2}$-manifolds.}

We saw previously in section 7 , that before taking the quotient by $\mathbb{\pi}$, the $M$ theory physics on $\mathbb{R}^{4} \times S^{3}$, with its $G_{2}$-metric, was smoothly varying as a function of $\tau$. In fact the same is true in the case with ADE-singularities. One hint for this was that we explicitly saw just now that the superpotential is non-zero in the various vacua and this implies that the $C$-field is non-zero. This suggestion was concretely proven in [8], see also [110].

Before orbifolding by $\llbracket$ we saw there were three semiclassical limits of $M$ theory in the space parameterised by $\tau$. These were described by $M$ theory on three large and smooth $G_{2}$-manifolds $X_{i}$, all three of which were of the form $\mathbb{R}^{4} \times S^{3}$. There are also three semiclassical i.e. large volume $G_{2}$-manifolds when we orbifold by $\mathbb{~}$. These are simply the quotients by $\mathbb{0}$ of the $X_{i}$. One of these is the $G_{2}$-manifold $\mathbb{R}^{4} / \mathbb{\square}_{\mathbb{A D E}} \times S^{3}$. The other two are both of the form $S^{3} / \mathbb{\mathbb { A D E }}_{\mathbb{A} \mathbb{E}} \times \mathbb{R}^{4}$. To see this, note that the three $S^{3}$ 's in the three $G_{2}$-manifolds $X_{i}$ of the form $\mathbb{R}^{4} \times S^{3}$ correspond to the three $S^{3}$ factors in $G=S U(2)^{3}=S^{3} \times S^{3} \times S^{3}$, c.f. section 3.2. $\mathbb{T}_{\mathbb{A D E}}$ is a subgroup of one of these $S^{3}$ 's. If $\mathbb{T}_{\mathbb{A D E}}$ acts on the $\mathbb{R}^{4}$ factor of $X_{1}$ in the standard way, then it must act on $S^{3}$ in $X_{2}$ - since $X_{2}$ can be thought of as the same manifold but with the two $S^{3}$ 's at infinity exchanged. Then, because of the permutation symmetry it also acts on the $S^{3}$ in $X_{3}$. Thus, $X_{2} / \mathbb{\varpi}_{\mathbb{A D E}}$ is isomorphic to $S^{3} / \mathbb{\llbracket}_{\mathbb{A D E}} \times \mathbb{R}^{4}$, as is $X_{3}$. Thus, varying $\tau$ can take us from $M$ theory on the singular $G_{2}$-manifold $X_{1} / \mathbb{I}_{\mathbb{A D E}}$ to the smooth $G_{2}$-manifolds $X_{2,3} / \mathbb{\varpi}_{\text {ADE }}$.

On $X_{1} / \mathbb{}_{\text {ADEE }}$ in the large volume limit, we have a semi-classical description of the four dimensional physics in terms of perturbative super Yang-Mills theory. But, at extremely low energies, this theory becomes strongly coupled, and is believed to confine and get a mass gap. So, apart from calculating the superpotential in each vacuum, we can't actually calculate the spectrum here.

What about the physics in the other two semiclassical limits, namely large $X_{2,3} / \mathbb{\complement}_{\mathbb{A D E}}$ ? These $G_{2}$-manifolds are completely smooth. So supergravity is a good approximation to the $M$ theory physics. What do we learn about the $M$ theory physics in this approximation? 


\subsection{Confinement from $G_{2}$-manifolds.}

If it is true that the qualitative physics of $M$ theory on $X_{2} / \mathbb{\varpi}_{\mathbb{A D E}}$ and $X_{3} / \widetilde{\mathbb{A D E}}_{\mathbb{A} \text { is }}$ the same as that of $M$ theory on $X_{1} / \mathbb{T}_{\mathbb{A D E}}$, then some of the properties of super Yang-Mills theory at low energies ought to be visible. The gauge theory is believed to confine ADE-charge at low energies. If a gauge theory confines in four dimensions, electrically charged confining flux tubes (confining strings) should be present. If the classical fields of the gauge theory contain only fields in the adjoint representation of the gauge group $G$, then these strings are charged with respect to the center of $G, Z(G)$. Can we see these strings in $M$ theory on $X_{2} / \mathbb{\square}_{\mathbb{A D E}}$ ? As shown in [104], the answer is yes.

The natural candidates for such strings are $M 2$-branes which wrap around 1-cycles in $X_{2} / \mathbb{\varpi}_{\mathbb{A D E}}$ or $M 5$-branes which wrap 4-cycles in $X_{2} / \mathbb{\varpi}_{\mathbb{A D E}}$. Since $X_{2} / \mathbb{\varpi}_{\mathbb{A D E}}$ is homeomorphic to $S^{3} / \mathbb{\square}_{\mathbb{A D E}} \times \mathbb{R}^{4}$ which is contractible to $S^{3} / \mathbb{\square}_{\mathbb{A D E}}$, the homology groups of $X_{2} / \mathbb{}_{\mathbb{A D E}}$ are the same as those of the three-manifold $S^{3} / \mathbb{\varpi}_{\mathbb{A D E}}$. Thus, our space has no four cycles to speak of, so the confining strings can only come from $M 2$-branes wrapping one-cycles in $S^{3} / \mathbb{\varpi}_{\mathbb{A D E}}$. The string charges are classified by the first homology group $H_{1}\left(S^{3} / \widetilde{\Gamma}_{\text {ADEE }}, \mathbb{Z}\right)$. For any manifold, the first homology group is isomorphic to the abelianisation of its fundamental group, $\Pi_{1}$. The abelianisation is obtained by setting all commutators in $\Pi_{1}$ to be trivial i.e.

$$
H_{1}(M, \mathbb{Z}) \cong \frac{\pi_{1}(M)}{\left[\pi_{1}(M), \pi_{1}(M)\right]}
$$

The fundamental group of $S^{3} / \mathbb{}_{\mathbb{A D E}}$ is $\mathbb{\mathbb { A D E E }}_{\mathbb{A D E}}$. Hence, in order to calculate the charges of our candidate confining strings we to compute the abelianisations of all of the finite subgroups of $S U(2)$.

$\mathbb{}_{\mathbb{A}_{\mathfrak{m}-1}} \cong \mathbb{Z}_{\mathbb{m}}$. The gauge group is locally $S U(n)$. Since $\mathbb{Z}_{\mathbb{m}}$ is abelian, its commutator subgroup is trivial and hence the charges of our strings take values in $\mathbb{Z}_{\mathrm{m}}$. Since this is isomorphic to the center of $S U(n)$ this is the expected answer for a confining $S U(n)$ theory.

For $\mathbb{T} \cong \mathbb{D}_{\mathbb{k}-2}$, the binary dihedral group of order $4 k-8$, the local gauge group of the Yang-Mills theory is $S O(2 k)$. We remind that the binary dihedral group is generated by two elements $\alpha$ and $\beta$ which obey the relations

$$
\begin{gathered}
\alpha^{2}=\beta^{k-2} \\
\alpha \beta=\beta^{-1} \alpha \\
\alpha^{4}=\beta^{2 k-4}=1
\end{gathered}
$$


To compute the abelianisation of $\mathbb{D}_{\mathbb{k}-\mathfrak{T}}$, we simply take these relations and impose that the commutators are trivial. From the second relation this implies that

$$
\beta=\beta^{-1}
$$

which in turn implies that

$$
\alpha^{2}=1 \quad \text { for } \quad k=2 p
$$

and

$$
\alpha^{2}=\beta \quad \text { for } \quad k=2 p+1
$$

Thus, for $k=2 p$ we learn that the abelianisation of $\mathbb{D}_{\mathbb{k}-2}$ is isomorphic to $\mathbb{Z}_{2} \times \mathbb{Z}_{2}$, whereas for $k=2 p+1$ it is isomorphic to $\mathbb{Z}_{4}$. These groups are respectively the centers of $\operatorname{Spin}(4 p)$ and $\operatorname{Spin}(4 p+2)$. This is the expected answer for the confining strings in $S O(2 k)$ super Yang-Mills which can be coupled to spinorial charges.

To compute the abelianisations of the binary tetrahedral (denoted $\mathbb{T}$ ), octahedral $(\mathbb{O})$ and icosahedral () groups which correspond respectively to $E_{6}, E_{7}$ and $E_{8}$ super Yang-Mills theory, we utilise the fact that the order of $F /[F, F]$ - with $F$ a finite group - is the number of inequivalent one dimensional representations of $G$. The representation theory of the finite subgroups of $S U(2)$ is described through the Mckay correspondence by the representation theory of the corresponding Lie algebras. In particular the dimensions of the irreducible representations of $\mathbb{\mathbb { T }}, \mathbb{O}$ and $\mathbb{\mathbb { a }}$ are given by the coroot integers (or dual Kac labels) of the affine Lie algebrae associated to $E_{6}, E_{7}$ or $E_{8}$ respectively. From this we learn that the respective orders of $\mathbb{T} /[\mathbb{T}, \mathbb{T}], \mathbb{O} /[\mathbb{O}, \mathbb{O}]$ and $\mathbb{\mathbb { D }} /[\mathbb{[}, \mathbb{\mathbb { Q }}]$ are three, two and one. Moreover, one can easily check that $\mathbb{T} /[\mathbb{T}, \mathbb{T}]$ and $\mathbb{O} /[\mathbb{O}, \mathbb{O}]$ are $\mathbb{Z}_{\mathfrak{B}}$ and $\mathbb{Z}_{\mathbb{Z}}$ respectively, by examining their group relations. Thus we learn that $\mathbb{T} /[\mathbb{\mathbb { T }}, \mathbb{\mathbb { T }}], \mathbb{O} /[\mathbb{O}, \mathbb{O}]$ and $\mathbb{\square}\left[[\mathbb{\square}, \mathbb{0}]\right.$ are, respectively isomorphic to the centers $Z\left(E_{6}\right), Z\left(E_{7}\right)$ and $Z\left(E_{8}\right)$ in perfect agreement with the expectation that the super Yang-Mills theory confines. Note that the $E_{8}$-theory does not confine, since the strings are uncharged.

This result is also natural from the following point of view. In the singular $X_{1} / \mathbb{\llbracket}_{\mathbb{A D E}}$ (where the actual gauge theory dynamics is) the gauge bosons correspond to M2-branes wrapped around zero-size cycles. When we vary $\tau$ away from the actual gauge theory limit until we reach $M$ theory on a large and smooth $X_{2} / \widetilde{I}_{\text {ADE }}$ the confining strings are also wrapped $M 2$-branes. In the gauge theory we expect the confining strings to be "built" from the excitations of the gauge fields themselves. In $M$ theory, the central role played by the gauge fields is actually played by the $M 2$-brane.

\subsection{Mass Gap from $G_{2}$-manifolds.}

We can also see the mass gap expected of the gauge theory, by studying the spectrum of $M$ theory on the smooth $G_{2}$-manifolds $X_{2} / \mathbb{\varpi}_{\mathbb{A D E}}$ and $X_{3} / \mathbb{T}_{\mathbb{A D E}}$. One can easliy show 
that there are no $L^{2}$-normalisable fluctuations of the $G_{2}$-holonomy metric on $X_{i}$. In order to see this, one has to look at the $L^{2}$ norm of the metric fluctuations,

$$
|\delta g|^{2}=\int_{X} d^{7} x \sqrt{g} g^{i i^{\prime}} g^{j j^{\prime}} \delta g_{i j} \delta g_{i^{\prime} j^{\prime}}
$$

From this expression, it follows that a deformation of an asymptotically conical metric like (3.26) is $L^{2}$ normalizable if and only if $\delta g / g$ goes to zero faster than $r^{-7 / 2}$, where $r$ is the radial coordinate. Using the explicit form (3.45) of the $G_{2}$ metric on $X_{i}$, we find $\delta g / g \sim r^{-3}$, where $\delta g$ is the variation of the metric (3.45) with respect to a change in $r_{0}$. Therefore, this deformation is not $L^{2}$-normalisable,

$$
|\delta g|^{2} \rightarrow \infty
$$

This implies that the spectrum of $M$ theory on the $X_{i}$ is massive. Since $X_{2,3} / \mathbb{T}_{\mathbb{A D E}}$ is a smooth quotient of $X_{2,3}$, the $M$ theory spectrum also has a mass gap. Since $M$ theory on $X_{2,3} / \mathbb{\complement}_{\mathbb{A D E}}$ is smoothly connected to $M$ theory on $X_{1} / \widetilde{\varpi}_{\mathbb{A D E}}$ by varying $\tau$, this demonstrates that pure super Yang-Mills theory has a mass gap.

\section{Acknowledgments}

Various topics covered in this review are based on the work done together with A. Brandhuber, J. Gomis, S. Gubser X. de la Ossa, D. Tong, J. Sparks, C. Vafa, E. Witten, S.-T. Yau, and E. Zaslow, whom we wish to thank for many useful discussions and collaboration. This report was largely completed during the period B.S.A. served as a research associate at New High Energy Theory Center, Rutgers University, and S.G. served as a Clay Mathematics Institute Long-Term Prize Fellow. We thank both institutions for their support. The work of S.G. is also supported in part by RFBR grant 04-02-16880 and RFBR grant for Young Scientists 02-01-06322. 


\section{References}

[1] J. Erler, P. Langacker, "Status of the Standard Model," hep-ph/9809352.

[2] S. Dimopoulos and H. Georgi, "Softly Broken Supersymmetry And SU(5)," Nucl. Phys. B 193, 150 (1981);

S. Dimopoulos, S. Raby and F. Wilczek, "Supersymmetry And The Scale Of Unification," Phys. Rev. D 24, 1681 (1981).

[3] P. Candelas, G. T. Horowitz, A. Strominger and E. Witten, "Vacuum Configurations For Superstrings," Nucl. Phys. B 258, 46 (1985).

[4] D. R. Morrison and C. Vafa, "Compactifications of F-Theory on Calabi-Yau Threefolds - I,II" Nucl. Phys. B 473, 74 (1996); Nucl. Phys. B 476, 437 (1996).

[5] B. S. Acharya, "M theory, Joyce orbifolds and super Yang-Mills," Adv. Theor. Math. Phys. 3, 227 (1999) [arXiv:hep-th/9812205].

[6] B. S. Acharya, "On realising $N=1$ super Yang-Mills in $M$ theory," arXiv:hepth/0011089.

[7] M. Atiyah, J. Maldacena and C. Vafa, "An M theory Flop as a Large $N$ Duality", J. Math. Phys. 42 (2001) 3209, hep-th/0011256.

[8] M. Atiyah and E. Witten, "M-Theory Dynamics On A Manifold Of $G_{2}$ Holonomy", hep-th/0107177.

[9] E. Witten, "Anomaly cancellation on $G_{2}$-manifolds," arXiv:hep-th/0108165.

[10] B. Acharya and E. Witten, "Chiral fermions from manifolds of $G_{2}$ holonomy," arXiv:hep-th/0109152.

[11] E. Witten, "Deconstruction, G(2) holonomy, and doublet-triplet splitting," hep$\mathrm{ph} / 0201018$.

[12] T. Friedmann and E. Witten, "Unification scale, proton decay, and manifolds of G(2) holonomy," hep-th/0211269.

[13] B. S. Acharya, "A moduli fixing mechanism in M theory," hep-th/0212294.

[14] B. S. Acharya, "Compactification with flux and Yukawa hierarchies," hep-th/0303234.

[15] M. Berger, "Sur les groupes d'holonomie des variétés á connexion affines et des variétés riemanniennes," Bulletin de la Société Mathématique de France, 83 (1955) 279.

[16] D.V. Alekseevsky, "Riemannian Spaces with Exceptional Holonomy," Funct. Anal. Appl. 2 (1968) 97.

R. Brown and A. Gray, "Riemannian Manifolds with Holonomy Group Spin(9)," Differential Geometry (in honor of Kentaro Yano), Tokyo (1972) 41.

[17] D. Joyce, "Compact Manifolds with Special Holonomy", Oxford University Press, 2000.

[18] R. Harvey and H.B. Lawson, Jr., "Calibrated geometries", Acta Math. 148 (1982) 47.

[19] R. Mclean, "Deformations of Calibrated Submanifolds", Comm. Anal. Geom. 6 (1998) 705-747. 
[20] S.-T. Yau, "On the Ricci curvature of a compact Kähler manifold and the MongeAmpére equations. I," Commun. Pure Appl. Math. 31 (1978) 339.

[21] R.L. Bryant, "Metrics with Exceptional Holonomy," Ann. Math. 126 (1987) 525.

[22] A. Kovalev, "Twisted connected sums and special Riemannian holonomy," math.dg/0012189.

[23] R. Bryant, S. Salamon, "On the Construction of some Complete Metrics with Exceptional Holonomy", Duke Math. J. 58 (1989) 829.

[24] G. W. Gibbons, D. N. Page, C. N. Pope, "Einstein Metrics on $S^{3}, \mathbb{R}^{3}$ and $\mathbb{R}^{4}$ Bundles," Commun.Math.Phys 127 (1990) 529-553.

[25] M. Cvetic, G.W. Gibbons, H. Lu, C.N. Pope, "Cohomogeneity One Manifolds of $\operatorname{Spin}(7)$ and G(2) Holonomy", hep-th/0108245.

[26] S. Gukov, J. Sparks and D. Tong, "Conifold transitions and five-brane condensation in $M$ theory on Spin(7) manifolds," hep-th/0207244.

[27] S. Gukov, S. T. Yau and E. Zaslow, "Duality and fibrations on G(2) manifolds," arXiv:hep-th/0203217.

[28] Z. W. Chong, M. Cvetic, G. W. Gibbons, H. Lu, C. N. Pope and P. Wagner, "General metrics of G(2) holonomy and contraction limits," arXiv:hep-th/0204064.

[29] N. Hitchin, "Stable forms and special metrics," math.DG/0107101.

[30] N. Hitchin, "The geometry of three-forms in six and seven dimensions," math.DG/0010054.

[31] A. Brandhuber, J. Gomis, S. S. Gubser and S. Gukov, "Gauge theory at large $N$ and new G(2) holonomy metrics", Nucl. Phys. B 611, 179 (2001), hep-th/0106034.

[32] M. Cvetic, G. W. Gibbons, H. Lu and C. N. Pope, " $M$ theory conifolds," Phys. Rev. Lett. 88, 121602 (2002) [arXiv:hep-th/0112098].

[33] A. Brandhuber, "G(2) holonomy spaces from invariant three-forms," Nucl. Phys. B 629, 393 (2002) [arXiv:hep-th/0112113].

[34] M. Cvetic, G. W. Gibbons, H. Lu and C. N. Pope, "A G(2) unification of the deformed and resolved conifolds," Phys. Lett. B 534, 172 (2002) [arXiv:hep-th/0112138].

[35] M. Cvetic, G.W. Gibbons, H. Lu, C.N. Pope, "New Complete Non-compact Spin(7) Manifolds", Nucl. Phys. B620 (2002) 29, hep-th/0103155.

[36] S. Gukov and J. Sparks, "M theory on Spin(7) manifolds. I', Nucl. Phys. B625 (2002) 3, hep-th/0109025.

[37] H. Kanno, Y. Yasui, "On Spin(7) holonomy metric based on $S U(3) / U(1) I$ and IF", hep-th/0108226 and hep-th/0111198.

[38] J. M. Maldacena and C. Nunez, "Supergravity description of field theories on curved manifolds and a no go theorem," Int. J. Mod. Phys. A 16 (2001) 822, hep-th/0007018. J. M. Maldacena and C. Nunez, "Towards the large $N$ limit of pure $N=1$ super Yang Mills," Phys. Rev. Lett. 86 (2001) 588, hep-th/0008001. 
[39] J. D. Edelstein and C. Nunez, "D6 branes and M-theory geometrical transitions from gauged supergravity," JHEP 0104 (2001) 028, hep-th/0103167.

[40] J. D. Edelstein, A. Paredes and A. V. Ramallo, "Let's twist again: General metrics of G(2) holonomy from gauged supergravity," JHEP 0301 (2003) 011, hep-th/0211203.

J. D. Edelstein, A. Paredes and A. V. Ramallo, "Singularity resolution in gauged supergravity and conifold unification," Phys. Lett. B 554 (2003) 197, hep-th/0212139.

[41] R. Hernandez and K. Sfetsos, "An eight-dimensional approach to G(2) manifolds," Phys. Lett. B 536 (2002) 294, hep-th/0202135.

R. Hernandez and K. Sfetsos, "Holonomy from wrapped branes," Class. Quant. Grav. 20, (2003) S501, hep-th/0211130.

[42] K. Behrndt, "Singular 7-manifolds with G(2) holonomy and intersecting 6-branes," Nucl. Phys. B 635 (2002) 158, hep-th/0204061.

[43] C. I. Lazaroiu and L. Anguelova, "M-theory compactifications on certain 'toric' cones of G(2) holonomy," JHEP 0301 (2003) 066, hep-th/0204249.

L. Anguelova and C. I. Lazaroiu, "M-theory on 'toric' G(2) cones and its type II reduction," JHEP 0210 (2002) 038, hep-th/0205070.

[44] G. Papadopoulos and P. K. Townsend, "Compactification of $D=11$ supergravity on spaces of exceptional holonomy," Phys. Lett. B 357, 300 (1995) [arXiv:hepth/9506150].

[45] C. Vafa and E. Witten, "A One loop test of string duality," Nucl. Phys. B 447, 261 (1995), hep-th/9505053.

[46] M. J. Duff, J. T. Liu and R. Minasian, "Eleven-dimensional origin of string / string duality: A one-loop test," Nucl. Phys. B 452, 261 (1995), hep-th/9506126.

[47] E. Witten, "On Flux Quantisation in M-Theory and the Effective Action", J. Geom. Phys. 22, 1 (1997), hep-th/9609122.

[48] S. Gukov, C. Vafa, E. Witten, "CFT's From Calabi-Yau Four-folds", Nucl.Phys. B584 69 (2000), [Erratum-ibid. B608, 477 (2001)], hep-th/9906070.

[49] S. Gukov, "Solitons, Superpotentials and Calibrations", hep-th/9911011.

[50] B. Acharya, X. de la Ossa and S. Gukov, "G-flux, Supersymmetry and Spin(7) manifolds", hep-th/0201227.

[51] M. Becker and D. Constantin, "A note on flux induced superpotentials in string theory," JHEP 0308 (2003) 015, hep-th/0210131.

[52] M. Becker, D. Constantin, S. J. J. Gates, W. D. . Linch, W. Merrell and J. Phillips, "M-theory on $\operatorname{Spin}(7)$ manifolds, fluxes and 3D, N = 1 supergravity," Nucl. Phys. B683 (2004) 67, hep-th/0312040.

[53] Y.-H. He, "G2 Quivers," hep-th/0210127.

[54] L. J. Dixon, J. A. Harvey, C. Vafa and E. Witten, "Strings On Orbifolds. 1 and 2," Nucl. Phys. B 261 (1985) 678; Nucl. Phys. B 274 (1986) 285. 
[55] S. Shatashvili, C. Vafa, "Superstrings and Manifolds of Exceptional Holonomy," hepth/9407025.

[56] T. Eguchi and Y. Sugawara, "CFT description of string theory compactified on noncompact manifolds with G(2) holonomy," Phys. Lett. B 519, 149 (2001) [arXiv:hepth/0108091].

[57] K. Sugiyama and S. Yamaguchi, "Cascade of special holonomy manifolds and heterotic string theory," Nucl. Phys. B 622, 3 (2002) [arXiv:hep-th/0108219].

[58] R. Blumenhagen and V. Braun, "Superconformal field theories for compact G(2) manifolds," JHEP 0112, 006 (2001) [arXiv:hep-th/0110232].

[59] T. Eguchi and Y. Sugawara, "String theory on G(2) manifolds based on Gepner construction," Nucl. Phys. B 630, 132 (2002) [arXiv:hep-th/0111012].

[60] R. Roiban, C. Romelsberger and J. Walcher, "Discrete torsion in singular G(2)manifolds and real LG," arXiv:hep-th/0203272.

[61] R. Blumenhagen and V. Braun, "Superconformal field theories for compact manifolds with Spin(7) holonomy," JHEP 0112, 013 (2001) [arXiv:hep-th/0111048].

[62] K. S. Narain, "New Heterotic String Theories In Uncompactified Dimensions i 10," Phys. Lett. B 169, 41 (1986).

[63] P. Kronheimer, "Construction of ALE-spaces as hyper-Kahler Quotients," J. Diff. Geom. 28 (1989) 665.

[64] M. R. Douglas and G. W. Moore, "D-branes, Quivers, and ALE Instantons," arXiv:hep-th/9603167.

[65] P.K. Townsend, "The eleven-dimensional supermembrane revisited," Phys.Lett. B350 (1995) 184.

[66] E. Witten, "String theory dynamics in various dimensions," Nucl. Phys. B 443 (1995) 85, [arXiv:hep-th/9503124].

[67] T. Eguchi and A. J. Hanson, "Asymptotically Flat Selfdual Solutions To Euclidean Gravity," Phys. Lett. B 74 (1978) 249; T. Eguchi, P. B. Gilkey and A. J. Hanson, "Gravitation, Gauge Theories And Differential Geometry," Phys. Rept. 66 (1980) 213.

[68] D. Montgomery, L. Zippin, "Topological transformation groups," New York: Interscience, 1955.

[69] J. Levine, "Semi-Free Circle Actions on Spheres," Invent. Math 22 (1973) 161.

[70] J. Gomis, "D-Branes, Holonomy and M-Theory", Nucl. Phys. B606 (2001) 3, hep-th/0103115.

[71] D. Joyce, "Special Lagrangian m-folds in $C^{m}$ with symmetries," math.DG/0008021.

[72] P. Berglund and A. Brandhuber, "Matter from G(2) manifolds," hep-th/0205184.

[73] B.S. Acharya, F. Denef, C. Hofman, N. Lambert, "Freund-Rubin Revisited," hepth/0308046. 
[74] M. Cvetic, G. Shiu and A. M. Uranga, "Chiral four-dimensional $N=1$ supersymmetric type IIA orientifolds from intersecting D6-branes," Nucl. Phys. B 615, 3 (2001) [arXiv:hep-th/0107166].

[75] S. Katz and C. Vafa, "Matter from geometry," Nucl. Phys. B 497, 146 (1997) [arXiv:hep-th/9606086].

[76] A. Strominger, S.-T. Yau, and E. Zaslow, "Mirror Symmetry Is T Duality," hepth/9606040, Nucl. Phys. B479 (1996) 243.

[77] P.S. Aspinwall, B.R. Greene, D.R. Morrison, "Spacetime Topology Change: The Physics of Calabi-Yau Moduli Space," hep-th/9311186.

[78] H. Partouche and B. Pioline, "Rolling among G(2) vacua," JHEP 0103 (2001) 005, [arXiv:hep-th/0011130].

[79] A. Lukas, S. Morris, "Rolling G(2) Moduli," hep-th/0308195.

[80] B.S. Acharya, "On Mirror Symmetry for Manifolds of Exceptional Holonomy," Nucl.Phys. B524 (1998) 269; "Exceptional Mirror Symmetry," in Winter School on Mirror Symmetry, Vector Bundles and Lagrangian Submanifolds, C. Vafa and S.-T. Yau, eds., AMS and International Press, Boston, 2001.

[81] J.-H. Lee and N. C. Leung, "Geometric Structures on $G_{2}$ and Spin(7)-Manifolds," math.DG/0202045.

[82] P. Aspinwall, B. Greene and D. Morrison, "Multiple Mirror Manifolds and Topology Change in String Theory", Phys. lett. B303 (1993) 249, hep-th/9301043.

[83] E. Witten, "Phases of $N=2$ Models in Two Dimensions", Nucl. Phys. B403 (1993) 159, hep-th/9301042.

[84] A. Strominger, "Massless black holes and conifolds in string theory" Nucl. Phys. B 451, 96 (1995), hep-th/9504090.

[85] B. Greene, D. Morrison and A. Strominger, "Black Hole Condensation and the unification of String Vacua", Nucl. Phys. B451 (1995) 109, hep-th/9504145.

[86] I. Klebanov and E. Witten, "Superconformal field theory on threebranes at a Calabi-Yau singularity", Nucl. Phys. B536, 199 (1998), hep-th/9807080.

[87] I. R. Klebanov and M. J. Strassler, "Supergravity and a confining gauge theory: Duality cascades and chiSB-resolution of naked singularities", JHEP 0008, 052 (2000), hepth/0007191.

[88] S. Gukov and D. Tong, "D-brane probes of G(2) holonomy manifolds", hep-th/0202125. S. Gukov and D. Tong, "D-brane probes of special holonomy manifolds, and dynamics of $\mathcal{N}=1$ three-dimensional gauge theories", JHEP 0204, 050 (2002), hep-th/0202126.

[89] A. Loewy, Y. Oz, "Branes in Special Holonomy Backgrounds", hep-th/0203092.

[90] U. Gursoy, C. Nunez, M. Schvellinger, "RG flows from Spin(7), CY 4-fold and HK manifolds to AdS, Penrose limits and pp waves", hep-th/0203124.

[91] C. Vafa, "Superstrings and topological strings at large N", J. Math. Phys. 42 (2001) 2798 , hep-th/0008142. 
[92] K. Dasgupta, K. Oh and R. Tatar, "Geometric transition, large $N$ dualities and MQCD dynamics," Nucl. Phys. B 610 (2001) 331, hep-th/0105066.

K. Dasgupta, K. Oh and R. Tatar, "Open/closed string dualities and Seiberg duality from geometric transitions in $M$ theory," hep-th/0106040.

[93] F. Cachazo, B. Fiol, K. A. Intriligator, S. Katz and C. Vafa, "A geometric unification of dualities," Nucl. Phys. B 628, 3 (2002), [arXiv:hep-th/0110028].

[94] K. Dasgupta, K. h. Oh, J. Park and R. Tatar, "Geometric transition versus cascading solution," JHEP 0201, 031 (2002), [arXiv:hep-th/0110050].

[95] A. Giveon, A. Kehagias and H. Partouche, "Geometric transitions, brane dynamics and gauge theories," JHEP 0112, 021 (2001), [arXiv:hep-th/0110115].

[96] M. Aganagic and C. Vafa, "G(2) manifolds, mirror symmetry and geometric engineering," arXiv:hep-th/0110171.

[97] S. Frolov and A. A. Tseytlin, "R**4 corrections to conifolds and G(2) holonomy metrics," Nucl. Phys. B 632, 69 (2002), [arXiv:hep-th/0111128].

[98] G. Curio, B. Kors and D. Lust, "Fluxes and branes in type II vacua and $M$ theory geometry with G(2) and Spin(7) holonomy," arXiv:hep-th/0111165.

[99] K. h. Oh and R. Tatar, "Duality and confinement in $\mathrm{N}=1$ supersymmetric theories from geometric transitions," arXiv:hep-th/0112040.

[100] H. Ita, Y. Oz and T. Sakai, "Comments on M theory dynamics on G(2) holonomy manifolds," JHEP 0204, 001 (2002), [arXiv:hep-th/0203052].

[101] T. Friedmann, "On the quantum moduli space of M theory compactifications," Nucl. Phys. B 635, 214 (2002), [arXiv:hep-th/0203256].

[102] H. Ooguri and C. Vafa, "Worldsheet derivation of a large N duality," arXiv:hepth/0205297.

[103] H. Fuji and Y. Ookouchi, "Confining phase superpotentials for SO/Sp gauge theories via geometric transition," arXiv:hep-th/0205301.

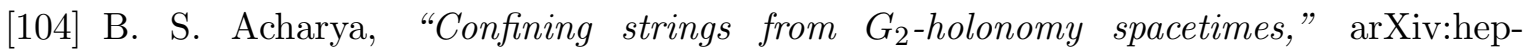
th/0101206.

[105] U. Gursoy, S. Hartnoll, R. Portugues, "The chiral anomaly from M theory," hepth/0311088.

[106] N. Seiberg and E. Witten, "Gauge dynamics and compactification to three dimensions," arXiv:hep-th/9607163. N. M. Davies, T. J. Hollowood and V. V. Khoze, "Monopoles, affine algebras and the gluino condensate," arXiv:hep-th/0006011.

[107] J. A. Harvey and G. W. Moore, "Superpotentials and membrane instantons," arXiv:hep-th/9907026.

[108] M. Bershadsky, C. Vafa and V. Sadov, "D-Branes and Topological Field Theories," Nucl. Phys. B 463, 420 (1996) [arXiv:hep-th/9511222].

[109] D. E. Diaconescu, M. R. Douglas and J. Gomis, "Fractional branes and wrapped branes,” JHEP 9802, 013 (1998) [arXiv:hep-th/9712230]. 
[110] G. Curio, "Superpotentials for M-theory on a G(2) holonomy manifold and trialitysymmetry," JHEP 0303 (2003) 024, hep-th/0212211. 